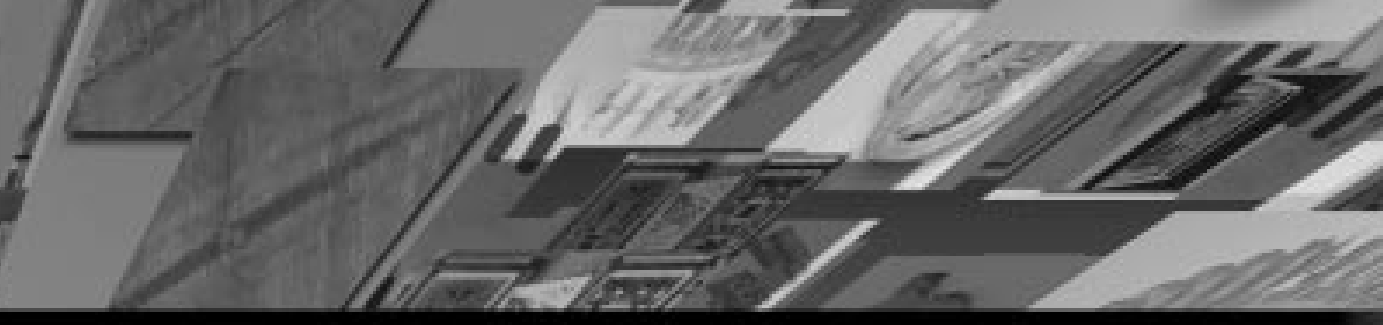

THRUST AREA REPORT • UCRL-ID-125476

\title{
Nondestructive Evaluation
}

\section{Harry E. Martz, Thrust Area Leader}

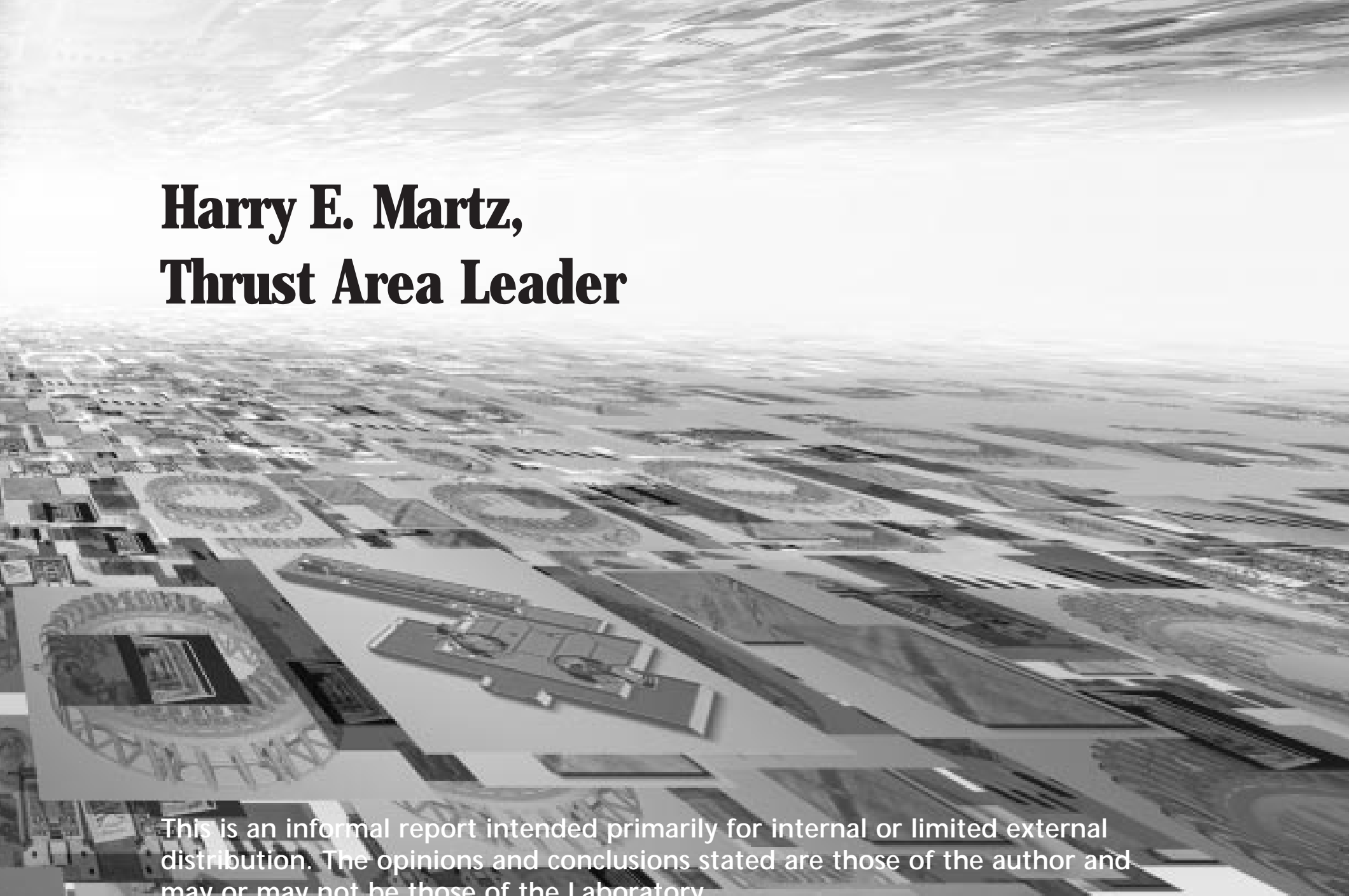

13. Ch is an info mal report intended primarily for internal or limited external alst ibution The opinions and conclusions stated are those of the author and may or may not be those of the Laboratory.

Work performed under the auspices of the U.S. Department of Energy by Lawrence Livermore National Laboratory under Contract W-7405-Eng-48.
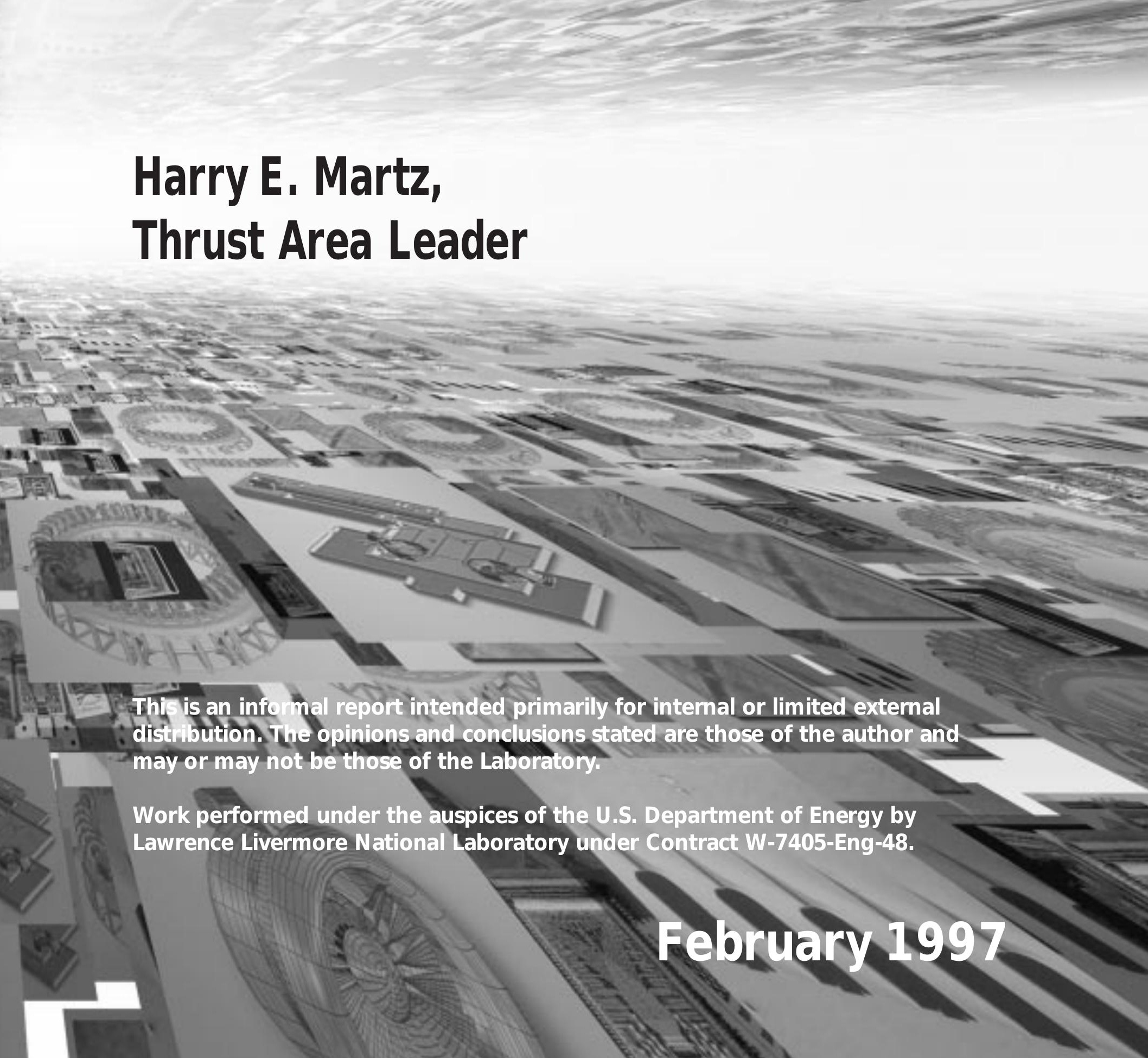


\section{Disclaimer}

This document was prepared as an account of work sponsored by an agency of the United States Government. Neither the United States Government nor the University of California nor any of their employees, makes any warranty, express or implied, or assumes any legal liability or responsibility for the accuracy, completeness, or usefulness of any information, apparatus, product, or process disclosed, or represents that its use would not infringe privately owned rights. Reference herein to any specific commercial products, process, or service by trade name, trademark, manufacturer, or otherwise does not necessarily constitute or imply its endorsement, recommendation, or favoring by the United States Government or the University of California. The views and opinions of authors expressed herein do not necessarily state or reflect those of the United States Government or the University of California, and shall not be used for advertising or product endorsement purposes.

This report has been reproduced

directly from the best available copy.

Available to DOE and DOE contractors from the

Office of Scientific and Technical Information

P.O. Box 62, Oak Ridge, TN 37831

Prices available from (615) 576-8401, FTS 626-8401

Available to the public from the

National Technical Information Service

U.S. Department of Commerce

5285 Port Royal Rd.,

Springfield, VA 22161 


\section{Nondestructive Evaluation}

\section{Harry E. Martz, \\ Thrust Area Leader}

Reprinted from Engineering Research, Development and Technology FY 96 UCRL 53868-96 


\section{Harry E. Martz, Thrust Area Leader}

The Nondestructive Evaluation (NDE) thrust area at Lawrence Livermore National Laboratory (LLNL) supports initiatives that advance inspection science and technology. Our goal is to provide cutting-edge technologies, that show promise for quantitative inspection and characterization tools two to three years in the future.

The NDE thrust area supports a multidisciplinary team, consisting of mechanical and electronics engineers, physicists, computer scientists, chemists, technicians, and radiographers. These team members include personnel that cross departments within LLNL, and some from academia and industry, within the United States and abroad. This collaboration brings together the necessary and diverse disciplines to provide the key scientific and technological advancements required to meet LLNL programmatic and industrial NDE challenges.

NDE provides materials characterization inspections of finished parts and complex objects, to find flaws and fabrication defects and to determine their physical and chemical characteristics. In addition, applying NDE throughout the life cycle of a part saves time and money and improves quality. NDE is being applied to new materials and to process design and development.
NDE also encompasses process monitoring and control sensors and the monitoring of in-service damage. Therefore, NDE is becoming a front-line technology that strongly impacts issues of certification, life prediction, and life extension.

To meet today's programmatic demands, it is important to increase collaboration among LLNL engineering thrust areas and departments. This year we have collaborated with the Power Conversion Technologies and the Information Engineering thrust areas. We have also worked with the Institute for Scientific Computing Research. Such collaborations enable us to stay at the leading edge of NDE technology, research and development.

The primary contributions of the NDE thrust area in FY-96 are described in the six reports that follow: (1) Advanced 3-D Imaging Technologies; (2) New Techniques in Laser Ultrasonic Testing; (3) Infrared Computed Tomography For Thermal NDE of Materials, Structures, Sources, and Processes; (4) Automated Defect Detection for Large Laser Optics; (5) Multistatic Micropower Impulse Radar Imaging for Nondestructive Evaluation; and (6) Multimodal NDE for AVLIS Pod Shielding Components. 


\section{Nondestructive Evaluation}

11 a. 12

1. : : IIII)

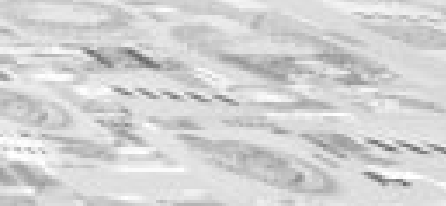




\section{Nondestructive Evaluation}

\section{Overview}

Harry E. Martz, Thrust Area Leader

Advanced 3-D Imaging Technologies

Erik M. J ohansson and Pierre-Louis Bossart.

New Techniques in Laser Ultrasonic Testing

Diane J. Chinn, Robert D. Huber, David D. Scott, Graham H. Thomas, J ames V. Candy,

and J ames Spicer

Infrared Computed Tomography For Thermal NDE Of Materials, Structures, Sources, and Processes

Nancy K. Del Grande, Philip F. Durbin, Dwight E. Perkins, Paul C. Schaich,

Dennis M. Goodman, David H. Chambers, and Thomas Milner

Automated Defect Detection for Large Laser Optics

Laura N. Mascio, Clinton M. Logan, and Harry E. Martz....

Multistatic Micropower Impulse Radar Imaging for Nondestructive Evaluation

J effrey E. Mast, Brooks J ohnston, and Stephen G. Azevedo

Multi-modal NDE for AVLIS Pod Shielding Components

Diane J. Chinn, Nancy K. Del Grande, and Dwight E. Perkins.... 


\title{
dvanced 3-D Imaging Technologies
}

\author{
Erik M. Johansson \\ Defense Sciences Engineering Division \\ Electronics Engineering \\ Pierre-Louis Bossart \\ Manufacturing and Materials Engineering Division \\ Mechanical Engineering
}

We have developed a new software approach to the analysis and visualization of images and volumes. The efficiency of the visualization process is improved by letting the user combine small and reusable applications by means of a machine-independent interpreted language such as Tcl/Tk. These hypertools can communicate with each other over a network, which has a direct impact on the design of graphical interfaces. We have implemented a flexible gray-scale image widget that can handle large data sets, provides complete control of the color palette and allows for manual and semi-interactive segmentation. This visualization tool can be embedded in a data-flow image processing environment to assess the quality of acquisition, pre-processing and filtering of raw data. Our approach combines the simplicity of visual programming with the power of a high-level interpreted language. This report presents applications from biomedical imaging and industrial imaging as examples.

\section{Introduction}

Visualizing images or volumes helps extract qualitative information and quantitative measurements from raw data sets. Visualization software is thus becoming critical in virtually every domain of engineering. However, despite the number of packages available from commercial vendors and from the public domain, it is extremely difficult to find a package that fulfills the needs of a research laboratory dealing with large data sets. Indeed, all the packages are implemented based on the following scheme:

1) First, the file formats need to be decoded, and the raw data read in. The data can then be formatted or extracted. This step includes operations such as sub-sampling, interpolation or dimensional reduction, for example, when a slice is extracted from a 3-D volume.

2) Next, the data are normalized, typically to 8- or 16-bit integers, and displayed in a window after the colors have been allocated. Using predefined color look-up tables, stretching the histogram, reducing the image dynamic range and providing a colorbar help identify the relevant features of the data set.
3) Numerical values, extracted data, or regions of interest can then be written back into a file.

4) At any point, the user may interact with a graphical user interface (GUI) or issue commands to an interpreter, for example, to change the color look-up table or look at pixel values.

Practical experiments showed that none of the command-line oriented (such as VIEW, IDL, PVWave, or Matlab), dataflow-oriented (such as AVS or Explorer) or "self-contained" (such as Analyze or 3DVIEWNIX) visualization packages can handle large data sets whose sizes exceed the sizes of both the memory and the swap space. The user is thus compelled to manually extract smaller pieces of the data sets, which can be time-consuming and inefficient. Therefore, it is mandatory to tightly link the data extraction and data visualization steps, typically by reading and visualizing one slice at a time, instead of loading a 3-D volume in memory before visualizing its 2-D slices.

The efficiency of interactive visualization is also limited by the GUI design, which can almost never be customized by the user. Even when the source code is available, adding or removing features is very 
difficult, if not impossible, since the GUls are implemented as monoliths of hundreds of thousands of lines. However, the user needs to control the way the colors are allocated, for example, interactive thresholds, linear and non-linear color maps. Similarly, extracting profiles, histograms or non-rectangular regions of interest make quantitative measurements possible. Off-line data transforms or extractions are, in our opinion, too cumbersome in a research environment. In addition, unsupervised automatic segmentation performs poorly when the data are noisy. The alternative, that is, the use of manual and semi-automatic segmentation techniques, is limited by the lack of flexibility of most GUls.

The considerations above led us to implement a new visualization tool geared to large data sets. Since we could not afford, nor did we have the experience required to write a self-contained application in X-motif, our approach was to divide the GUI into small reusable components by relying on $\mathrm{Tcl} / \mathrm{Tk}$, which is now used by thousands in every domain of graphical and engineering applications. Tcl/Tk provides simple ways to "glue" different modules together and can be extended easily, in contrast to other GUI builders.

However, the use of $\mathrm{Tcl} / \mathrm{Tk}$ in the signal and image processing community is scarce. Two main reasons can explain this situation. First, efficient data management mechanisms and number-crunching capabilities in image processing are generally believed to require high-performance languages, in contrast to $\mathrm{Tcl}$ which only handles character strings. Next, the photo image widget (a Tcl image display widget) was not designed for interactive visualization, and its flexibility is very limited, mainly because image processing applications are not the main focus of $\mathrm{Tcl} / \mathrm{Tk}$ developers.

In this report, we describe the implementation of a new gray-scale image widget. By focusing on data management and color allocation problems, we were able reach a level of performance which compares favorably with other image processing and visualization packages. A transparent overlay mechanism provides a link to manual and semiinteractive segmentation techniques. We will show how this widget was used to build VISU, a flexible visualization software package described below. VISU is made up of several stand-alone applications which communicate with each other over the network. The power and flexibility of these hypertools in image and volume visualization will be described with some examples. Applications from biomedical imaging and industrial imaging are presented as well.

\section{Progress}

\section{The Pict Gray-scale Image Widget}

As can be guessed from the name, the pict grayscale image widget is based on the photo image widget. After a few experiments, it became clear that the photo widget was not appropriate for our application. First, this widget can only handle 8- or 24-bit color images, and it does not provide any mechanism to visualize floating-point images. Also, the colors are allocated statically and cannot be changed dynamically by the user. Thus, we decided to implement a new widget by focusing on data management and color allocation.

Data Management. Since we wanted to support byte, short, integer or float-point types, we modified the data structure to remove the fields related to color management and dithering. The raw data is allocated in a block of memory and can be accessed by using the data type information. The master structure also provides a pointer to a block of byte data, corresponding to the normalized raw data. The contrast can be increased or decreased by setting the dynamic range of the raw data. This feature proves most helpful when comparing two floating-point images whose dynamic range is different. The pixel values can be queried, and the resultant string contains the actual value, for example a floating-point value.

The Khoros and VIEW file formats are now supported which are local Lawrence Livermore National Laboratory (LLNL) formats. The GIF and PPM readers were modified to read only one color band. Readers/writers for the SUN Raster file format are provided for both the photo and the pict widget. Raw binary files can also be read from a file (or a channel if the code is linked against Tcl7.5) by specifying the dimensions, the data type and the number of bytes corresponding to the header. In all cases, only one slice is read at a time, which dramatically decreases the memory requirements. However, volume rotations and transpositions need to be done off-line. Support will be added in the near future for HDF, netCDF and ACR-NEMA file formats.

Profiling the Photo source code showed that memory management was fairly inefficient, since a lot of time is spent copying blocks between different addresses. The TkPictPutBlock and TkPictPutZoomedBlock routines were rewritten to make sure memory blocks are duplicated only when necessary.

Color Allocation. The human visual system cannot see more than 60 shades of gray, which makes color management for gray-scale images much more simple 
than for color ones. Since we wanted to change the colors dynamically and use pre-defined color maps, we chose to display the images using an 8-bit pseudocolor display. This requirement is, in our opinion, fairly minimal. Besides, our experience proved that dithered images cannot be compared accurately.

To allow for fast array transformations, the colors are allocated from a contiguous set. For example, the palette can be inverted quickly by reversing the color indices. The images are displayed in false colors by choosing from a variety of pre-defined lookup tables. The histogram can be stretched or thresholded to produce a binary image. Furthermore, the colors can be allocated from shared, default or private color maps. Changing the colors of one shared color map will affect all the images that share it. This feature allows the user to visualize the same image displayed with different colors, or to compare the result of two different thresholds.

One additional benefit is that the color allocation can be used to display "semi-transparent" overlays. This is a feature that was found very useful in our interactive segmentation work. The user can, for example, "paint" on the image, draw polygons, Bezier or free-form curves, and yet guess the graylevel values. The overlays can be saved as a mask image. This feature enables the user to extract nonrectangular regions of interest. Alternatively, a mask image can be overlaid on top of the active image to check and verify the accuracy of an off-line segmentation, remove spurious pixels or compare two data sets. The user can interactively combine overlays with logical operations (such as or, xor, and) by changing the Graphic Context. The pict widget provides an interface to advanced image processing routines, ${ }^{1,2}$ and the overlays can also be used in semi-interactive segmentation, ${ }^{3}$ where a coarse initialization is specified. The segmented result can then be displayed.

\section{VISU: A Volume Visualization Application}

In this section, we describe how the pict widget is incorporated in VISU, a user-friendly volume visualization package. In addition to the low-level widget commands, we provide a set of default scripts and high-level commands which make the life of the average user easier. To remove any learning curve for LLNL users, the syntax of these commands reproduces that of VIEW, a general signal processing package used in our group. For example, the following commands will read the first slice of volume "hand", display the image, and then display the tenth slice:
\% rdfile hand 0. sdt $f$

\% disp $f$

\% rdslice f 10

The simplicity of these commands enables the user to write his or her own set of macros. In addition to the command line, VISU also provides a graphical interface to most of the high-level commands. To reduce the GUI design, we chose to provide only one Control Panel and a one-to-one mapping between images and windows. At a given time, one image is considered "active". An image becomes active when the user clicks on it, and its window title is changed. The mouse location and pixel values displayed in the Control Panel correspond to those of the active window. Similarly, moving the slice scale will result in another slice being displayed. The dynamic range of the images can be typed in two entry boxes.

The Palette Menu lets the user change the colors with the mouse. Four scales can be moved to choose the low and high thresholds. The pixels whose values are between the low and high threshold appear white, and the rest appear black. As soon as the scale is changed, the look-up table is updated. Visualizing the actual values of the thresholds instead of normalized values proved very helpful. For example, in our CT applications, the user can see where the attenuation value exceeds a threshold. Pre-defined look-up tables can be loaded by clicking on one of the radio buttons. A color tool makes it possible to stretch the color map in a nonlinear way. The graphical interface allows the user to change the intensity and each of the RGB channels independently to create their own look-up table.

In the Overlays Menu, the active mask can be chosen and overlaid onto the active image. The user can choose how to combine the overlays by setting the overlay Graphic Context with the mouse. For example, the intersection of two binary masks can be seen and saved into a new image. We also provide a graphical interface to our segmentation routines.

The GUI can be customized within minutes without recompiling code. For example, displaying several images side-by-side could be done by packing them in the same canvas, instead of different windows. This flexibility enables us to design the best GUI and to take into account the requirement of a specific imaging application. In most cases, the user will be able to configure the GUI himself.

Releasing the source code on the Internet (ftp://redhook.IInl.gov/pub/visu) helped test VISU on various platforms and operating systems we did not have access to. The configure tool generates Makefiles automatically. While it is still dependent 
on Xlib, VISU can be ported to Windows and Mac-OS without too much effort, to become a machineindependent visualization package. About 500 anonymous ftps were logged on our server. This figure may appear small, but most of the VISU users have very specific needs and would probably not use Tcl/Tk at all if this gray-scale image widget did not exist.

\section{Hypertools}

So far, the features of VISU we described are fairly standard, and can be found in other less flexible visualization packages. However, the Tk library makes it possible to use our widget to build visualization hypertools, defined by Ousterhout ${ }^{4}$ as "stand-alone applications which can communicate with each other and be reused in ways not foreseen by their original designers". Indeed, it came as a surprise to us how easily the send command can be used by visualization tools based on the pict widget in peer-to-peer or master/slave relationships. To demonstrate the power of these hypertools, we discuss several examples.

Image Visualization. We mentioned several times in this report the importance of normalizing floating-point images in the same way. Typically, this can be done by querying the minimum and maximum value of each image, and by computing the absolute minimum and maximum of all the images being displayed. Setting the dynamic range has to be done each time an image is updated; for example, in volume visualization, the dynamic range is likely to be different for each slice. Moreover, visualization tools may run on different systems and display the images on the same screen. In summary, choosing a correct dynamic range is a tedious time-consuming task. However, the send command makes things simple, as the following script demonstrates:

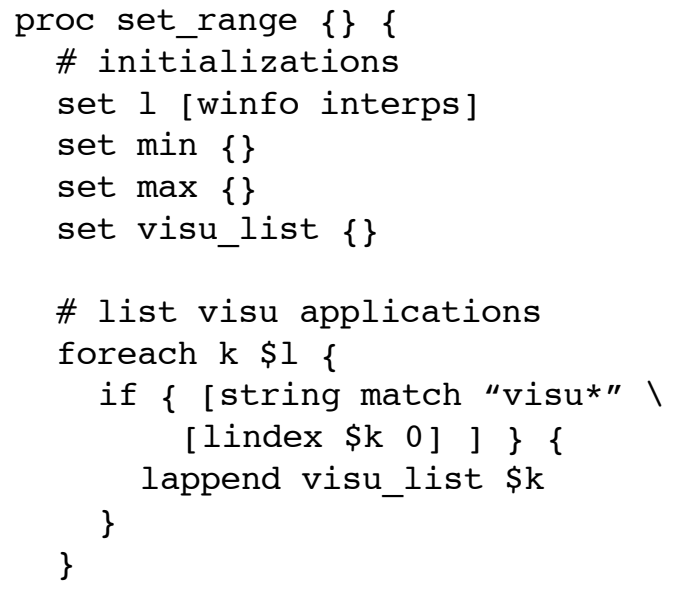

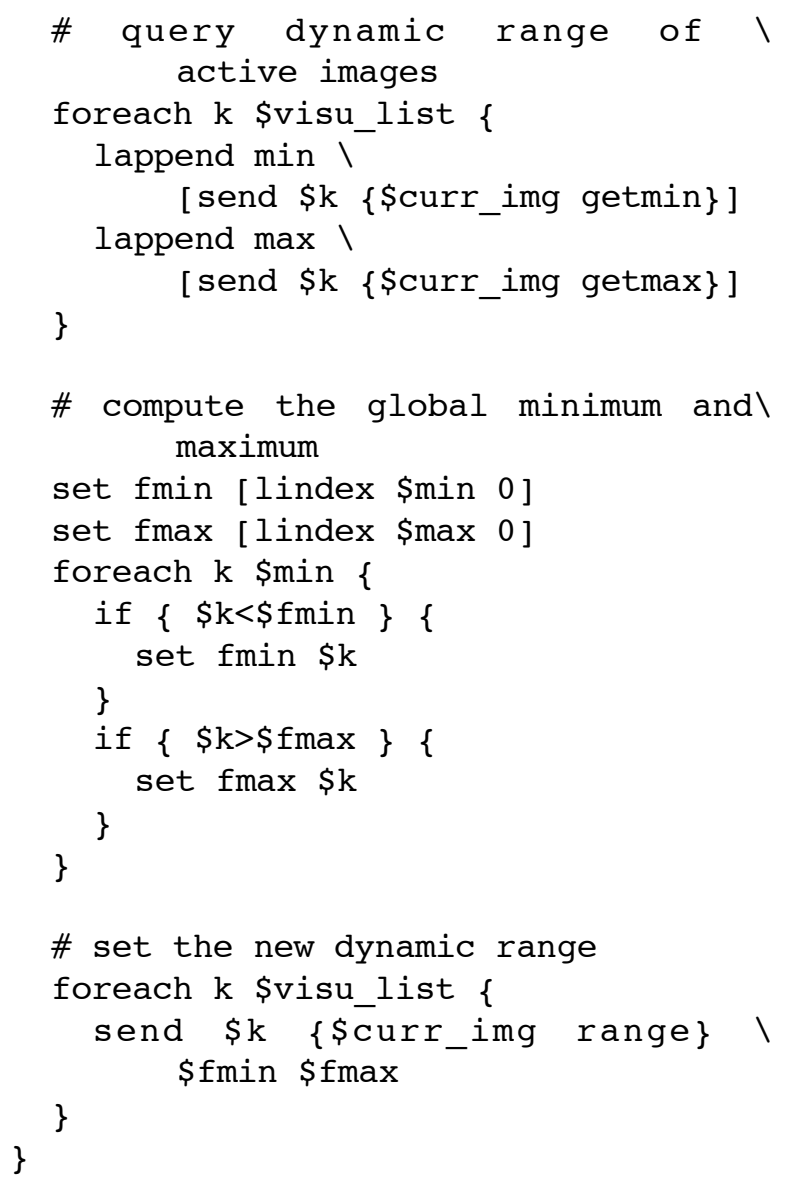

In this script, the dynamic range is broadcast to all the VISU applications. Other possibilities include showing the pixel values at the same mouse location in different images, so as to compare images on a pixel-by-pixel basis. Using the VISU scripts, this option could be written as the following command:

foreach $\mathrm{k}$ \$visu_list \{

send $\$ \mathrm{k}$ \{set $\mathrm{x} \$ \mathrm{x}\}$

send $\$ \mathrm{k}$ \{set $\mathrm{y} \$ \mathrm{y}\}$

send $\$ \mathrm{k}$ set pix । [\$curr_img get $\$ \mathrm{x} \$ \mathrm{y}]\}$

\}

Instead of sending values, it is possible to send commands, and, for example, to visualize the same slice in different data sets by broadcasting the command rdslice \$curr_img \$curr_slice. A direct application is the creation of simultaneous animations of volumes located in different file systems and accessed over the network.

Image Profiles and Histograms. Visualization of image profiles along an arbitrary line is an invaluable tool to evaluate the presence of artifacts in reconstructed images. For example, in $\mathrm{x}$-ray $\mathrm{CT}$, beam-hardening produces "cupping" artifacts that can easily be detected by extracting a profile. 
Similarly, the accuracy of the reconstruction techniques can be assessed by visualizing the profiles of sharp edges. Typically, low-pass filtering and noise elimination smooth and spread the transitions.

Along the same lines, visualizing a histogram helps understand the statistics of an image and the distribution of its gray levels. In the case where the histogram is made up of several modes, the objects can typically be segmented out by applying different thresholdings to the data.

Although most image visualization packages provide 1-D signal viewers, our experience proved that they cannot be easily customized by the user and are not flexible enough.

In our applications, the 1-D signals are extracted by issuing a command to the pict image widget and sent to a BLT graph. Tcl/Tk handles only character strings and relies on the $X$ protocol to communicate between applications. In addition, the profiles are generally made of less than 1000 samples, so that the overhead introduced by the communications is minimal. This feature allows the user to configure the graphical interface. The main benefit of this approach is that the user can dynamically create and extract new profiles or histograms of images viewed across the network, combine and compare them without having to save these 1-D signals in files, and transfer them by ftp.

Data-flow Environments. In our biomedical application, all the data pre-processing and segmentation is implemented in Khoros, a data-flow image processing environment. Khoros provides a network editor. The input data undergo a series of transformations each time a network node is executed by the scheduler. This data flow environment helps the user design an imaging application, as the output of each node can be visualized by connecting it to a visualization module. (Other packages such as AVS, Explorer, LabView use the same paradigm.) However, dynamically setting the range of all the images in a data flow environment is next to impossible. Similarly, writing a macro or a loop in a command-line interface sometimes makes more sense than editing a network of nodes.

These two problems were solved by implementing a Khoros module that executes a VISU script. This approach results in several important benefits. First, it compensates for some of the drawbacks of VISU, for example by providing simple ways to extract arbitrary slices. Basically, we rely on modules provided in the Khoros distribution, or write our own modules to filter and transform the raw data.

Next, the power of both command-line and dataflow environment can be combined. A command-line interface is provided by a remote controller derived from the rmt example of the Tk distribution, which can communicate either with a specific application or send messages to all of them. As a result, the user can rely on the power of visual programming, while being able to rely on a more traditional commandline interface. To our knowledge, this feature is not provided by any other visualization package.

Finally, the embedded visualization modules can communicate with each other. For example, the same profile could be extracted in different images and displayed in the same 1-D signal viewer. The same approach could be easily implemented in other data-flow environments such as AVS or Explorer.

Volume Visualization. As described before, VISU can display slices of a 3-D volume. Another way to visualize a volume is to extract iso-surfaces, either by using the 3-D Marching Cubes algorithm ${ }^{5}$ or by reconstruction of a 3-D surface from 2-D contours. ${ }^{6}$ Visualizing a surface helps understand the spatial distribution of objects in 3-space, in contrast to visualizing 2-D slices. Almost every visualization package provides these tools, but they are almost never combined or used simultaneously.

Several other approaches make use of $\mathrm{Tcl} / \mathrm{Tk}$ in volume visualization. Schroeder, Lorensen and Martin recently released vtk (http://www. cs.rpi.edu:80/ martink), a visualization tool kit which can be used either by writing $\mathrm{C}++$ programs, or through Tcl/Tk scripts. Similarly, Lacroute ${ }^{7}$ released VolPack (http://www.graphics.stanford. edu/software/volpack/), a volume rendering library coupled with a $\mathrm{Tcl} / \mathrm{Tk}$-based graphical interface.

These two tool kits could be used in conjunction with VISU to build a volume visualization package by relying on the send command. This approach results in two main benefits.

First, the different visualization modules can be combined easily, even if they are not executed on the same machine. Typically, it will be possible to highlight a point of the surface and to see the intersecting slice. Conversely, viewing a new slice will automatically change the coordinates of this highlighted point. The color tools provided with VISU will also be used to choose iso-surface values, or the transfer functions in volume rendering. The same graphical interface can also be used to set the viewing parameters in surface and volume rendering. It is our belief that the combination of these visualization techniques will help identify more efficiently the relevant features of volumetric data sets.

Second, provided that the interface between hypertools does not change, the user does not need to know how the surface or volume rendering techniques are implemented. As a result, the best 
visualization modules can be chosen by the user. For example, vtk supports an abstract rendering engine and can be slower than the tkSM widget (http://www.isr.umd.edu/ ihsu/tksm.html), which provides easy access to the OpenGL and Mesa libraries. In the case where the software modules were not linked against Tcl/Tk, they can still be used and executed from the $\mathrm{Tcl} / \mathrm{Tk}$ environment.

\section{Applications}

We present two examples to illustrate the power and flexibility of VISU: a biomedical imaging application and an industrial imaging application. In the first example, we used VISU to interactively segment CT image data of a human hand, which was later used to generate a 3-D mesh for use in FEM modeling of the bone structure ${ }^{8}$. Figures $\mathbf{1}$ (a)-(d) show two cross-sections of a CT volume displayed with different look-up tables, demonstrating the capability of both private and shared color maps. The concept of transparent overlays is described in Fig. 2 (a)(c). Finally, the use of overlays in semi-automatic segmentation is presented in Fig. $\mathbf{2}$ (d)-(f).

Our industrial imaging application was to use VISU to analyze CT image reconstructions of vesicular basalt. This was a proof-of-principle experiment conducted in cooperation with the Complex Systems Research Center, Institute for the Study of Earth, Oceans, and Space at the University of New Hampshire (UNH). The goal of the analysis was to determine the size distribution of vesicles (voids) in a basalt sample supplied by UNH. Because of the non-spherical nature of the vesicles, the problem was to determine the volume distribution of the vesicles and their volume fraction. Currently, graduate

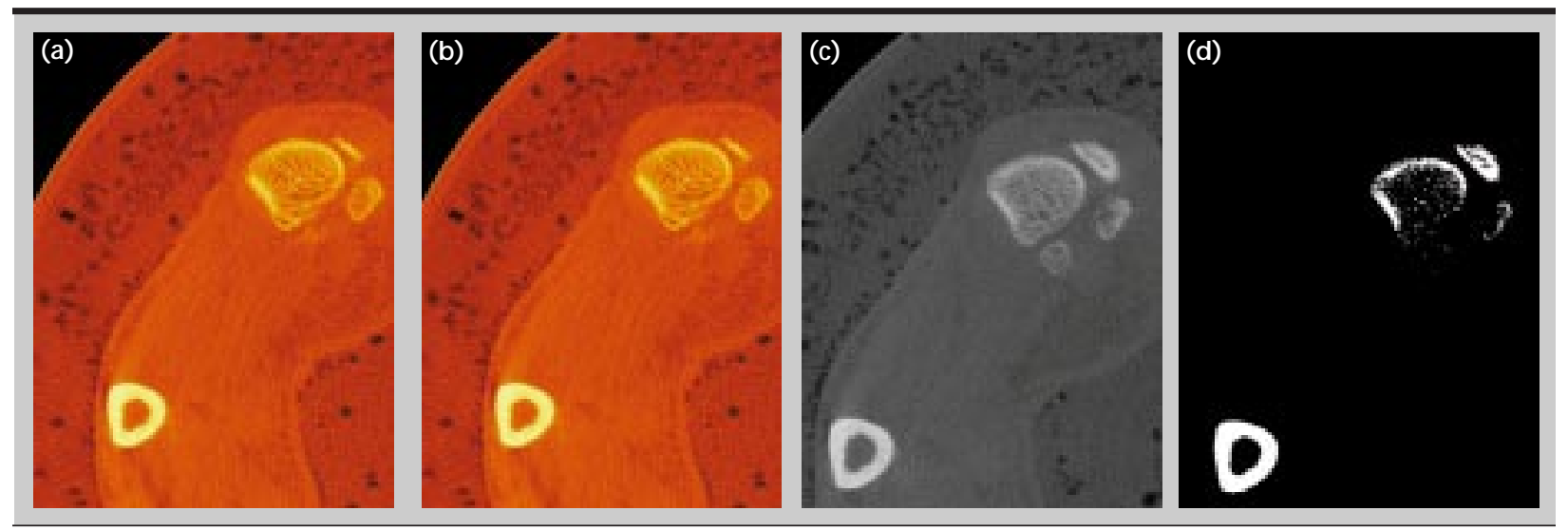

Figure 1: Cross-sections of a thumb and index near joint: (a) corresponds to slice 308 displayed with a 'ct' private color map; (b) is also slice 308 with a shared color map; (c) corresponds to slice 314 with a 'gray' private color map; (d) is slice 314 with a shared color map. In this example, the private color maps are used as a reference while the colors are changed simultaneously in the two shared color maps.

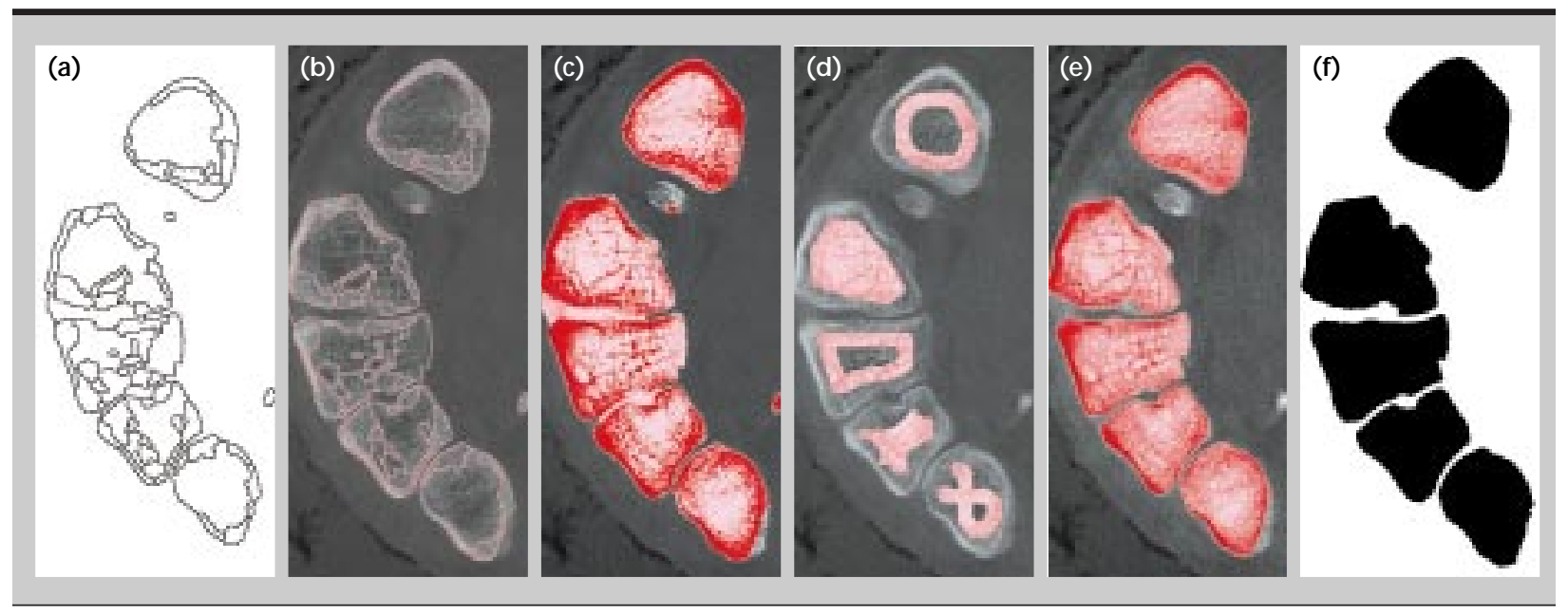

Figure 2: Cross-section of a hand: (a) contours from an off-line segmentation; (b) contours overlaid on the image; (c) regions created from these contours; (d) coarse manual initialization of overlays; (e) interactive segmentation result after region-growing; (f) resulting segmented mask. 
students at UNH compute the volume fraction by (1) destructively slicing through basalt samples, (2) estimating the representative sizes of the voids in the slice using manual measurements, and (3) using the volume of the original sample to determine the volume fraction of the voids. It is a timeconsuming and error-prone process. We wanted to make the process nondestructive using $\mathrm{CT}$, and automate it using the capabilities of VISU.

A photo of the basalt sample is shown in Fig. 3. It was taken from the Drakensburg Basalts of southern

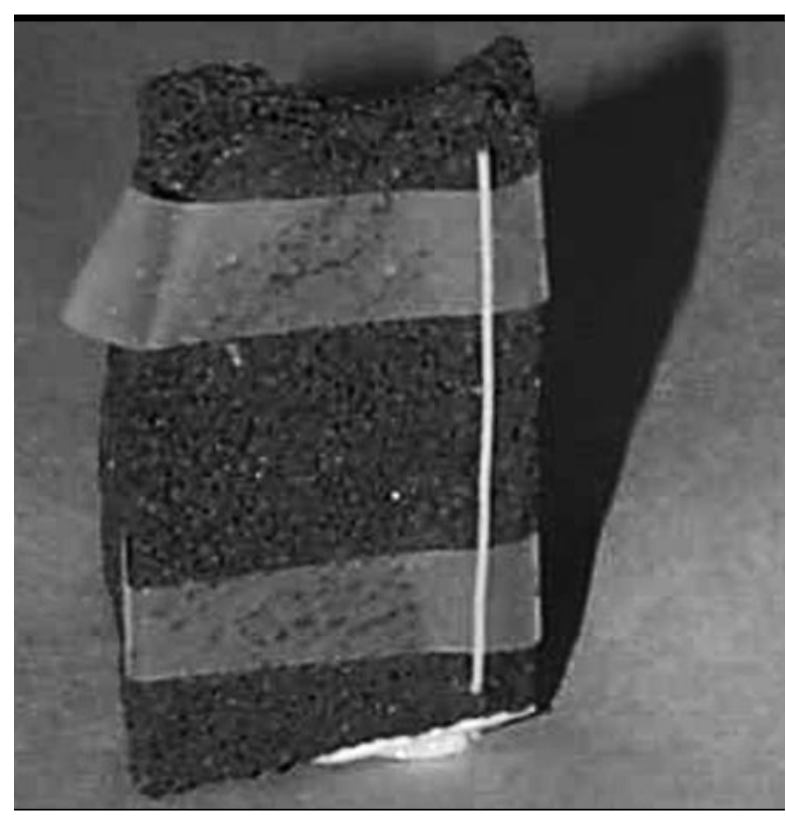

Figure 3: Photograph of the basalt sample used in the industrial imaging application. Note the very porous nature of the sample and the $0.76-\mathrm{mm}$-diameter aluminum fiducial wire taped to it.

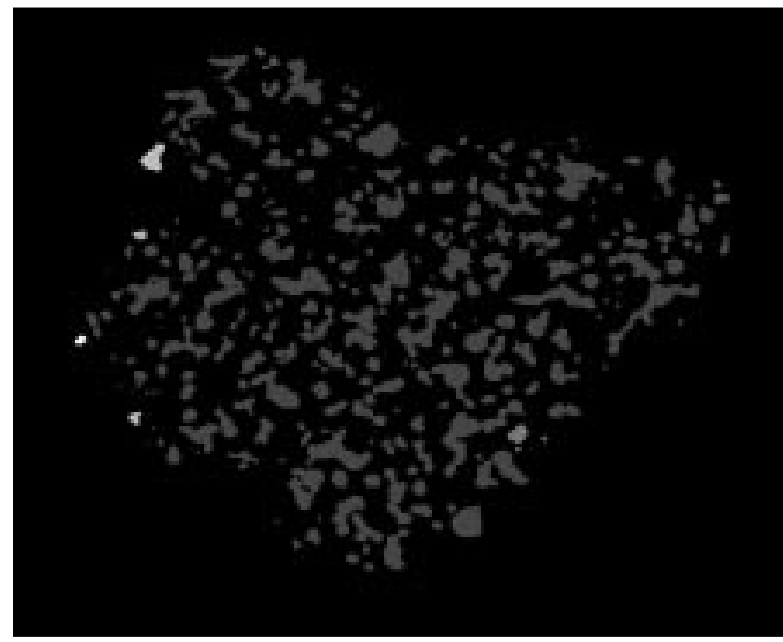

Figure 4: A segmented image slice of the sample representing voids.
Africa. A $0.76-\mathrm{mm} \mathrm{Al}$ wire was taped to the sample for use as a fiducial in the image analysis. CT projection data were recorded using the LLNL PCAT CT scanner. 101 slices were reconstructed at a resolution of $60 \mu \mathrm{m}$. The analysis was performed in VISU by segmenting the image volume into two binary images, one representing voids and one representing basalt. Sample segmented image slices are shown in Fig. 4 (voids) and Fig. $\mathbf{5}$ (basalt). A connected component analysis was performed on the two volumes, showing that the voids are indeed complex structures connected in three dimensions. The volume of the voids was easily computed by summing over the void volume image and scaling by the pixel volume. The process was verified by computing the volume of the Al wire, which was known a priori. The volume fraction of the voids was shown to be $24 \%$. The experiment was successful, showing that VISU can easily be used to accurately estimate the porosity of a volume image.

\section{Future Work}

Although $\mathrm{Tcl} / \mathrm{Tk}$ is widely distributed in engineering and graphical applications, its use in imaging and visualization is recent. In this report, we described a new gray-scale widget and its use in visualization of large data sets. Although it relies on an interpreter, its speed compares favorably with typical X-motif self-contained applications, since we implemented an efficient management of data and colors. Designing high-level commands and combining hypertools helps users perform tedious tasks automatically, thus increasing the interaction efficiency. We plan to enhance our image processing library and to port the

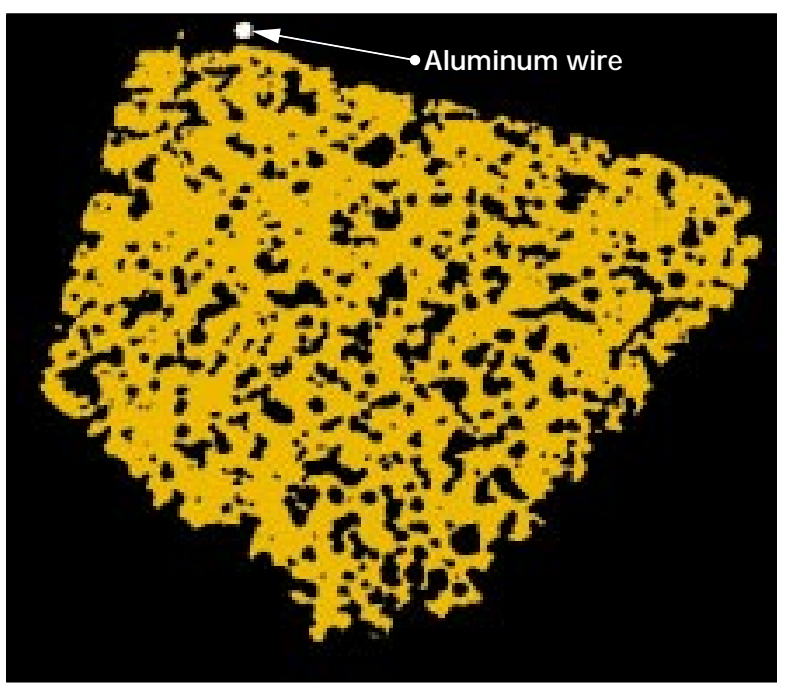

Figure 5: A segmented image slice of the sample representing the basalt. Note the cross-section of the aluminum wire at the top of the image. 
code to Windows and Mac. The Tcl7.5 sockets will also be used to develop distributed visualization applications for the Internet.

\section{Acknowledgments}

We acknowledge the support of $\mathrm{H}$. Martz and K. Hollerbach. J. Pearlman, M. Abramowitz, E. Nicolas, J. P. Hebert, M. Cody and D. Garrett helped improve VISU by providing invaluable bug reports and suggestions.

\section{References}

1. Vincent, L., and P. Soille (1991), "Watersheds in Digital Spaces: An Efficient Algorithm Based on Immersion Simulations," I.E.E.E. Transactions on Pattern Analysis and Machine Intelligence, Vol. 13, (6), J une, pp. 583-598.

2. Vincent, L. (1993), “Morphological Grayscale Reconstruction in Image Analysis: Applications and Efficient Algorithms," I.E.E.E. Transactions on Image Processing, Vol. 2, (2), April, pp. 176-201.
3. Bossart, P-L. (1994), Détection de contours réguliers dans des images bruitées et texturées: association des contours actifs et d'une approche multiéchelle, Thèse, Institut National Polytechnique de Grenoble, France, Octobre.

4. Ousterhout, J. K. (1994), TCl and the Tk toolkit, Addison-Wesley, Reading, Mass., (ISBN 0-20163337-X).

5. Lorensen, W. E., and H. E. Cline (1987), "Marching Cubes: A High Resolution Surface Extraction Algorithm," Computer Graphics, Vol. 21, (3), pp. 163-169.

6. Geiger, B. (1993), Three-Dimensional Modeling of Human Organs and its Application to Diagnosis and Surgical Planning, Ph.D. thesis, Ecole des Mines de Paris, France.

7. Lacroute, P. G. (1995), Fast Volume Rendering Using a Shear-Warp Factorization of the Viewing Transformation, Ph.D. thesis, Stanford University, Stanford, Calif. (CSL-TR-95-678).

8. Hollerbach, K. (1996), "Modeling Human J oints and Prosthetic Implants," LLNL Science and Technology Review, September, (UCRL-52000-96-9) pp. 19-21. 


\title{
ew Techniques in Laser Ultrasonic Testing
}

\author{
Diane J. Chinn, Robert D. Huber, \\ David D. Scott, and Graham H. Thomas \\ Manufacturing and Materials Engineering Division \\ Mechanical Engineering
}

\author{
James V. Candy \\ Imaging and Detection Program \\ Laser Programs
}

James Spicer

Johns Hopkins University

Baltimore, $M d$.

Laser ultrasonic testing is an exciting new area of nondestructive evaluation (NDE). Using modern, up-to-date optical methods for generating and detecting ultrasonic waves in materials, laserbased ultrasonic testing is beginning to find a wide-range of novel applications. Several key disadvantages of conventional ultrasonic testing, a technique that has been in use for the last thirty years, are overcome with laser ultrasonics. Lawrence Livermore National Laboratory (LLNL) brings unique optics and signal processing capabilities to the development of laser ultrasonics and is poised to make major contributions to the field. As applications of NDE to LLNL programs become more demanding, with many different constraints, development of new NDE techniques such as laser ultrasonic testing is required. We are using LLNL technical expertise in collaboration with J ohns Hopkins University to develop several areas of laser ultrasonic testing. In addition, we continue to support LLNL programs by developing laser ultrasonic testing solutions for a diverse set of applications.

\section{Introduction}

Technological advances in materials development, tool design and production methods present new challenges for NDE techniques. Many of these challenges are addressed by developments in laser ultrasonic testing. Conventional ultrasonic testing is commonly used in many applications to characterize material properties, find defects, measure thickness of components, study crack growth and monitor other quality parameters. However, conventional ultrasonic testing is limited to applications where high-frequency acoustic energy can be mechanically transmitted into a test specimen through a couplant. Laser-based ultrasound offers a method of coupling sound into and out of materials through optical generation and detection. Non-contacting optical testing of parts increases the flexibility of ultrasonic testing. Parts in hostile environments, in inaccessible areas, with complex geometries and in rapid production environments can all be evaluated using the noncontacting laser ultrasonic method. The major disadvantage of laser ultrasonic testing is its inherently poor sensitivity when compared to conventional ultrasonic testing.

\section{Progress}

This year we implemented both hardware modifications to our existing system and software signal enhancements to our processing algorithms to increase sensitivity and flexibility. Improvements to our path-stabilization circuitry increase the detection sensitivity level. Development of fiber-optic detection hardware permits remote sensing of ultrasonic wave fields in parts. Assembly of a data acquisition and scanning system allows the rapid collection of large amounts of data from which images can be generated. 
Enhanced imaging and flaw localization algorithms increase our sensitivity by extracting more useful information from our collected data. Finally, our main effort this year focused on developing prototypes for several LLNL applications.

\section{Hardware Modifications}

Our present system uses a Nd:YAG pulsed laser to generate an ultrasonic wave and a $\mathrm{He}: \mathrm{Ne}$ Michelson laser interferometer to detect the propagated wave. The generation laser delivers a $17 \mathrm{~mJ}$ pulse to heat a 3-mm spot on the surface of a part for 4 ns. Figure 1a shows the present configuration of our Michelson interferometric detection system.

Improved Stabilization Circuits. The Michelson interferometer, used to detect surface displacements resulting from the propagation of ultrasonic waves in materials, is a path-stabilized device. Ambient vibrations present in most measurements are considerably larger in amplitude than the bulk ultrasonic waves. Because Michelson interferometers are affected by ambient vibrations, a path-stabilization scheme is used to correct for these vibrations. Frequencies of ambient vibrations are generally much lower than ultrasonic wave frequencies. Path stabilization on the Michelson interferometer takes place through the reference leg and the piezoelectric tube shown in Fig. 1a. The path-stabilization process involves the use of a feedback electronic circuit that drives a piezoelectric tube attached to the reference mirror of the interferometer. A correction circuit powers the piezoelectric tube, driving the reference mirror at frequencies that cancel out the effects of ambient vibrations.

Improvements in electronic devices have permitted us to make modifications to the correction circuit. We designed a new correction circuit that allows more efficient cancellation of the ambient vibrations, thereby improving the operation of the Michelson interferometer. The correction circuit uses state-of-the-art electronic components. The primary new component is the PA88 High Power Operational Amplifier circuit chip which is designed specifically to drive piezoelectric crystals and replaces several transistors. In addition, the piezoelectric tube is replaced by a stack of piezoelectric wafers that is more efficient in correcting for vibrations.

Fiber-Optic Detection. Fiber optics increase the flexibility of optical detection of ultrasonic wave propagation. Fiber-based detection permits large distances between equipment and test specimen, allows accessibility to hostile or hard-to-reach environments and facilitates changes to the test configuration. By permitting large distances between instruments and the material being tested, exposure of sensitive equipment to hostile environments can
Figure 1. Laser ultrasonic detection interferometer, modified to include fiber-based detection: (a) shows the original configuration for the Michelson interferometer; (b) shows optical fiber used for the signal leg of the Michelson interferometer; (c) displays direct beam generation; (d) shows direct beam generation with fiber-optic detection, reducing sensitivity by a factor of 4 , but leaving comparable SNR. (a)

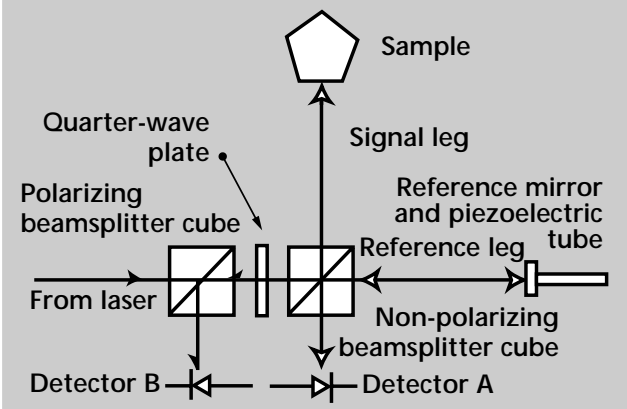

(c)

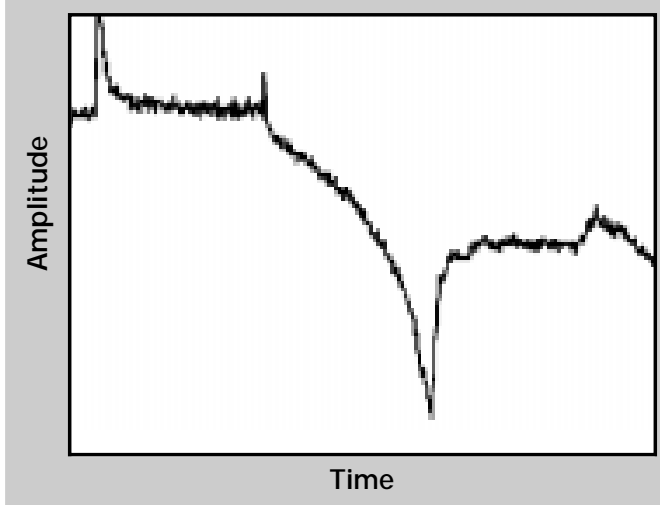

(b)

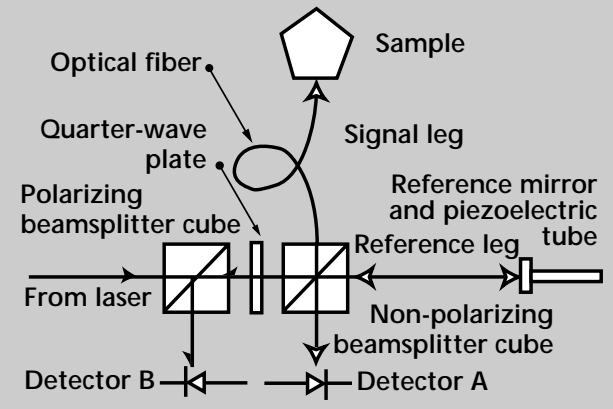

(d)

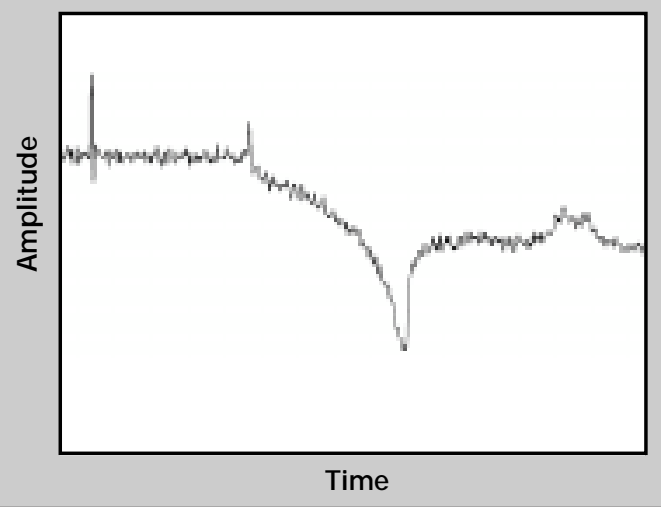


be eliminated. When scanning a part, only the fiber needs to be moved, decreasing the bulk and expense associated with staging equipment. Fibers are especially useful on applications that require testing in a glove box where large, heavy instrumentation may render testing impossible. When using light-weight fiber-optic cables, only the fibers need to be brought to the actual test location, and the electronics can remain in a remote vehicle or laboratory, or at a safe distance from the test surface.

The signal leg of the Michelson interferometer, shown in Fig. $\mathbf{1}$ b, is placed in optical fiber. Figures 1c and $\mathbf{1 d}$ show a comparison of direct beam (non-fiber) laser-generated ultrasonic signals in a polished, 12-mm-thick aluminum plate detected using a direct beam and a fiber-optic in the signal leg, respectively. While the sensitivity of the received signal is reduced by approximately four, the signal-to-noise ratio (SNR) remains about the same because of the smaller surface spot size. These preliminary results appear very promising for the use of fibers in LLNL applications.

\section{Data Acquisition and Scanning System}

High-resolution imaging is important to many applications where defect-sizing and mapping inhomogeneities are required. We have assembled a LabView-based data acquisition and scanning system featuring high-precision gigahertz sampling rates and scanning with micron resolution.

Signal Enhancing Software. We applied the model-based reference processor ${ }^{1,2}$ developed last year to various experimental data sets. The processor enhances noisy measurements and provides SNR improvements. This year we investigated techniques to extend the data from our measurement system by enhancing the noisy data through the improved signal processing capability, creating a "synthetic aperture" to gather both spatial and temporal data, and developing imaging techniques that enable us to analyze the spatio-temporal displacement field in a test specimen. The goal is to use the spatiotemporal characteristics of the specimen displacement wave-field to isolate flaws or scatterers.

\section{Imaging}

To date most laser ultrasonic imaging techniques use a monostatic configuration for laser generation and detection. Both source and detector move together in tandem while the surface displacement signature is recorded. The spatio-temporal approach uses a bistatic configuration to create a synthetic receiving array. In the bistatic configuration, the source remains fixed while displacements at the detector are recorded at multiple locations. This configuration is depicted in Fig. $\mathbf{2 a}$, along with the expected response predicted by our thermoacoustic modeling program WAVER ${ }^{3}$ (Fig. $\mathbf{2} \mathbf{b}$ ). In this manner a complete spatio-temporal signal can be used to reconstruct the displacement wavefield throughout the specimen.

To demonstrate the viability of this approach, we generated a set of modeled data for a $12-\mathrm{mm}$-thick aluminum plate with a compressional wave speed of $6.32 \mathrm{~mm} / \mu \mathrm{s}$ and a shear speed of $3.16 \mathrm{~mm} / \mu \mathrm{s}$. The modeled data predicts the surface displacement response from a 16-element linear array of interferometric detectors, spaced $1 \mathrm{~mm}$ apart, resulting from a single source sending an ultrasonic wave propagating throughout the medium.

We used this data to image the displacement field by developing a spatio-temporal filter, or beamformer. ${ }^{4}$ A beamformer takes an array of spatially discrete responses and temporally filters each element in the array. With this filter, the

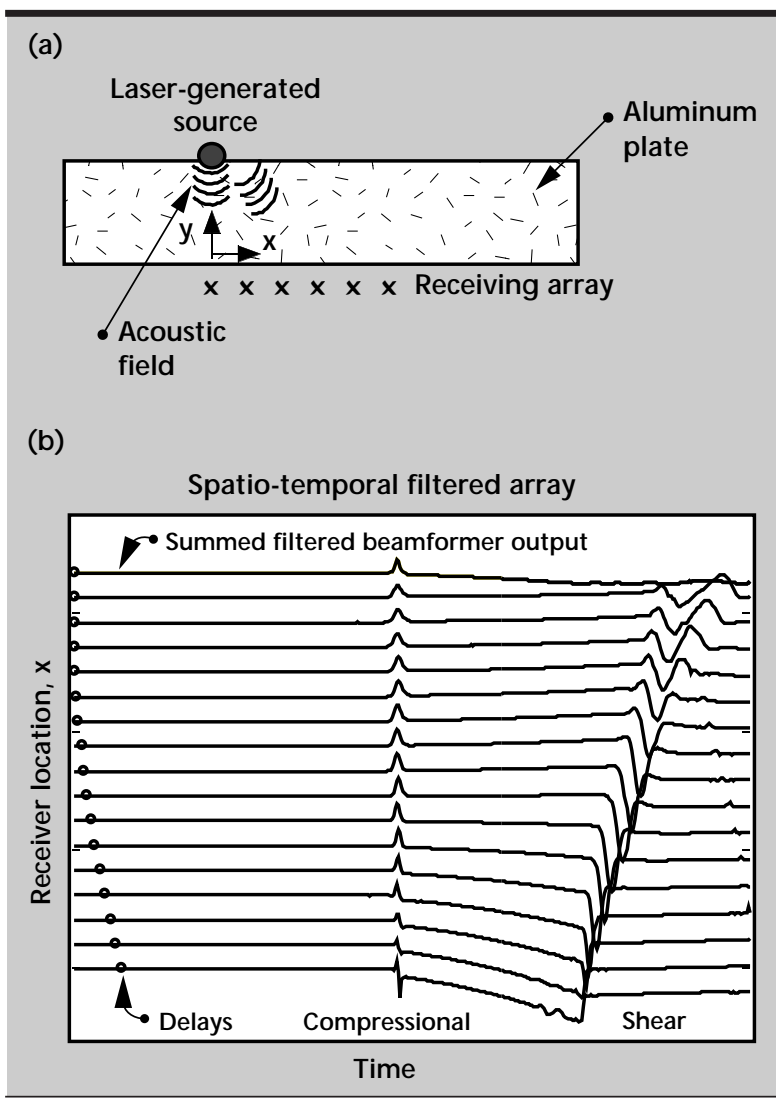

Figure 2. (a) An acoustic field in an aluminum plate, generated from a pulsed laser source. An array of surface displacements on the opposite side of the plate resulting from the thermoelastic source is modeled with the WAVER program. (b) The array of modeled responses, with both compressional and shear wave arrivals. Beamformer output is shown at the top. 
displacement field is assumed to originate from a source at a particular spatial position in the test specimen (aluminum slab) and the appropriate time delays corresponding to this position are calculated. At each individual detector position, or channel, broadband signals are delayed and scaled appropriately. Finally, all of the signals are summed to calculate the location of the reconstructed source.

A typical beamformer output is shown at the top of Fig. $\mathbf{2 b}$. The 16 delayed channel signals, summed to obtain the beamformer signal at the top, show the compressional wave arrival. In this manner compressional displacement fields created by the laser ultrasonic source can be used to generate an image of the specimen. Each pixel in the image has the value of the maximum positive displacement from the beamformer output, derived from the spatial coordinates, channel delays and compressional wave speed, to the pixel position. The results of the modeled displacement field in Fig. $\mathbf{2} \mathbf{a}$ are shown in Figs. $\mathbf{3} \mathbf{a}$ and $\mathbf{3 b}$ for both compressional and shear fields corresponding to peak positive and peak negative values. As expected,
Figure 3. Peak surface displacement images estimated from modeled data, showing the source location within the displacement field resulting from (a) the compressional wavefield (positive peak displacement) and (b) shear wavefield (negative peak displacement). Images generated from measured data show the displacement fields corresponding to (c) the compressional wavefield and (d) the shear wavefield. (a)

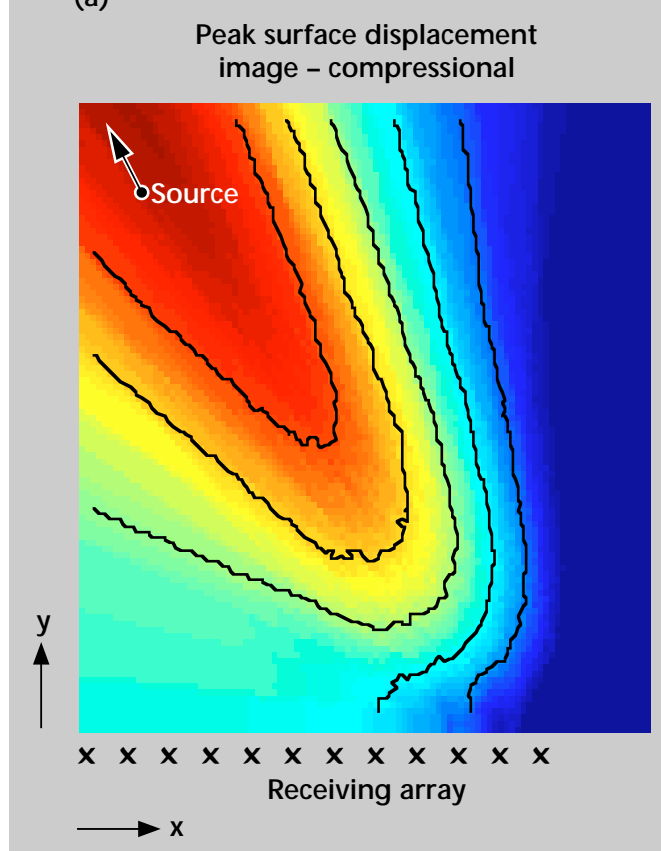

(c)

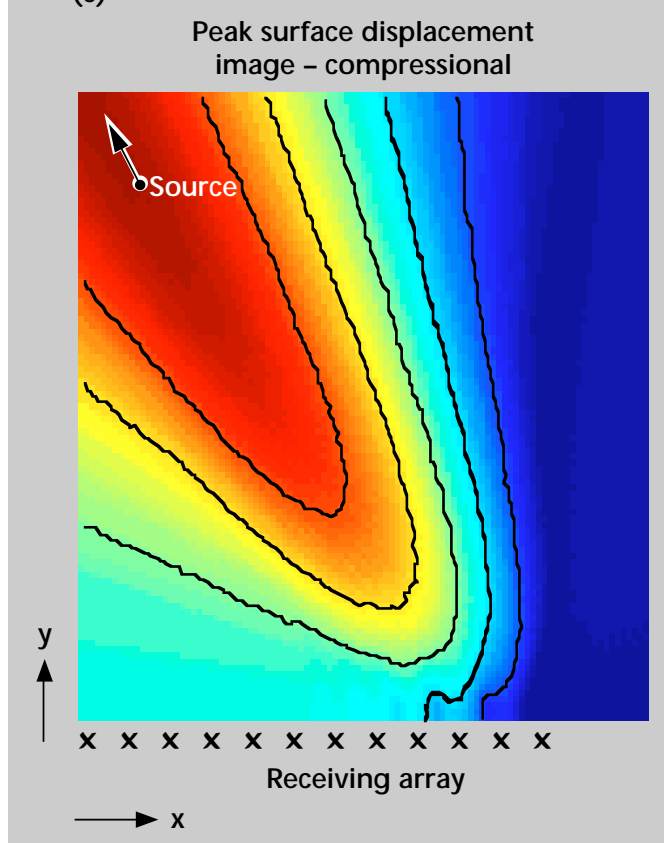

(b)

\section{Minimum surface displacement} image - shear

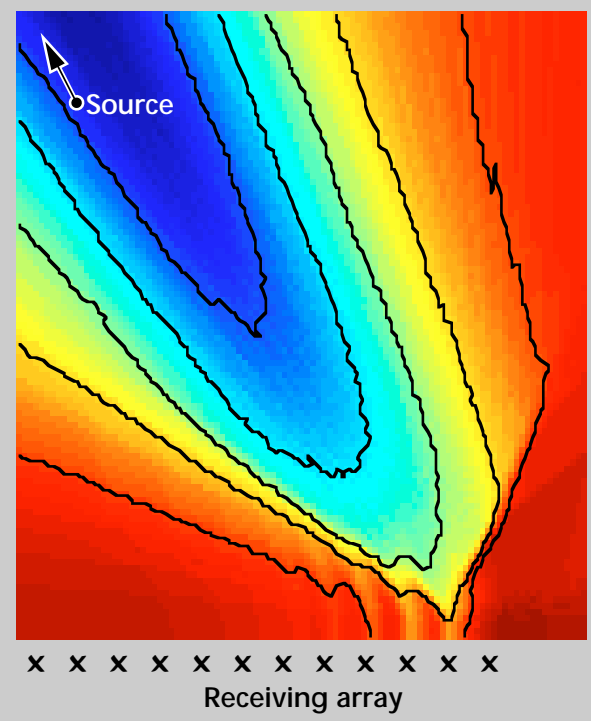

(d)

Minimum surface displacement image - shear

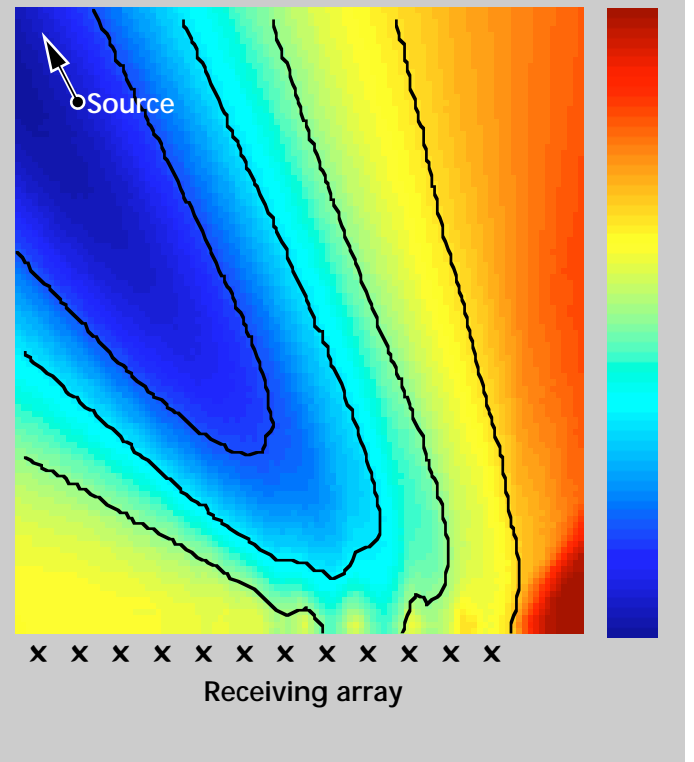


the maximum and minimum displacement values, corresponding to the source of compressional and shear waves, respectively, both occur at the origin ( $x=0 \mathrm{~mm}, y=12 \mathrm{~mm}$ ).

Next, we validate the beamformer performance experimentally for the same material properties and dimensions under the same conditions as those in the simulation. The results are shown in Figs. 3c and $\mathbf{3 d}$. Here we see that the images correspond closely to images in Figs. $\mathbf{3} \mathbf{a}$ and $\mathbf{3} \mathbf{b}$ that were generated from modeled data. The images for simulation and experiment are similar and demonstrate the viability of the beamformer processor.

Flaw Localization with Beamforming. We have also begun development of a flaw detector and localizer based on the beamformer imaging approach. We are extending our numerical simulator to predict scattered responses and developing validation experiments. However, to test the flaw detector we modeled the response from two ideal point scatterers located in a 12-mm aluminum plate, as shown in Fig. 4a. The scatterers are located between the source and the 16-element receiving array used in the previous beamformer configuration. The modeled noise-free spatio-temporal response, assuming scatter by a compressional wave, is shown in Fig. 4b. Figure 5a shows the image derived using the beamformer imaging technique. The point scatterer closest to the detectors (scatterer \#1 in Fig. 4a) is better defined by the beamformer imaging than the point scatterer farther from the detector array (scatterer \#2 in Fig. 4a).

Matched-filter Processing. To improve resolution as well as detection capability, we use information about the source of ultrasonic wave generation. For this modeled case the source function is used to develop an optimal spatio-temporal "matched-filter" processor. By using a multichannel time-reversal (TR) processor, ${ }^{5}$ we filter the data with the timereversed replicant of the scatterer propagated to the array from each assumed scatterer position. In this manner, an image proportional to the square of the surface displacement power is created, shown in $\mathbf{F i g} \mathbf{5 b}$. The matched-filter image in Fig. $\mathbf{5 b}$ resolves scatterer \#2 better than the beamforming image in Fig. 5a. We hope to continue exploring this processor next year to produce specimen images which enable reliable detection and localization.

\section{Applications- Laser Cut Monitoring}

High power, femtosecond lasers can be used to cut materials with minimal kerf and insignificant collateral damage. This type of femtosecond laser offers cutting of materials by ablation without heating the surrounding material. LLNL's Weapons Program presently has a project to study laser cutting techniques. We are applying laser interferometric detection to monitor ultrasonic waves resulting from laser cutting. Information about the ultrasonic wave propagating from the cut can be used as an important diagnostic tool for monitoring cut depth and tracking laser power.

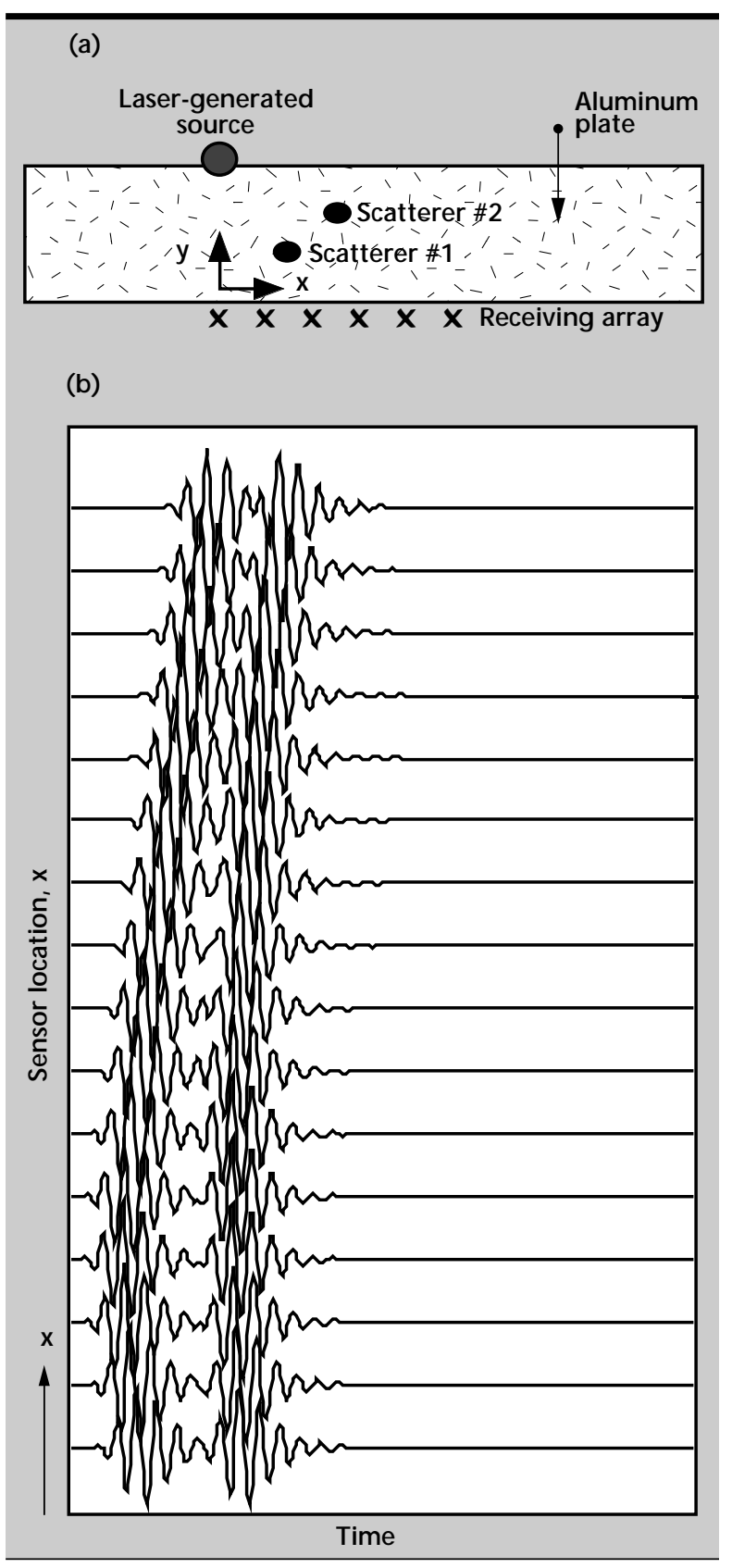

Figure 4. The response due to ideal scatterers in a plate, modeled with the WAVER program. (a) Scatterer \#1 at $(x, y)=(3 \mathrm{~mm}, 2 \mathrm{~mm})$, and scatterer \#2 at $(x, y)=$ (4 mm, $8 \mathrm{~mm}$ ) in an aluminum slab produce an array of sensor responses shown in (b). 
The Michelson interferometer successfully detected ultrasonic waves resulting from the cutting laser. Preliminary laser ultrasonic measurements taken during a cut of stainless steel plates by the femtosecond laser are shown in Fig. 6. Signal (a) is the epicentral response measured on the opposite side of the 8-mm-thick plate from the cutting laser. The compressional wave arrives as expected at $1.2 \mu \mathrm{s}$. Signal (b) is the epicentral response of the 4-mm-thick plate on the opposite side of the plate from the cutting laser.

The response and its multiple reflections from the plate surfaces are measured at $0.5 \mu \mathrm{s}, 1.5 \mu \mathrm{s}$ and $2.5 \mu \mathrm{s}$. Signal (c) is the measured response approximately $5 \mathrm{~mm}$ from the cutting laser on the same side of a 4-mm-thick plate. The low frequency response indicates that a plate wave has been generated by the cutting laser. The cutting laser's short duration pulse and high power generates a very broad band of frequencies with enough energy to excite several wave modes in thin plates. Generation of plate modes is advantageous because these types of waves can travel large distances through a part with minimal attenuation.

By analyzing the arrival time, amplitude and frequency of ultrasonic waves resulting from the cutting laser, cut depth information can be determined. Cut diagnosis can also be derived by the lack of ultrasonic waves propagating in a part, which would indicate cut-through. The amplitude of the ultrasonic signal depends on the amount of material ablated and thus the cutting efficiency. Monitoring the ultrasonic signal during a laser cut is a means of measuring the quality of the cutting laser beam. Both cut-through and laser quality can be used as feedback to the cutting laser. We are presently implementing a prototype system for the laser cutting project.

\section{Summary}

This year we made hardware and software improvements to increase the sensitivity and flexibility of our detection system. Fiber-optic sensing and an improved stabilization circuit increases the adaptability of our interferometer. New data acquisition and scanning capabilities, in addition to implementation of beamforming algorithms, have improved our imaging techniques. Application development for the laser cutting project has helped us become an integral part of LLNL programs.

\section{Future Work}

We will continue to develop laser ultrasonic hardware and software to enhance our imaging capabilities and increase adaptability to LLNL programs. Fiber-optic generation hardware will be designed to combine with our existing fiber-optic detection hardware, thereby completing a fully fiber-based laser ultrasonic system. With a fiber-based system, multiple fibers of different lengths will be used to generate a narrowband signal by sequential pulsing of the fibers. A narrowband signal is detected with better sensitivity than the wideband signals we presently use for generation.

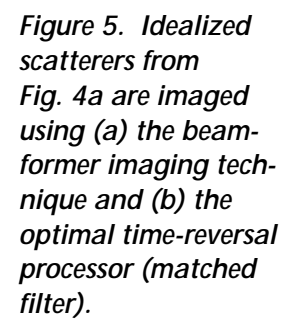

Figure 5. Idealized scatterers from Fig. 4a are imaged using (a) the beamformer imaging technique and (b) the optimal time-reversal processor (matched filter).

(a)

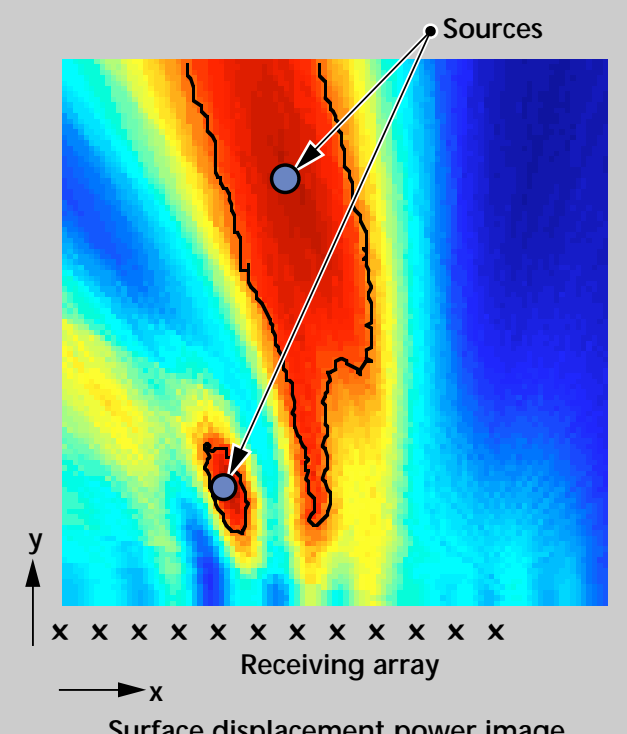

Surface displacement power image (b)

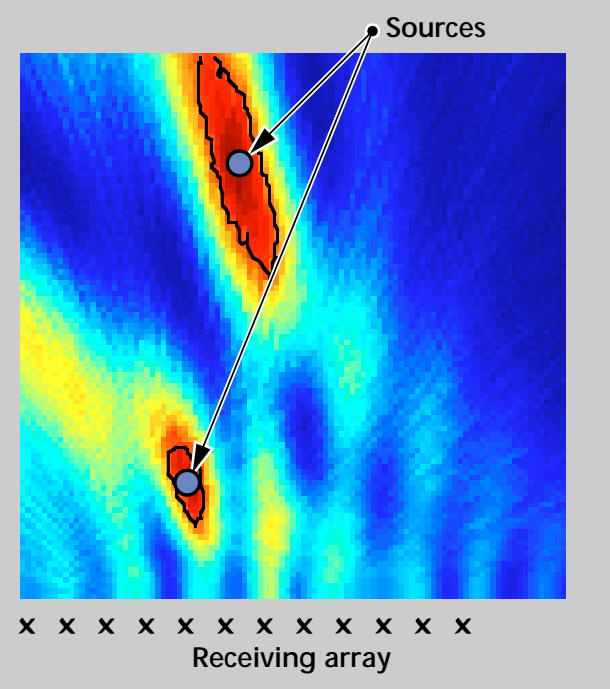

TR processed source localization image 
To develop our hardware capabilities further, we plan to procure a Fabry-Perot interferometer. Fabry-Perot interferometers capture diffuse reflections, yielding better sensitivity in the detection of ultrasonic waves on rough surfaces than the Michelson interferometer.

Software enhancements will expand our processing and imaging capabilities. Addition of a defect scatterer to the WAVER thermoacoustic

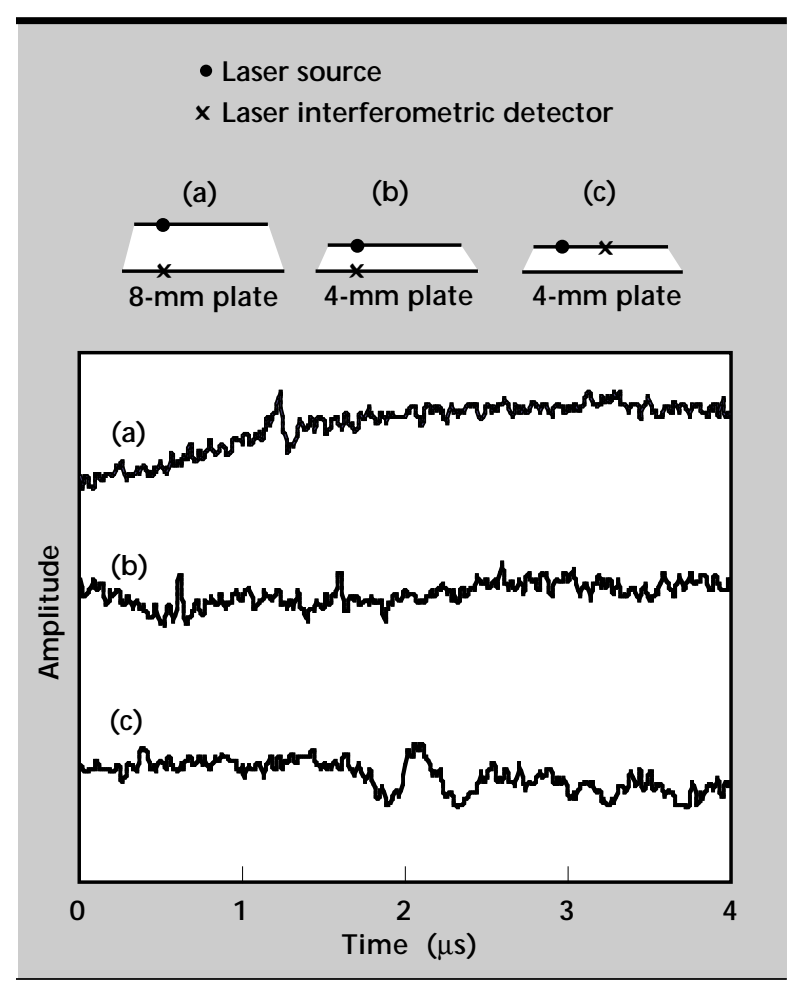

Figure 6. Ultrasonic signals generated by the femtosecond laser cutting a stainless steel plate, detected by the Michelson interferometer on (a) the opposite side of a 8-mm-thick plate; (b) the opposite side of a 4-mm-thick plate; and (c) at a surface distance of $5 \mathrm{~mm}$ from the cutting laser on a 4-mm-thick plate. wave propagation model will allow us to improve the model-based signal processor developed last year.

We will continue work on beamforming algorithms for enhanced imaging. Finally, we will use our expanded thermoacoustic model to determine the optimum collection configuration for sequential fiber-based pulsing.

Next year we will apply laser ultrasonic techniques to determine properties of aging nuclear materials. We will also develop rapid scanning capabilities to monitor tool wear and guide cutting tools in machining parts.

\section{References}

1. Candy, J. V., G. H. Thomas, D. J. Chinn, and J. B. Spicer (1996), "Laser Ultrasonic Signal Processing: A Model-Reference Approach," J. Acoustic. Society Am., 100, (1).

2. Huber, R. D., D. J. Chinn, G. H. Thomas, J. V. Candy, and J. Spicer (1996), "Model-Based Signal Processing for Laser Ultrasonic Signal Enhancement," Review of Progress in Quantitative Non-Destructive Evaluation, Plenum Press, N.Y.

3. Spicer, J. (1991), Laser Ultrasonics in Finite Structures: Comprehensive Modeling with Supporting Experiment, Ph.D. thesis, J ohn Hopkins University, Baltimore, Md.

4. Johnson, D. and D. Dudgeon (1993), Array Signal Processing: Concepts and Techniques, Prentice-Hall, Princeton, New J ersey.

5. Candy, J. V. (1996), Time-Reversal Processing: An Approach to the Scatterer Estimation Problem, Lawrence Livermore National Laboratory, Livermore, Calif., (UCRL-J C-124942). 



\title{
nfrared Computed Tomography for Thermal NDE of Materials, Structures, Sources, and Processes
}

\author{
Nancy K. Del Grande, Philip F. Durbin, and Dwight E. Perkins \\ Manufacturing and Materials Engineering Division \\ Mechanical Engineering
}

\author{
Paul C. Schaich \\ Defense Sciences Engineering Division \\ Electronics Engineering
}

Dennis M. Goodman and David H. Chambers

Lasers Engineering Division

Electronics Engineering

Thomas Milner

Beckman Laser Institute

University of California

Irvine, California

We conducted feasibility demonstrations that established infrared computed tomography (IRCT) as a unique tool for thermal nondestructive evaluation (NDE) of materials, structures, sources, and processes. The IRCT system produced precise, high-definition, time-resolved temperature and thermal inertia maps. These maps depicted thermal-absorption sites in KDP crystals; 3-D images of corrosion damage under aircraft wing fasteners; Zeolite heavy-ion source temperature gradients; U-6Nb temperatures during the forming process; and internal cracks within partially-formed Ti-6Al-4V parts. As a result of leverage from the Lawrence Livermore National Laboratory (LLNL)/Bales small-business CRADA, the IRCT system now provides a high-definition, clutter-free, evaluated, corrosion-defect map in minutes, compared to hours for previous methods. IRCT technology has supported several programs at LLNL and the Idaho National Engineering Laboratory (INEL).

\section{Introduction}

\section{Limitations Of Thermal NDE}

Interpretation of thermal NDE methods is often complicated by clutter, such as unwanted detail. For flash-heated aircraft skins, clutter (for example, from hot spots, sealants, and ripples) is difficult to distinguish from corrosion-thinned aircraft skins. Using a single-band infrared (SBIR) system, we could not distinguish structural defects from environmental clutter such as reflected IR backgrounds from light fixtures, windows, or sky radiation. Using a dual-band infrared (DBIR) system, we removed clutter associated with reflected IR backgrounds to clarify interpretation of structural defects.
Thermal NDE currently specifies the location of structural defects in two spatial dimensions. Threedimensional spatial characterizations of defect types, volumes and depths require a unique approach to the detection, analysis and interpretation of thermal NDE. We call this approach IRCT.

\section{IRCT Concept And Physical Rationale}

IRCT maps precise surface temperatures and bulkmaterial thermal inertias. ${ }^{1-4}$ Surface-temperature maps specify defect size and location. Thermal inertia maps specify defect thickness and depth.

The solution to the heat transfer equation for a thick target with an instantaneous surface heat flux is: 5 


$$
T(x, t)=\frac{q}{\sqrt{4 \pi k \rho c t}} \exp \left(-\frac{x^{2}}{4 \alpha t}\right)
$$

where $T$ is temperature, $x$ is depth beneath the surface, $k$ is thermal conductivity, $\rho$ is density, $c$ is heat capacity, $\alpha$ is thermal diffusivity, $t$ is time and $q$ is surface heat flux. For a semi-infinite solid approximation, the surface temperature is proportional to the inverse square root of time and inversely proportional to the thermal inertia, $\mathrm{P}$, which varies as $(k \rho c)^{1 / 2}$.

\section{The IRCT System}

The IRCT system uses natural (passivelyheated) or pulsed (actively-heated) sources to heat-stimulate structural defects; a dual-band infrared camera to detect them; and rules-based defect-recognition algorithms to evaluate them. Our patented, dual-band IRCT temperaturemapping method, ${ }^{6}$ has ten times better signal-tonoise than conventional thermography, which is a single-band IR temperature-mapping method. We use two IR bands to enhance surface-temperature contrast, and remove spatially-varying, surfaceemissivity noise.

The IRCT system uses VIEW macros on SGI workstations to create masks for automatic defect recognition and clutter rejection. These masks exploit defect depth and volume information specified by time-sequences of thermal inertia maps. Shallow buried objects, defects, and clutter are seen on early-time thermal inertia maps. Deep buried objects, defects and clutter are seen on late-time thermal inertia maps.

\section{Technology Transfer for Aircraft Inspections}

LLNL entered into a Cooperative Research and Development Agreement (CRADA) with Bales Scientific Incorporated (BSI) to commercialize DBIR corrosion-inspection technology. ${ }^{1}$ As part of the CRADA, the LLNL/BSI scientific team produced a single, high-definition, corrosiondefect map for aircraft inspections. The defect map was free of clutter from excess sealants, ripples, and surface features.

The corrosion-defect mapping technique was incorporated into the BSI commercial DBIR scanner, making it easier to access and quantify corrosion damage. Accomplishing the objectives of the CRADA, we upgraded the prototype IRCT system for aircraft and other inspections.

\section{Progress}

\section{Hardware Improvements for Image Quality}

Using a uniform flashlamp source and spectral hood, we improved the IRCT image quality for many of the FY-96 feasibility demonstrations. By removing hot spots equivalent to 1 to $3{ }^{\circ} \mathrm{C}$ temperature differences, we quantified $5 \%$ metal-thickness reductions from corrosion. Because the interior of the spectral hood is highly reflective, more heat reaches the sample from the flashlamps, which improves the signal-to-noise ratio. The hood acts as a blinder for the DBIR camera, by isolating the sample and IR detectors from the environment which surrounded them. This decreases reflected DBIR backgrounds, which reduces the clutter, and clarifies interpretation of the images.

The additional purchase of a $7 X$ close-up lens, with a focal length of $5 \mathrm{~cm}$, will enable us to resolve 25 - to $50-\mu \mathrm{m}$ (1- to 2-mil) defects. This will provide the IRCT system with the potential for high-definition defect maps, derived from thermal and thermal inertia images, which are unsurpassed by any other commercial DBIR system. This has significant implications for characterizing smaller defects in laseroptics components, weapons parts, and canned secondary assemblies. It enhances our capability to support LLNL Programs.

\section{Enhanced Software for Evaluated Defect Maps}

We created an evaluated corrosion-defect map and incorporated the procedure for producing this map into the BSI commercial DBIR system which is used for the IRCT feasibility demonstrations. Also, we developed a unique, rules-based procedure to operate on DBIR temperature, emissivity, and thermal inertia maps. The procedure enables automatic defect recognition and clutter rejection. It tags corrosion defects which affect the structural integrity of the aircraft fuselage. It rejects excess sealants and ripples which have thermal signatures similar to those of corrosion defects.

\section{INVERSE with IR Image Simulator}

We studied a technique to localize absorbers in a medium, using images from an IR camera. This technique ${ }^{7,8}$ has been used successfully to localize capillaries in the skin responsible for port-wine stains. ${ }^{9}$ From a time sequence of IR images, it inverts the heat equation to obtain an estimate of 
the temperature distribution in the material at the time of the first image. This ill-posed problem is solved using a regularization method. 10 The goals of this study were to adapt the INVERSE code for KDP, develop an IR image simulator, and make a preliminary evaluation of its effectiveness using simulated IR images. All of these goals were accomplished.

The IR image simulator was constructed in $M A T L A B$, a matrix algebra and signal processing environment, which calculated the IR irradiance image as a function of time. It was built on the exact expressions developed by Milner, et al. ${ }^{9}$ Simulations of images of point absorbers at various depths and distributions were performed. Typical simulated $3-\mu \mathrm{m}$ IR images, using two point absorbers, separated by $6 \mathrm{~mm}$, at a depth of $3 \mathrm{~mm}$, are shown in Fig. 1. Statistically white noise is added to the images at an rms level of 0.001 of the peak irradiance.

Note the blending of the separate patterns as time progresses. Applying the INVERSE code to the images gives a good estimate of the temperature distribution in the material at the time of the first image $(0.2 \mathrm{~s})$, for depths of $0 \mathrm{~mm}, 1.5 \mathrm{~mm}$, and $3 \mathrm{~mm}$ (Fig. 1b).

\section{Absorption Sites in KDP Crystals}

In Fig. 1, we compare pulsed-laser stimulated (in (c)) and flashlamp-stimulated (in (d)), thermal images of absorption sites in KDP crystals. The laser-stimulated absorption sites were detected in (a)

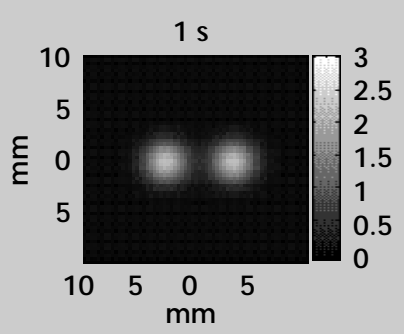

$3 \mathrm{~s}$

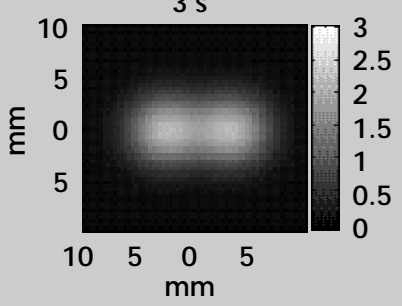

(c)

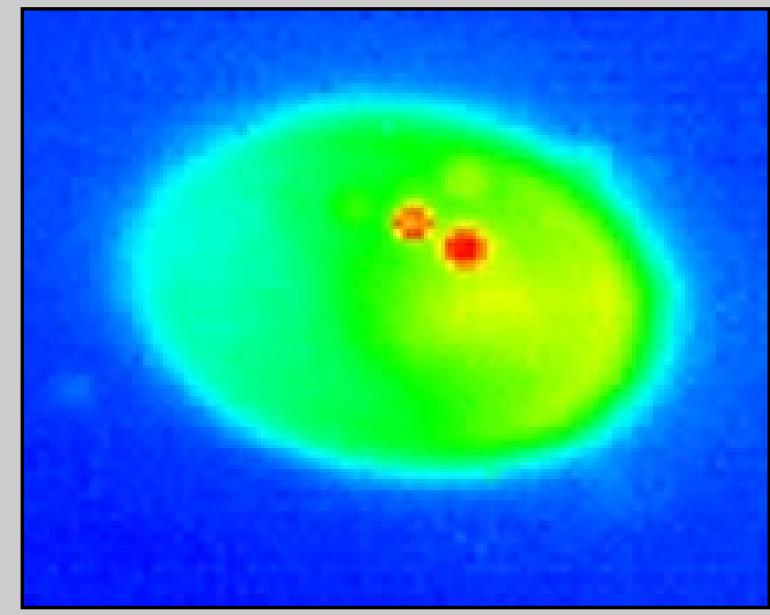

(b)

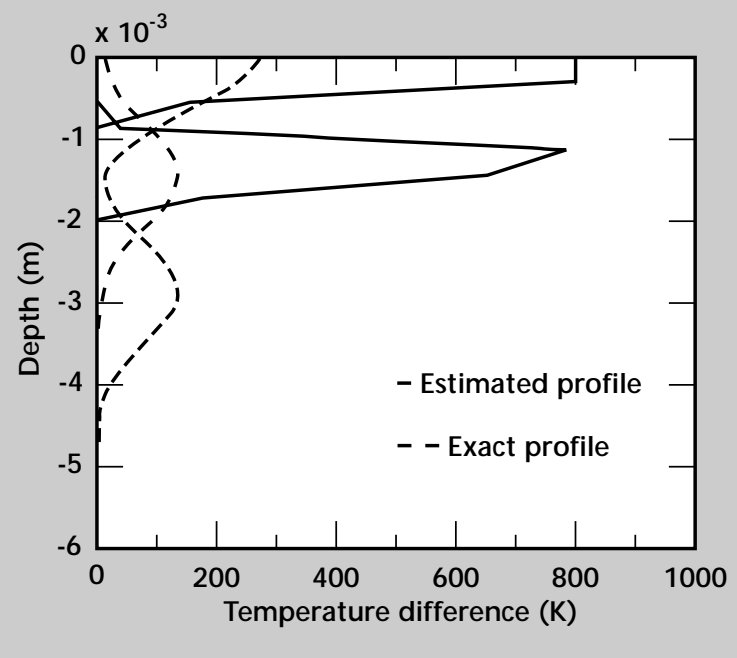

(d)

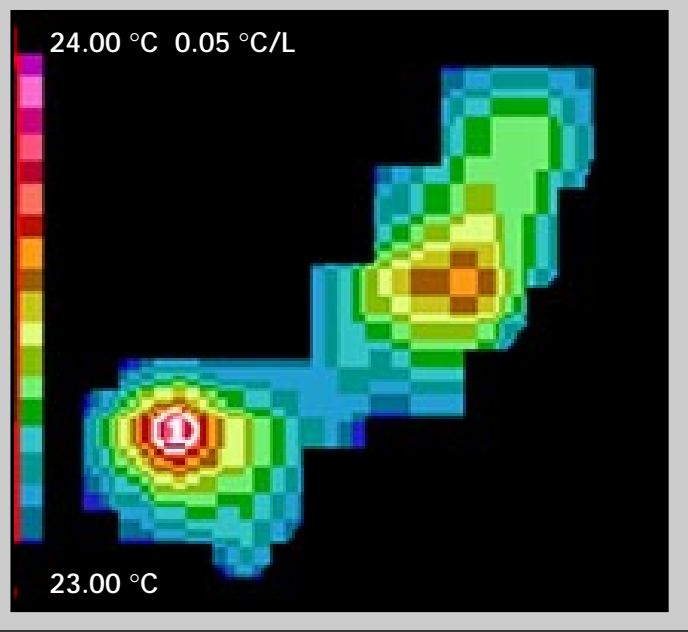

Figure 1. (a) Simulated 3-mm deep point absorbers, separated by $6 \mathrm{~mm}$, after 1, 2, 3, and $5 \mathrm{~s}$; (b) estimated (solid) and exact (dashed) vertical profiles of the temperature distribution at $\mathrm{t}=0.2 \mathrm{~s}$, for initial absorber depths of $0 \mathrm{~mm}, 1.5 \mathrm{~mm}$, and $3 \mathrm{~mm}$, using the INVERSE code; (c) measured thermal images of laser-heated KDP absorption-sites, separated by $0.5 \mathrm{~mm}$, at .002 s after the 150- $\mu$ s heat flash; (d) flash-heated KDP absorption sites, separated by $4 \mathrm{~mm}$, at .040 s after the 4-ms heat flash. 
collaboration with T. Milner at the Beckman Laser Institute, University of California at Irvine. Dr. Milner used an InSb $286 \mathrm{~Hz}$ focal-plane array (FPA) to detect 3- to 5- $\mu \mathrm{m}$ IR absorption-site signals. The thermal image was detected at $2 \mathrm{~ms}$ after the $150-\mu \mathrm{s}, 1.5-\mathrm{mm}$ diameter laser pulse heated the
KDP absorption site differently than the host KDP material. We measured two absorption sites, separated by $0.5 \mathrm{~mm}$, as shown in Fig. $\mathbf{1 c}$.

The IRCT system used a $\mathrm{Hg}$-Cd-Te detector-array to detect the 3- to 5- $\mu \mathrm{m}$ and 8- to $12-\mu \mathrm{m}$ DBIR absorption site signals at $40 \mathrm{~ms}$ after the $4-\mathrm{ms}$,
Figure 2.

(a) Photograph of spin-forming operating system. During the forming operation, U-6Nb part temperature gradients were measured in real time to benchmark code simulations. (b) After the partial-forming operation for Ti-6Al-4V, one flash-heated part had incipient internal cracks, depicted at spots 1,3 , and 4 , leftto-right, within circled areas of the temperature map (c) and relative thermal inertia map (d). (a)

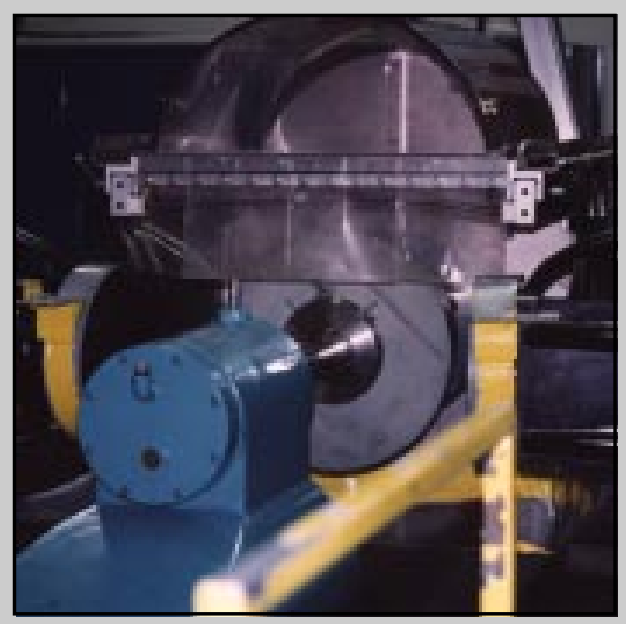

(b)

(c)

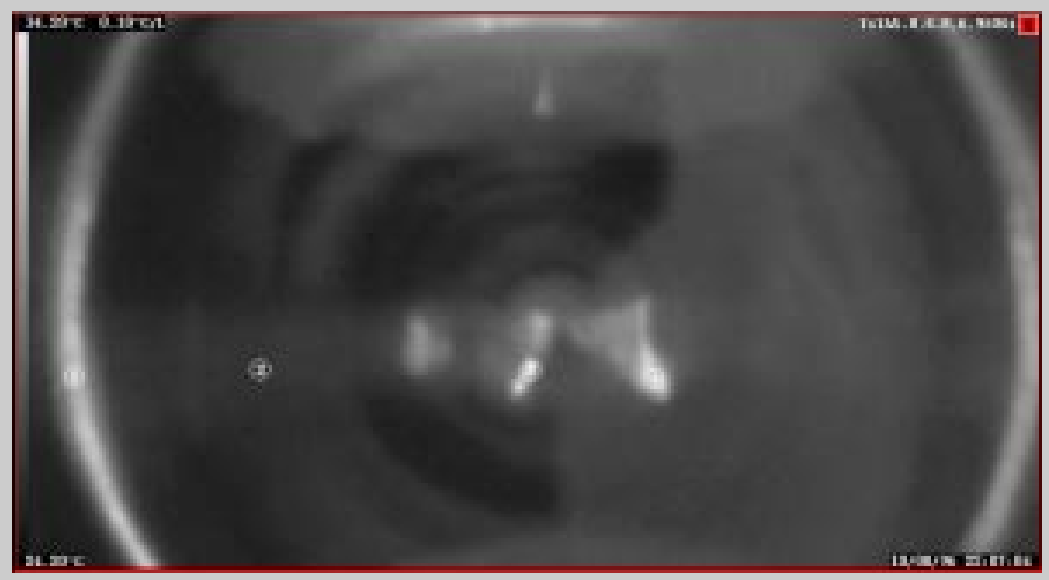

(d)

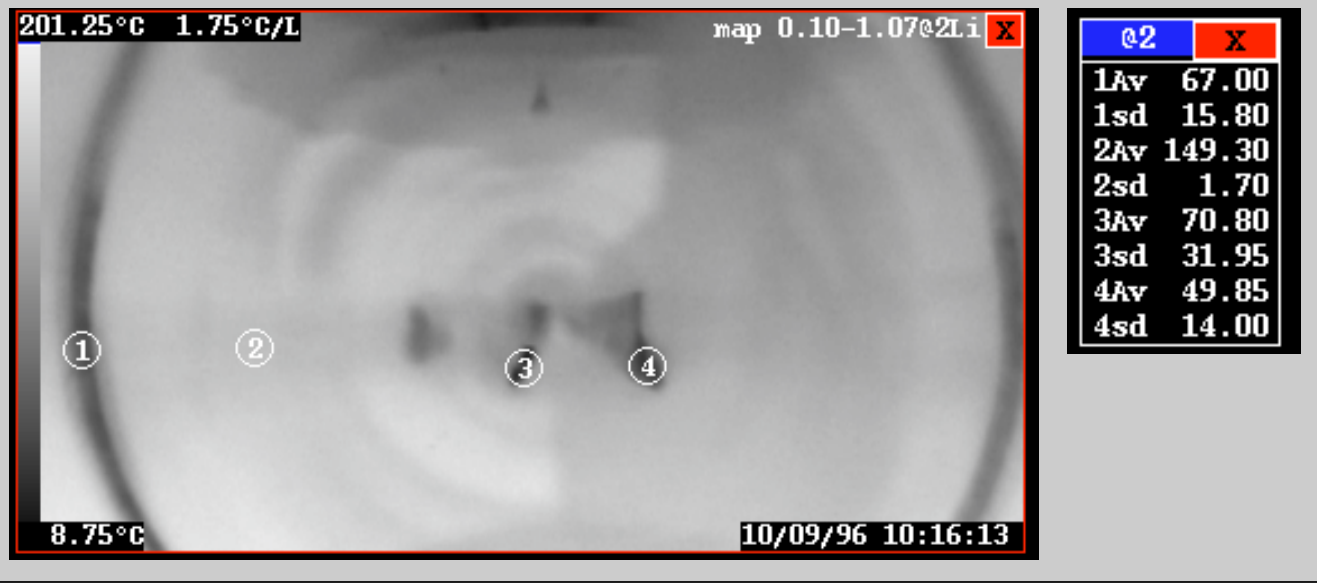

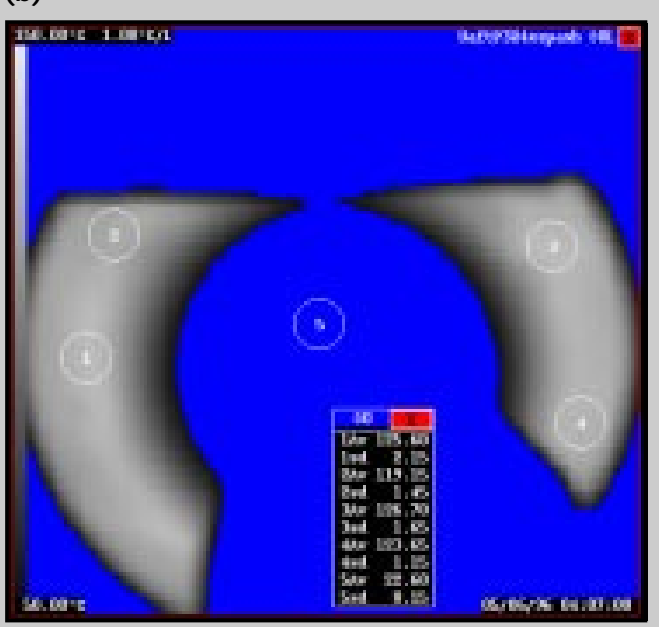

\begin{tabular}{|rr|}
\hline$a 5$ & \multicolumn{1}{|c|}{$\mathrm{X}$} \\
\hline $1 \mathrm{Av}$ & 31.00 \\
$1 \mathrm{sd}$ & 1.10 \\
$2 \mathrm{Av}$ & 25.95 \\
$2 \mathrm{sd}$ & 0.05 \\
$3 \mathrm{Az}$ & 31.05 \\
$3 \mathrm{sd}$ & 2.25 \\
$4 \mathrm{Av}$ & 32.40 \\
$4 \mathrm{sd}$ & 1.05 \\
\hline
\end{tabular}


0.4-m wide flashlamp pulse which heated the KDP crystal absorption site differently than the host KDP material. The DBIR thermal image resolved two absorption sites, separated by $4.0 \mathrm{~mm}$, as shown in Fig. 1d.

Resolution of $0.04-\mathrm{mm}$ absorption sites should be possible using the new $7 X$ close-up lens attachment for the IRCT camera system. We expect to explain vertical depth profiles for absorption-site images in KDP by adapting the INVERSE code for interpreting the experimental data.

\section{Uranium Alloy-Forming Operations Benchmarked}

We successfully monitored a U-6Nb uranium alloy plate while it was being shaped into a device part. Figure 2a shows the spin-forming apparatus. Figure $\mathbf{2} \mathbf{b}$ shows the real-time temperature data which helps scientists better understand changes which occur in the uranium alloy as it undergoes the forming operation. The precise IRCT temperature measurements are used to benchmark numerical simulations.

\section{Internal Cracks Detected in Partially-Formed Titanium Alloy}

We flash-heated a partially-formed Ti-6Al-4V part which had a smooth surface with no visible cracks. The thermal map in Fig. 2c, and thermal inertia map in Fig. 2d, show incipient internal cracks at the 14-in. diameter location where large visible cracks occurred in a similar, Ti-6Al-4V alloy sample. The thermal map depicted anomalous cooling rates at sites where the thermal inertia map had very little resistance to temperature changes. Incipient internal cracks may have developed during the forming operation that was conducted with the equipment shown in Fig. 2a. We also detected unexplained cooling-rate anomalies near the center of the uncracked sample.

\section{Corrosion Damage Found under Aircraft Wing Fasteners}

We measured corrosion damage under fasteners in a KC-135 aircraft wing panel from Tinker Air Force Base. Early-time $(0.5 \pm 0.4 \mathrm{~s})$ minus latetime $(1.7 \pm 0.6 \mathrm{~s})$ temperature-difference maps quantify the relative volume of corrosion damage under two aircraft wing fasteners, shown in Fig. 3a, and 13 aircraft wing fasteners, shown in Fig. $3 \mathbf{b}$. Sites which have the most white areas have the highest peaks and the largest volumes of metalthickness reduction from the corrosion damage.

\section{Zeolite Source Temperature Gradients Measured}

We measured the temperature of the recirculator heavy ion source at 500 to $1200 \mathrm{~K}\left(230\right.$ to $\left.930{ }^{\circ} \mathrm{C}\right)$. The Zeolite target is the source of potassium ions produced in the small LLNL recirculator. The recirculator is a prototype recirculating induction accelerator under development by LLNL's Heavy Ion Fusion (HIF) Group. By imaging the source operating temperatures, and temperature gradients, information may be gleaned about the quality of the heavy ion beam. Our temperature measurements provided assurance that the recirculator heavy ion source was performing as expected.

We used a Ag mirror, and a ZnSe (vacuum barrier) window, which had a combined reflectance and transmittance of $75 \%$ of the signals in the shortwave (SW, $5 \pm 0.5 \mu \mathrm{m}$ ), and longwave (LW, $10 \pm 1.5 \mu \mathrm{m})$ IR bands. The emitting surface behaved like a selective radiator. It had emissivity differences in the LW and SW bands which may be related to silicate build-up from oxidation. Silicates have a broad molecular resonance at

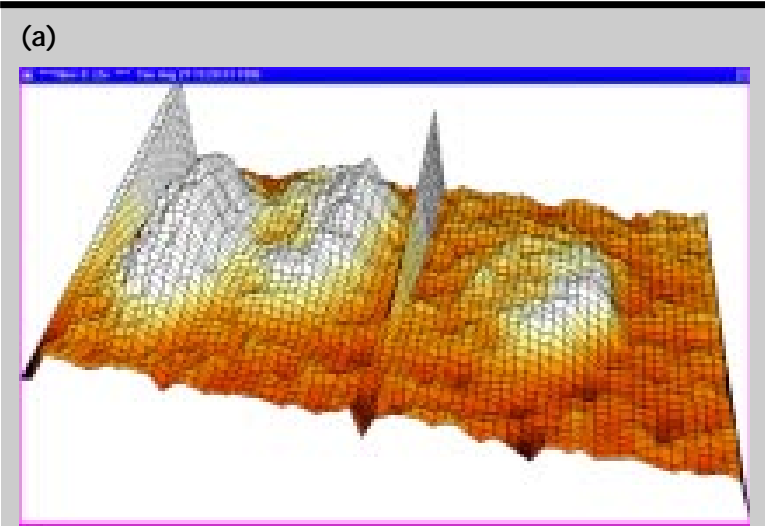

(b)

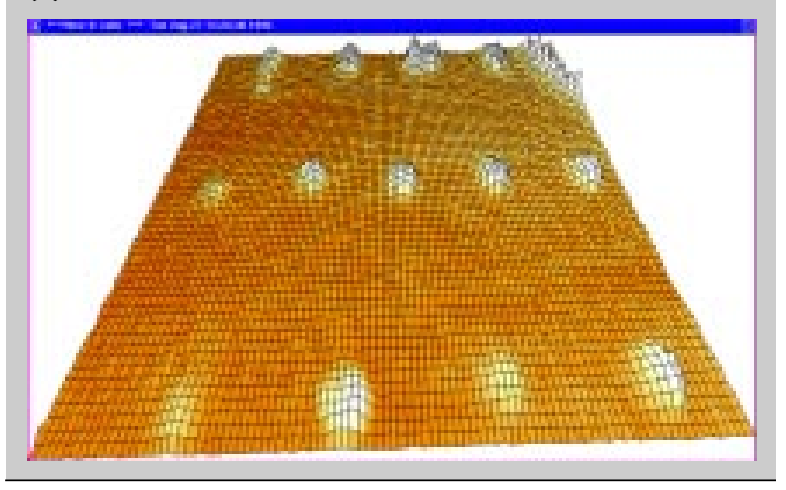

Figure 3. Three-dimensional displays of early-time minus latetime temperature-difference maps, which quantify the metalloss volume from intergranular corrosion, where the damage is (a) $4 \times$ larger under the left, compared to the right aircraft wing fastener, and (b) varies under thirteen wing panel fasteners. 
$10 \mu \mathrm{m}$, which does not affect the SWIR temperature measurement. The LWIR temperature nonuniformities for the Zeolite emitting source are shown in Fig. 4.

\section{Summary}

IRCT applications conducted during FY-96 generated significant interest in the potential of IRCT to address key issues for major LLNL Programs.

The National Ignition Facility (NIF) Program is interested in the potential of high-resolution IRCT measurements, in conjunction with INVERSE simulations, to correlate KDP thermal absorption-site locations, sizes, and depths of origin, with known
KDP defects, using the NIF laser-diagnostics table and damage testing system.

The Advanced Development and Production Technologies (ADaPT) Program is interested in IRCT technology to benchmark the forming operations for weapons parts. The initial tests succeeded in monitoring the spin-forming process temperatures in real time for U-6Nb parts.

The Enhanced Surveillance Program (ESP) is interested in the capability of thermal and thermal inertia maps to determine the type, location, size, and depth of dimensional changes in plutonium weapons components, and in corrosionrelated dislocations, or migrations, in canned secondary assemblies.
Figure 4. Thermal images of the 1-in. diameter Zeolite heavy ion source SWIR images at 4.5 to $5.5 \mu \mathrm{m}$ (left column), and LWIR images at 8.5 to $11.5 \mu \mathrm{m}$ (right column). The measured average temperatures assume an ideal black body source with emissivity $\varepsilon=1.00$ in (a), (b), (c), and (d); a window transmission $\tau=1.00$ in (a) and (b); $\tau=0.75$ in (c), (d), (e), and (f); a selective-radiator source with a SWIR emissivity, $\varepsilon=0.99$ in (e); and LWIR emissivity $\varepsilon=0.89$ in (f). The measured average temperature values and standard deviations apply to source sites depicted by the line profiles.

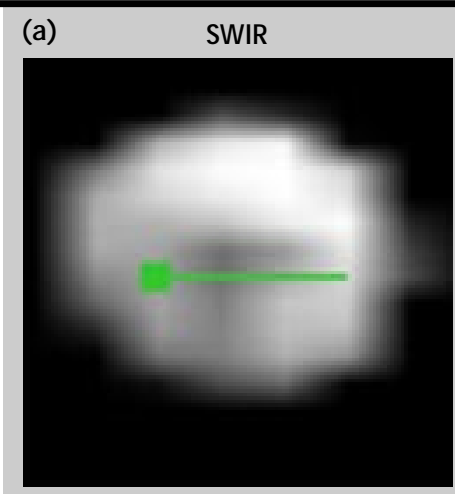

Average $\mathrm{T}=799 \pm 2^{\circ} \mathrm{C}$

(c)

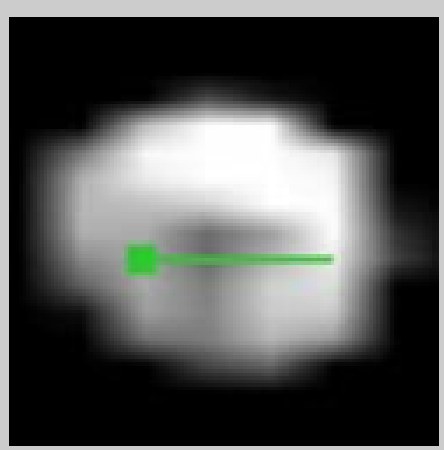

Average $\mathrm{T}=911 \pm 2^{\circ} \mathrm{C}$

(e)

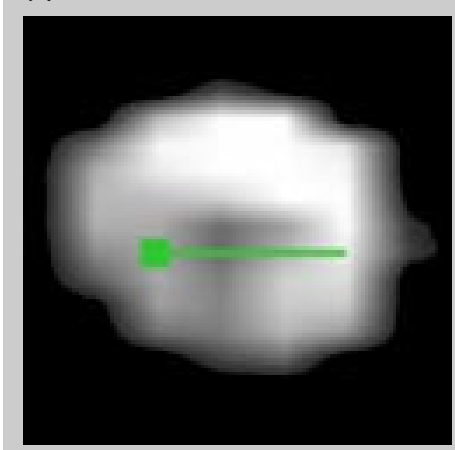

Average $\mathrm{T}=915 \pm 2{ }^{\circ} \mathrm{C}$

\section{$802{ }^{\circ} \mathrm{C}$}

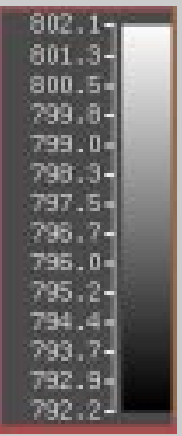

$792{ }^{\circ} \mathrm{C}$

$914{ }^{\circ} \mathrm{C}$

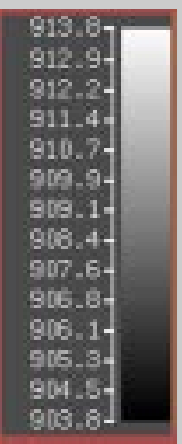

$904{ }^{\circ} \mathrm{C}$

$918{ }^{\circ} \mathrm{C}$

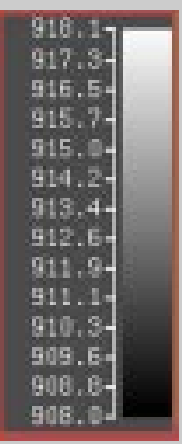

$908{ }^{\circ} \mathrm{C}$

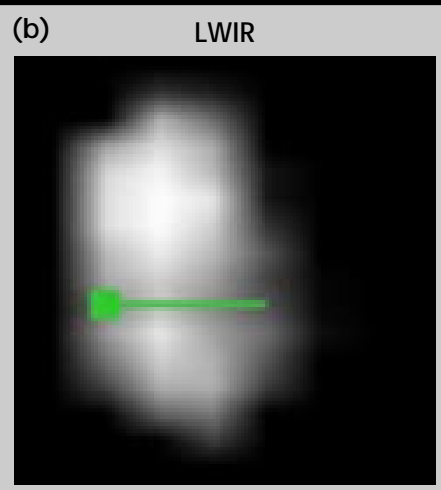

Average $\mathrm{T}=648 \pm 2{ }^{\circ} \mathrm{C}$

(d)

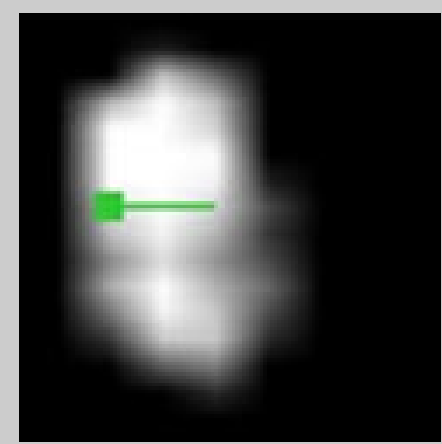

Average $\mathrm{T}=822 \pm 2^{\circ} \mathrm{C}$

(f)

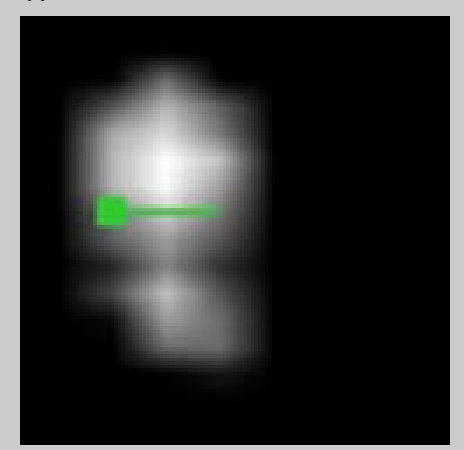

Average $\mathrm{T}=911 \pm 2{ }^{\circ} \mathrm{C}$ $652^{\circ} \mathrm{C}$

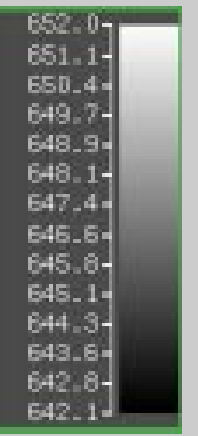

$642{ }^{\circ} \mathrm{C}$

$826^{\circ} \mathrm{C}$

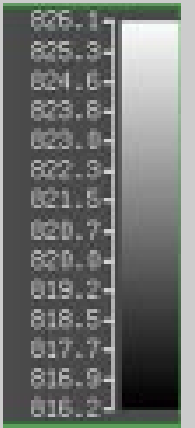

$816^{\circ} \mathrm{C}$

$918{ }^{\circ} \mathrm{C}$

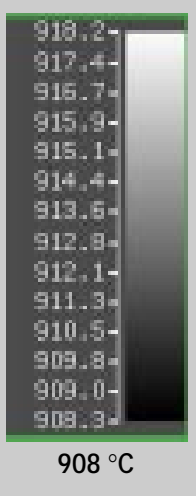


LLNL's and LBL's HIF teams need precise, hightemperature and temperature-gradient measurements to characterize the Zeolite heavy ion source. By monitoring aging and build-up of source oxides, the source can be replaced, as needed, to provide assurance of heavy-ion beam quality.

INEL is currently sponsoring a demonstration of the IRCT technology to measure wall-thickness reductions for corroded waste containers. Successful results would produce spin-off applications for a variety of environmental and hazardouswaste projects inside and outside INEL.

\section{Future Work}

We are planning additional proof-of-principle experiments to test the potential of new IRCT applications in support of LLNL programs. Our goals to address key program issues are discussed below.

For the NIF Program. We would test the capability of the high-resolution IRCT system to image flash-heated KDP absorption sites as small as $40 \mu \mathrm{m}$; adapt the IRCT system for imaging laserheated KDP absorption sites in NIF environments; apply the INVERSE code to interpret their origin, develop a state-of-the art thermal diagnostic for the NIF damage-testing system.

For the ADaPT Program. We would test the predictability of design calculations for weaponscomponent forming operations; monitor forming operations in real time; develop image-processing procedures to improve the image display; and quantify the results.

For the ESP Program. We would make the existing IRCT system more rugged for operating in weapons system environments; test the capability of IRCT thermal and thermal inertia images to characterize internal gaps in canned secondary assemblies; and investigate dimensional changes in mock and actual plutonium weapons parts.

For the HIF Program. We would adapt the highresolution IRCT system to measure precise heavy ion source temperatures and temperature gradients at 200 to $1000{ }^{\circ} \mathrm{C}$; and provide co-registered, DBIR digital data for image analysis, display, and processing on suitable work stations.

\section{Acknowledgments}

We acknowledge the forming-operation measurements and Ti-alloy samples provided by $\mathrm{S}$. Torres of Chemistry and Materials Sciences, and the recirculator heavy ion source measurements by $M$. Nelson of the HIF Group. We are grateful for the KDP samples provided by M. Yan and D. Aikens of Lasers Division. We thank G. Gallegos for valuable technical discussions, and $\mathrm{H}$. Martz for his technical support.

\section{References}

1. Del Grande, N. (1996), "Dual-Band Infrared Computed Tomography: Searching for Hidden Defects," Science and Technology Review, University of California, Lawrence Livermore National Laboratory, Livermore, Calif., (UCRL-52000-96-5), p. 23.

2. Del Grande, N. K., P. F. Durbin, and D. E. Perkins (1996), "Infrared Computed Tomography For Characterizing Structural Defects," Nondestructive Evaluation Thrust Area Report, Engineering Research, Development and Technology FY95, H. E. Martz, Ed., Lawrence Livermore National Laboratory, Livermore, Calif., (UCRL 53868-95), February.

3. Del Grande, N. K., and P. F. Durbin (1995), “Mapping hidden aircraft defects with dual-band infrared computed tomography," Tobey Cordell, Ed., Proceedings of SPIE Conference 2455: Nondestructive Evaluation of Aging Aircraft, Airports, Aerospace Hardware, and Materials, Oakland, Calif., J une, pp. 82-93.

4. Del Grande, N. K., and P. F. Durbin(1995), "Stimulated dual-band infrared computed tomography: a tool to inspect the aging infrastructure," B. F. Andreson and M. S. Scholl, Eds. Proceedings of SPIE Conference 2552: Infrared Technology XXI, San Diego, Calif., J uly, pp. 292-301.

5. Carslaw, H. S., and J. C. J aeger (1980), Conduction Of Heat In Solids, Second Edition, Oxford Univ. Pr., London, England, pp. 101, 112, and 259.

6. Del Grande, N. K., P. F. Durbin, K. W. Dolan, and D. E. Perkins (1995), Emissivity Corrected Infrared Method For Imaging Anomalous Structural Heat Flows, (Patent No. 5,444,241). 
7. Milner, T. E., D. M. Goodman, B. S. Tannenbaum, B. Anvari, L. O. Svaasand, and J. S. Nelson (1996), "Imaging Laser-Heated Subsurface Chromophores in Biological Material: Determination of Lateral Physical Dimensions," Phys. Med. Biol., 41, pp. 31-44.

8. Milner, T. E., D. M. Goodman, B. S. Tannenbaum, and J. S. Nelson (1995), "Depth Profiling of Laser-Heated Chromophores in Biological Tissues by Pulsed Photothermal Radiometry," J. Opt. Soc. Am. A, 12 (7), pp. 1479-1488.
9. Milner, T. E., D. J . Smithies, D. M. Goodman, A. Lau, and J. S. Nelson (1996), "Depth Determination of Chromophores in Human Skin by Pulsed Photothermal Radiometry," Appl. Opt., 35, (19), pp. 3379-3385.

10. Goodman, D. M., E. M. Johansson, and T. W. Lawrence (1993), "On Applying the ConjugateGradient Algorithm to Image Processing Problems," Multivariate Analysis: Future Directions, C. R. Rao, Ed., pp. 209-232. 


\title{
utomated Defect Detection for Large Laser Optics
}

\author{
Laura N. Mascio \\ Engineering Research Division \\ Electronics Engineering \\ Clint M. Logan \\ Defense Technologies Engineering Division \\ Mechanical Engineering \\ Harry E. Martz \\ Manufacturing and Materials Engineering Division \\ Mechanical Engineering
}

We have developed and implemented automated defect detection software for round large laser optics imaged by the Beamlet Lens Inspection System. In pursuit of this goal, we encountered several extensions to the original problem. As a result, progress has been made towards an automated inspection of more complicated square lens images on Beamlet, and towards estimating the image processing needs for the Lasers Program and the National Ignition Facility project at Lawrence Livermore National Laboratory.

\section{Introduction}

The National Ignition Facility (NIF) at Lawrence Livermore National Laboratory (LLNL) faces enormous challenges in the inspection and maintenance of thousands of large laser optics. Optics inspection must occur at several stages: 1) off-line contamination detection to meet cleanliness standards before assembly; 2) on-line (in situ) damage detection, especially at vacuum barriers, to ensure safety during operation; and 3) surface quality inspection and defect characterization for quality control.

This FY-96 project was originally proposed to address a more modest and specific problem of automating the tedious manual inspection process used to document the laser damage and other defects on a round Beamlet lens between operations (off-line). That goal was quickly addressed by developing and implementing automated inspection software to analyze video camera images of the lens. Because a digital acquisition system had been installed on-line, these images could be acquired and analyzed without removing the lens from the beamline. The defect detection software is currently operational and can be executed from the Beamlet control room.
During development of that software, many extensions of the original problem became apparent, and this project began to address them. The first extension involved analyzing newly available on-line image data acquired from a square lens on Beamlet. These images are more complicated in that reflections and peripheral hardware are visible within the area of the lens. Algorithms for background suppression and defect enhancement have been developed to assist in analyzing these images.

Further extensions of the original goal included investigating various future requirements of LLNL's Lasers program and the NIF project in the area of optics inspection. This investigation was done in conjunction with personnel who are designing the contamination control, optics damage inspection and quality control in support of NIF.

\section{Progress}

\section{Automating the Manual Inspection Process}

The original goal of the project was to automate the tedious manual inspection of large laser optics, 
which was done by a technician in a dark room using a flashlight and clipboard. Defects were laboriously measured and recorded as shown in Fig. 1.

When this project got under way, an on-line image acquisition system (called the "Beamlet Lens Inspection System") had already been installed such that a round (58 cm diameter) sol-gel AR coated fused silica lens in the $L 3$ position on Beamlet could be imaged in place between laser shots. A video CCD camera $(640 \times 480$ pixels with 8 bits of grayscale per pixel) was fixed in place below the optic and out of the beam path (Fig. 2). Between 3 and 8 quarter-inch fiber optic bundles were used to inject light into the edge of the optic. Images of this optic benefit from the dedicated acquisition conditions and from minimal clutter in the field of view.

\section{Algorithm for Detecting the Optic}

Any defects in the optic manifest as irregularities in the acquired image, due to the light scattered at the defect site. Figure $\mathbf{3}$ shows defects that have been computationally detected by the method to be described. The first step towards defect detection was to write algorithms to isolate the optic from the rest of the image. In the case of the round lens, the image shows an elongated lens because the camera position is below the optic and the line of view is not at normal incidence to the optic. The Circle Hough Transform (CHT) ${ }^{1}$ was applied because it is robust in the face of noisy, partially obscured or partially missing data, and is significantly faster than the equivalent transform for ellipses.

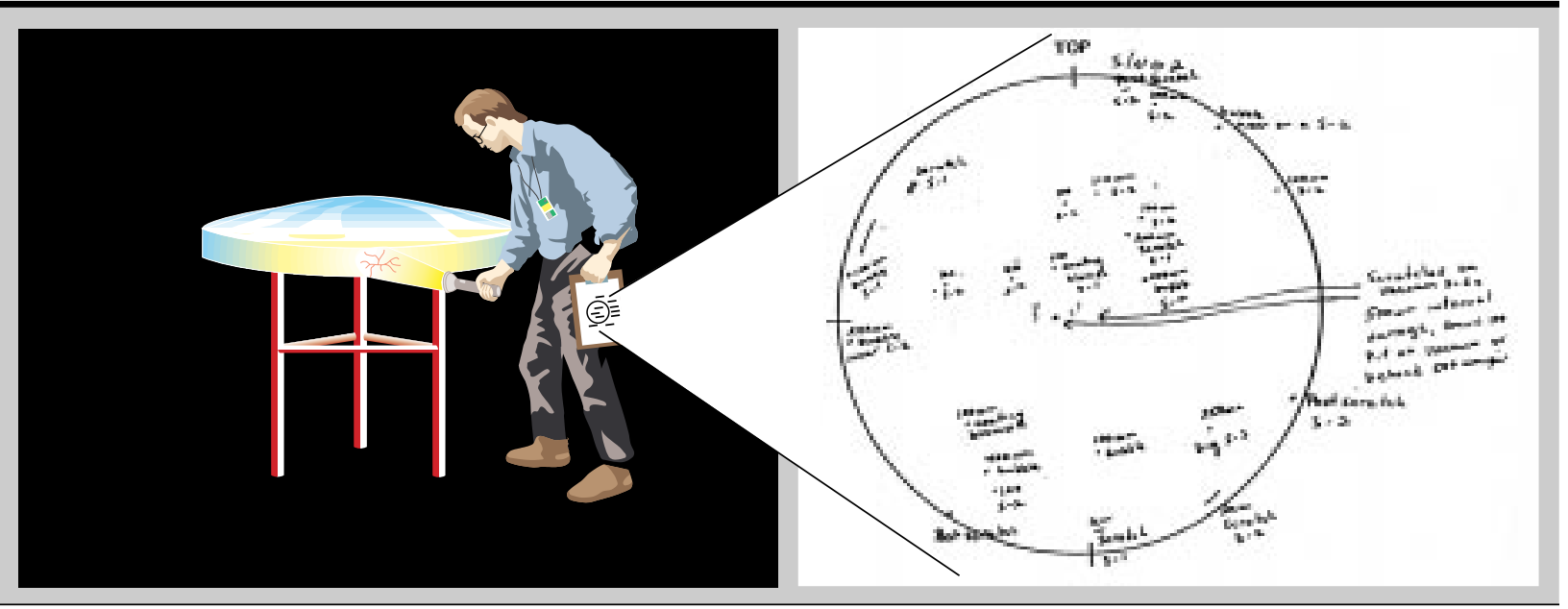

Figure 1. Illustration of the tedious manual inspection process, where defects were laboriously measured and recorded over time.

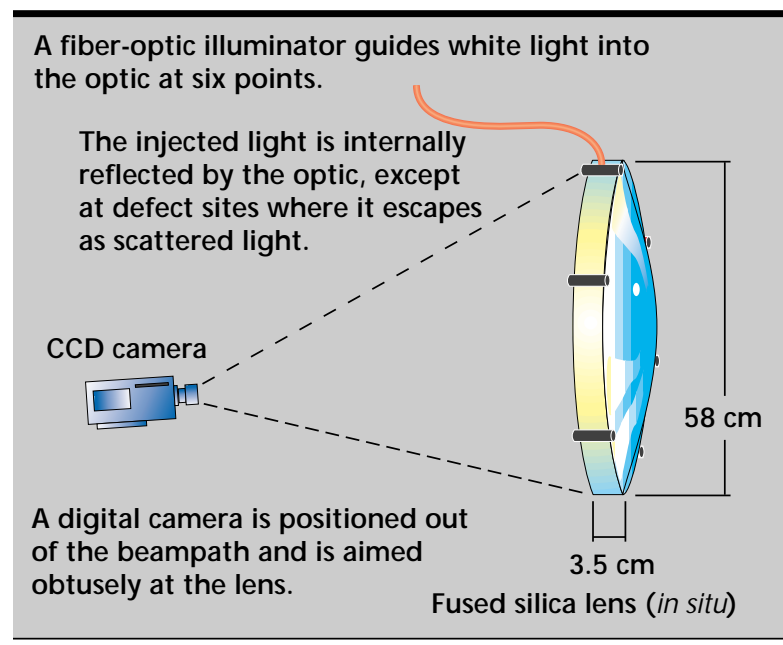

Figure 2. Schematic of CCD video camera providing dedicated acquisition of the entire $L 3$ round lens on Beamlet. The camera is positioned below the lens and at an angle. Fiber optic guides feed white light into the optic for illumination.

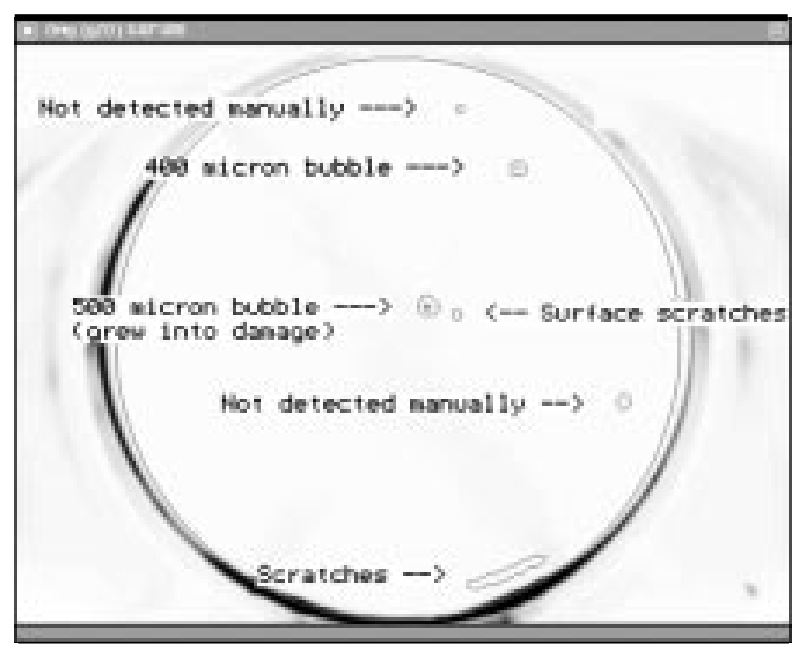

Figure 3. Automatic detection of the boundary of the optic (large circle) and defects within the optic (small, irregular contours). Manual inspection indicated the size and type of each detected defect as labelled. 
The basic premise of the $\mathrm{CHT}$ is that each point (from an edge magnitude image) along a circle can be transformed into a line in Hough parameter space (3-D: $x$ coordinate, y coordinate and radius) such that each point of the line "votes" for the location (center $x$ and $y$ coordinates and the radius) of the circle. When the transformation is complete, the maximum number of votes in Hough space reveals the center and radius of the circle. The $\mathrm{CHT}$ is summarized in Fig. 4.

The CHT consistently and reliably found the best circle that fit the elongated lens data, and effectively isolated the area of the optic from the surrounding image data for all 83 available test images, from December, 1995 to September, 1996. An example of the $\mathrm{CHT}$ result is illustrated as the large complete circle in Fig. 3.

\section{Algorithms for Detecting Defects}

Once the region of the lens was isolated, algorithms were applied within that region for defect detection. The developed method is based on edge magnitude, since gradients are detectable independent of the choice of illumination (dark field or bright field). First, a Sobel differential filter is applied to the image by computing the square root of the sum of the squares of the gradient in the horizontal and vertical directions. Then, a histogram of the Sobel result is computed, which charts the function of intensities (edge magnitude) versus the rate

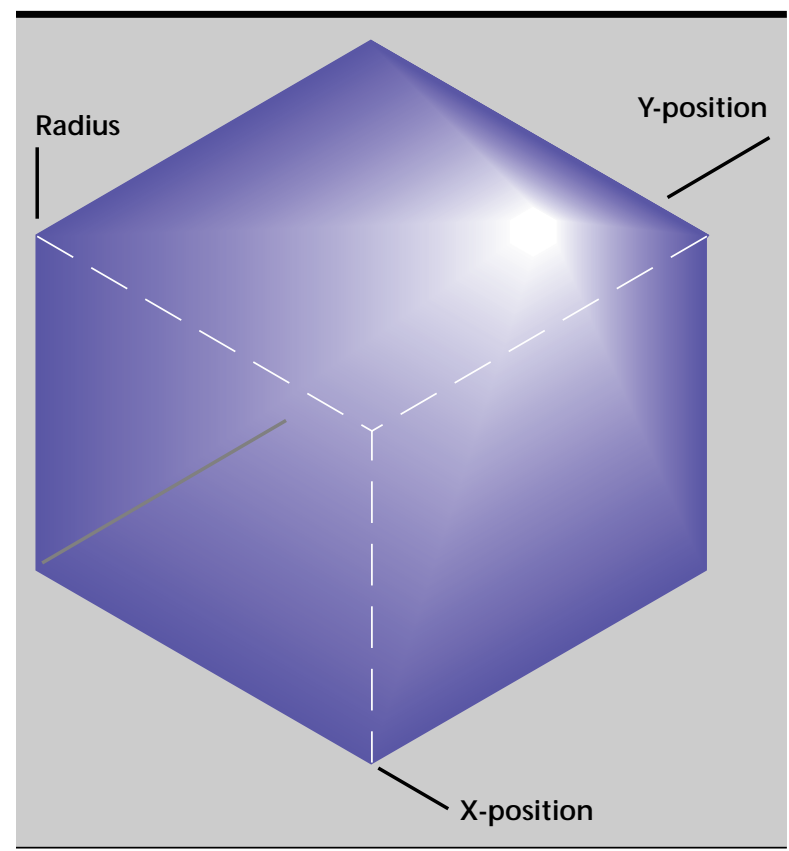

Figure 4. Summary of the Circle Hough Transform. of occurrence for each intensity in the image. This histogram is computationally analyzed using a triangular construction method to determine the threshold level, a single intensity value below which there is no useful defect information. The triangular construction algorithm computes the clipping level to be a constant offset below the knee of the "triangular" peak formed by the background at the low intensity end of the histogram.

Applying the computed threshold level to the image yielded a binary mask that has "on" pixels where the defects lie and "off" pixels elsewhere. This binary image is then manipulated using binary morphology functions to fill in any holes within the area of defects. These areas can be measured for various features, which will be addressed later. Finally, the defect areas are dilated and contoured so the contour can be placed over the original image for displaying the results that were shown in Fig. 3.

\section{Algorithms for Visual Enhancement of the Area}

Figure 5 shows the same optic using a severe local contrast enhancement. This is useful in that it is otherwise extremely difficult to discern defects visually in the original image. It provides a visual aid for the operator, and acts as a quality check when the optic cannot be inspected manually. The defects that were computationally detected and marked in Fig. $\mathbf{3}$ can be visualized as abnormalities

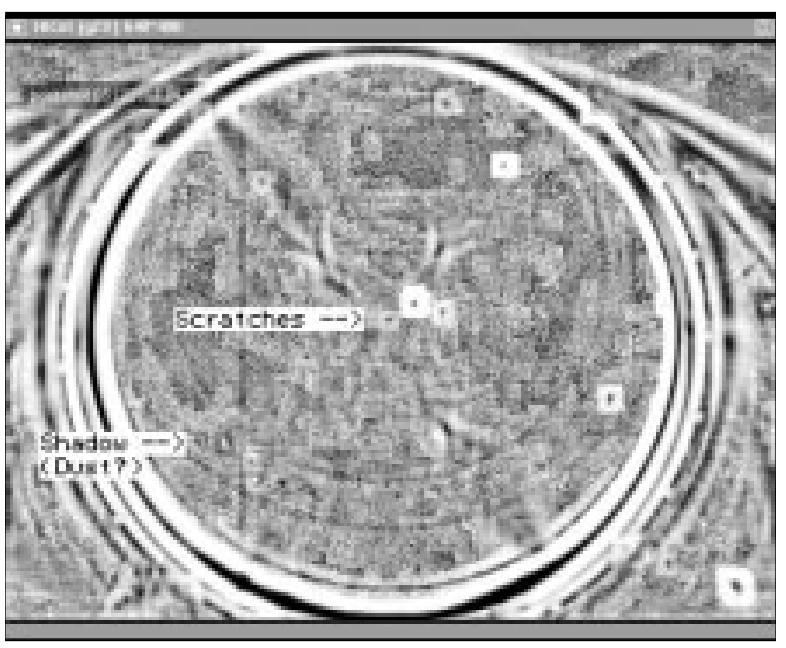

Figure 5. Visual quality check for fine detail that is not visible in the original image. The two subtle abnormalities indicated are visible here but were not detected by the automated detection scheme demonstrated in Fig. 3. 
here, where they were not necessarily visible in Fig. 3. The additional defects visible in Fig. 5 (a shadow and some scratches) are small subtleties which had been detected by manual inspection but were not considered alarming. Other smaller blemishes that had been detected by manual inspection were not resolvable with any of the developed enhancement of detection schemes.

The method developed to create this high contrast image consists of a $13 \times 13$-pixel moving kernel that travels across the image in a raster fashion. At each placement of the window, the 169 corresponding intensities in the image are ranked in order from darkest to brightest. These are then mapped so that the darkest corresponds to 0 , the brightest corresponds to 255 (to cover the 8 bit dynamic range of the display) and the rest are linearly mapped in between. The result from each position is that the intensity of (only) the center pixel in the result image is reassigned to its corresponding "stretched" value from the linearly mapped values. The other pixel values are unaltered until they are at the center of the moving kernel.

\section{Defect Detection Results}

For these images, manual inspection results were available to compare with the computer detection results. The manual inspection results recorded all minute blemishes on the lens and revealed that the defects on this lens ranged in size up to $500 \mu \mathrm{m}$.

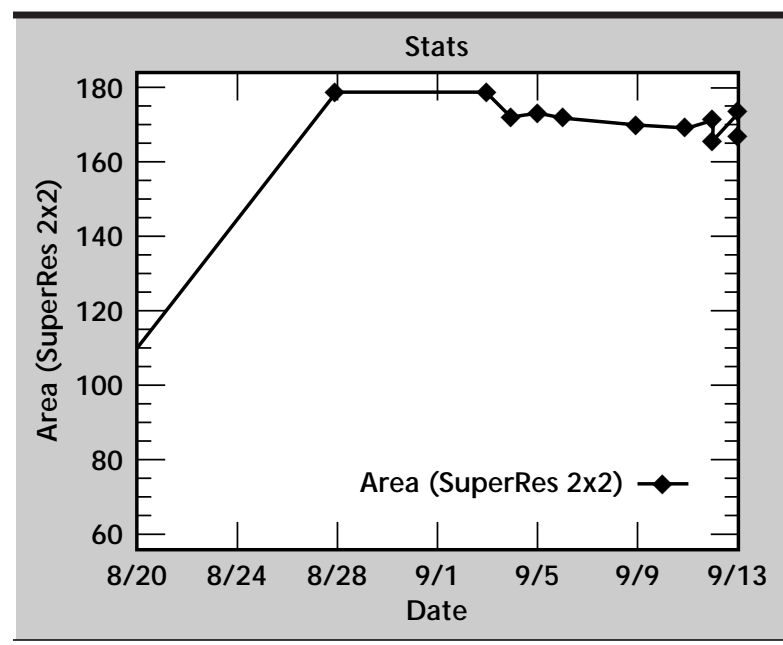

Figure 6. Plot of the area of the detected signal for the largest defect on the round lens vs time. The initial jump in the signal area was due to a scheduled camera focus adjustment. The relatively stable area measurement on subsequent days indicates little change in area of the physical defect and concurs with control room records that acquisition conditions were kept stable.
The required critical detection size for Beamlet is on the order of 1 to $2 \mathrm{~mm}$. The critical flaw size for NIF will likely be even larger.

The computer detection scheme consistently detected the larger of these objects, but inconsistently detected the smaller of them. Some of the smaller defects would be detected, then "disappear" in a subsequent image and then later "reappear". The reason for this is that the quality of the images varied due to different acquisition parameters, especially camera focus, and so their amenability to analysis varied as well. The fine detail information content among images was inconsistent. Since this project only worked retrospectively on archived images, it was not possible to control the image acquisition parameters. Nonetheless, the developed algorithms were able to consistently detect the 400 to $500 \mu \mathrm{m}$ bubbles and some scratches in all available test images.

It should be noted that computer detection of defects is dedicated to locating defects. It does not necessarily preserve their shape or size. To measure the defects, they must be analyzed quantitatively after detection, as described below.

\section{Defect Measurements}

The camera resolution for these images is about $1.3 \mathrm{~mm}$ per pixel, varying across the image due to various optical distortions. But due to defocus and saturation during acquisition, and due to diffraction at the defect site, the visible signal (spot) on the image can be much larger than the physical defect, which itself may not be resolvable. For example, a defect on the order of $500 \mu \mathrm{m}$ in diameter scattered light to form a spot that was 7 pixels (approximately $9 \mathrm{~mm}$ ) in diameter in the image. Because of this effect, measurements made on the resulting spot in the image only serve to characterize the distorted/diffracted light signal and not necessarily the absolute physical properties of the defect. The relationship between the scattered light signal and the absolute physical properties of the defect were not studied here. Relative measurements, however, on the defect signal over time reflected relative physical changes in the environment.

Figure 6 shows how a change in camera focus drastically altered the area of the defect signal for the $500 \mu \mathrm{m}$ bubble near the center of the round lens around August 23. It also shows how the visible signal did not change size over the next subsequent shots, assuming the acquisition conditions stayed constant over that time, which is in concurrence with acquisition records from the Beamlet control room. 
The plotted measurement values were attained by first "reconstructing" the original analog signal by multiplying each pixel of the sampled data by a sinc function. ${ }^{2}$ This increases the resolution of the data so that each pixel becomes $2 \times 2$ pixels and the number of pixels across the diameter of the object is approximately doubled. For small defects, this has the effect of decreasing the error of the area measurement from $10 \%$ to $1 \%{ }^{2}$ The next step is to use grayscale morphology to subtract the background from the image so variations in illumination across the image will be leveled. Then, a triangular threshold algorithm, described above in the section on defect detection, was applied to determined the cutoff intensity that separates the defect from its surroundings. The area is then determined by counting the number of pixels resulting from the threshold. This area can be converted to physical units if the resolution at the site of the defect is known.

\section{Extension to a Final Focus Square Lens}

Later in the year, images from a square $(40 \mathrm{~cm} \times 40 \mathrm{~cm})$ sol-gel AR coated wedged fused silica lens became available from the final focus lens of the Beamlet Lens Inspection System. These images also benefited from having dedicated illumination and camera, but the image is acquired through a mirror which is moved in and out of position between shots, which may cause sub-pixel registration offsets between images. More light is pumped into this square lens, and thus more light spills out of it, illuminating the surroundings. Bevels, mirrors, debris shields, hardware, and the beamline are all visible in these images and defect detection becomes more complicated. In addition, the dynamic range of these images is particularly limited. Much of the data is either near saturation, or concentrated near zero intensity, and there seems to be room for improvement in the current image acquisition techniques.

\section{Algorithm for Detecting the Optic}

Again, as with the round lens, the first step is to develop an algorithm that will isolate the optic from the rest of the image, so the defect detection can be concentrated in the area of the optic. The General Hough Transform (GHT) ${ }^{3}$ was applied for this task, since the square optic appears as a trapezoid and cannot be represented parametrically. The GHT permits the detection of any previously specified arbitrary shape, so that it can be detected in images where parts of the object are obscured or missing. Again, the GHT was implemented for the skewed square lens shape and it consistently detected the optic.

\section{Algorithm for Detecting Defects}

The more complicated background in the square lens images required a background suppression step before attempting to detect the defects. This was achieved by applying an "unsharp masking" operation. Effectively, the lower frequencies in the image are subtracted from the original image, leaving only the high frequencies. While this helps to suppress the slow-changing background, it amplifies many of the edges in the image, as well as the desired defects. Therefore, the next step is to apply algorithms that make use of morphology and texture to single out the defects. Figure 7 shows the effect of

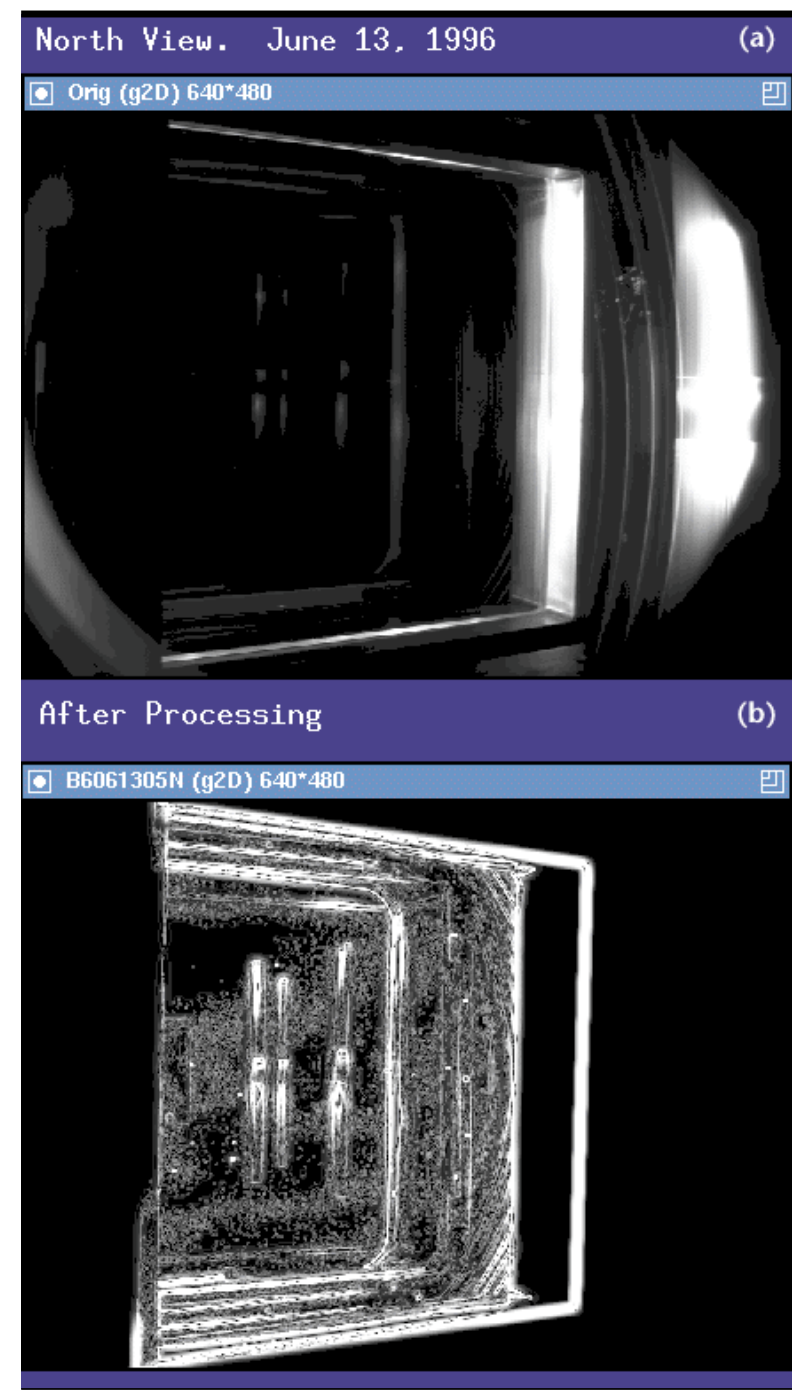

Figure 7. (a) Raw image as acquired by the Beamlet Lens Inspection System; (b) the same image data after processing. A General Hough Transform was applied to zero the surroundings of the lens. Morphological and other operators were then used to suppress the complicated background and enhance small defects and edges. The resulting enhanced image improves visualization for an observer. 
isolating the optic after applying the General Hough Transform and applying the defect enhancement algorithm. Sobel operators, top hat filters and other convolution kernels have been applied to this end. $4,5,6$ While each has its merits, none is capable of extracting just the defects. Therefore, the results of these operators should be input to a classification scheme whereby a binary decision tree could combine information from these operators to decide which pixels belong to defects and which do not. Operators that contribute information about the surroundings are just as valuable as those that contribute data about the defects, because rejecting an area is as important as classifying it as a defect. This classification step has not been implemented by this project.

As a result, automated defect detection has not been achieved for the more complicated square lens, but algorithms have been developed to isolate the optic and selectively enhance the defects so they are more readily visible to an observer as shown in Fig. 7. The defects on this lens range in size up to a maximum of $150 \mu \mathrm{m}$ according to limited manual inspection.

\section{Defect Tracking}

Once a defect is detected in any kind of lens, the defect location, area and intensity can be computed. These values are to be recorded in a database, so that the detected defects can be studied in context with prior knowledge. The database should provide the platform for documenting, addressing, and tracking defects over time.

\section{Future Work}

The NIF Project will require automated inspection of thousands of large laser optics at various stages:

1) Currently, efforts are under way to devise appropriate cleaning methods for the optics to meet contamination requirements. Cleaning methods are being applied to small pieces of optics and examined microscopically. Image analysis algorithms are needed to detect and measure remaining contamination so as to objectively evaluate the success of the various cleaning methods.

2) During assembly, transport and installation, cleaned lenses must be inspected off-line for contamination, detecting particles as small as $5 \mu \mathrm{m}$. While the off-line conditions allow dedicated image acquisition, there is a need to minimize the time for acquisition. Efforts are under way to develop an acquisition system with sufficient resolution and speed to provide adequate data for image analysis.
3) Once on-line, optics will need to be monitored to detect defects approaching the critical flaw size which may be as large as $3 \mathrm{~mm}$ on NIF. But the large numbers of optics on NIF will preclude the use of dedicated internal illumination and camera. Dark field Schlieren imaging is proposed for acquiring data from multiple optics along the beam path with one camera. Hardware and software must be developed jointly to detect defects in specific lenses by manipulating the camera's plane of focus.

4) In the meantime, studies of laser damage physics on optical materials are being conducted on a micro (nm to $100 \mu \mathrm{m})$, macro ( $\mathrm{mm}$ to $\mathrm{cm}$ ) and full-sized (m-sized) scale. Visual inspection and evaluation of these data is limited. Consistent and repeatable image processing and analysis are needed to objectively evaluate defects at these scales with high sensitivity. The results of these studies will guide future research and development leading to the actual implementation of NIF.

5) Lastly, tracking defects will require sophisticated acquisition, calibration and image analysis methods which can align and reliably relocate objects in subsequent images. The location, area and intensity information resulting from image analysis should be recorded into a dedicated database which allows the documenting, addressing, and tracking of defects over time. This database should signal an alarm when defects approach critical flaw size.

\section{References}

1. Illingworth, J ., and J. Kittler (1988), "A survey of the Hough transform," Computer Vision, Graphics and Image Processing, 44, pp. 87-116.

2. Young, I. T. (1988), "Sampling Density and Quantitative Microscopy," Analytical and Quantitative Cytology and Histology, pp. 269-275.

3. Ballard, D. H. (1981), "Generalizing the Hough transform to detect arbitrary shapes," Pattern Recognition, 13(2), pp. 111-122.

4. Laws, K. I. (1979), "Texture energy measures," Proc. Image Understanding Workshop, (Nov.), pp. 45-51.

5. Laws, K. I. (1980), "Textured image segmentation," Image Processing Institute, University of Southern California, Rept. 940, (J an.).

6. Pietikainen, M., A. Rosenfeld, and L. S. Davis (1982), "Texture classification using averages of local pattern matches," IEEE Sixth International Conference on Pattern Recognition, pp. 301-303. 


\title{
Uultistatic Micropower Impulse Radar Imaging
for Nondestructive Evaluation
}

\author{
Jeffrey E. Mast \\ Defense Sciences Engineering Division \\ Electronics Engineering
}

\author{
Brooks Johnston and Stephen G. Azevedo \\ Laser Engineering Division \\ Electronics Engineering
}

We have developed multistatic microwave imaging tools that provide an integrated platform for performing nondestructive evaluation (NDE) in a wide variety of applications. Micropower Impulse Radar (MIR) technology provides a low-cost, flexible radar that is easily adapted to multistatic modes of operation. We have expanded our MIR techniques to include bistatic and multi-bistatic imaging in both planar and circular geometries. In this report we discuss the development of these new multistatic modalities, describe the corresponding imaging systems, and show results from experiments demonstrating their performance and potential usefulness.

\section{Introduction}

Micropower Impulse Radar (MIR) technology has demonstrated its usefulness as a powerful NDE tool that is low-cost and highly portable. Successful NDEs have been performed using MIR, including the imaging of subsurface objects and the interior of civil structures. ${ }^{1-3}$ We have developed a Calibrated Autonomous Radar Testbed (CART) shown in Fig. $\mathbf{1}$, a mobile platform consisting of an MIR sensor, a linear translational stage, and a portable computer for control, acquisition and image processing. The translational stage provides antenna motion in one dimension while a computer-controlled motor drives the CART forward, providing a platform for accurate 2-D planar aperture synthesis with data suitable for 3-D imaging.

Limitations of our FY-95 MIR CART system prevented it from being used in many NDE scenarios, mainly due to geometry and apparatus constraints. The system could only handle planar geometries imaged in monostatic reflection mode, and could only use a single view of the object under inspection. Acquisition was limited to downward-looking (subsurface) acquisition, preventing other operating modes such as forward-looking or side-scanning techniques. These constraints made it very difficult to image the interior of objects or take advantage of situations in which access to more than one surface or side of objects is available. Additionally, a multistatic system capable of independent motion of the transmitter and receiver about the object under inspection provides better spatial diversity and improved visualization of interior features.

Multistatic systems consist of combinations of any of the following transmitter and receiver configurations in planar, circular, or arbitrary aperture geometries:

1) monostatic: transmitter and receiver collocated during formation of synthetic aperture;

2) bistatic: single transmitter separated from synthetically formed receiver aperture;

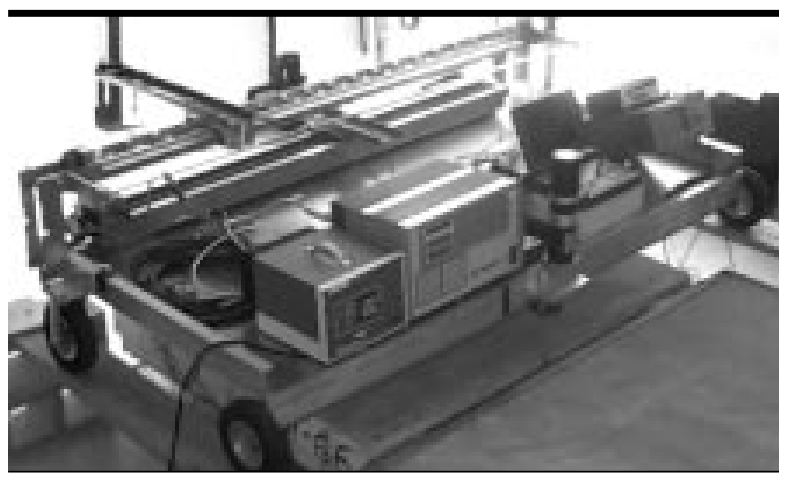

Figure 1. Calibrated Autonomous Radar Testbed (CART) for data acquisition using the MIR. 
3) multi-bistatic: multiple separated transmitters and receivers;

4) reflection mode: transmitter and receiver on same side of object; and

5) transmission mode: transmitter and receiver on different sides of the object.

There are several trade-offs in system complexity and performance for each of these implementations. Multi-bistatic systems offer a potential for improved performance over strictly monostatic or bistatic systems, but typically require longer, more complex data acquisition and more computationally intensive imaging algorithms, especially for 3-D implementations. The data acquisition rate can be improved by using arrays of elements to reduce or remove the amount of mechanical scanning required. Expansion of the MIR CART imaging system to include the hardware and apparatus capabilities to do multistatic acquisition and reconstructions greatly increases the scope and type of objects that we can nondestructively evaluate. Furthermore, a system capable of multistatic configurations is important for optimizing geometry and antenna array configurations, and determining overall imaging system parameters and performance.
Addition of these multistatic imaging capabilities not only requires development of data acquisition apparatus, but each mode also requires modified imaging techniques to accommodate the new geometrical constraints. Therefore, development and implementation of signal and image processing algorithms are required to process the data from each expanded mode of operation. These algorithms are based upon generalized methods for holographic and diffraction tomographic imaging using wideband sources. ${ }^{4-7}$ In this report we discuss our implementation of these multistatic imaging systems, as well as experiments demonstrating the performance and us efulness of these systems for microwave NDE.

\section{Progress}

In FY-96 we developed and integrated several new imaging modes described above, including multi-bistatic planar reflection mode and multibistatic circular transmission and reflection mode. This development required modifications to the FY-95 CART system to facilitate 2-D multi-bistatic linear aperture data acquisition. Other modes of operation were incorporated into the CART in the course of

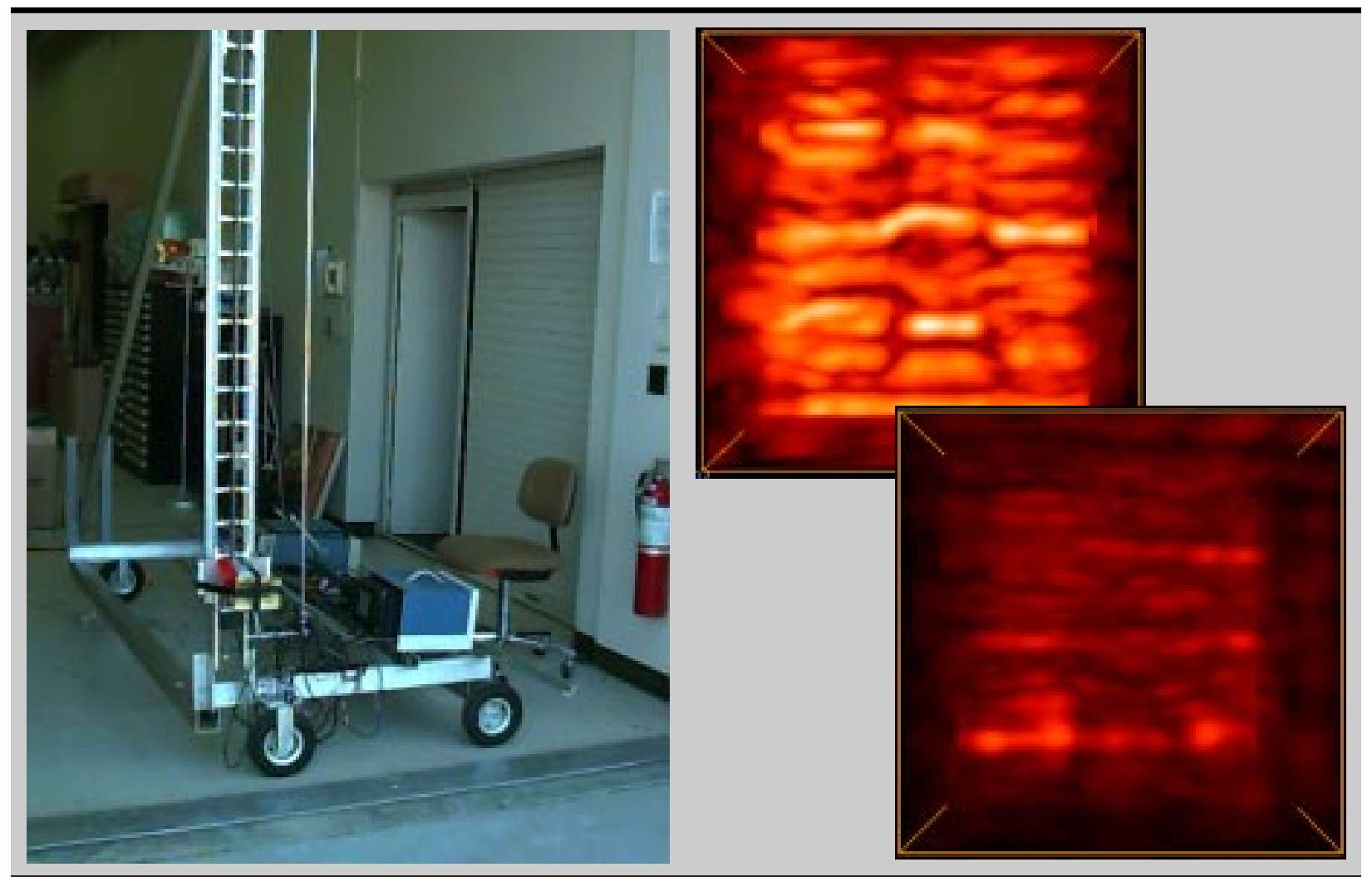

Figure 2. The CART in vertical mode, used for scanning large objects such as crates or containers, walls and other vertical objects. This mode also provides side-scanning SAR capability. The 3-D rendered images on the right are from cinder-block walls backfilled with pebbles (top) and reinforced concrete (bottom). 
making these modifications. For circular mode imaging, development of a new testbed was required. A small rotational stage with appropriate antenna mountings was developed to acquire data from all angles around an object. For each of the new modes of acquiring data, the corresponding 2-D imaging algorithms were developed, integrated and tested using the new apparatus.

\section{2-D Multi-bistatic Reflection Imaging}

The antenna mounting on the CART was modified to a boom mount, extending it in front of the chassis, giving added flexibility and reducing unwanted reflections and interference from the frame. This extension also enabled forward-looking and limited side-scanning capabilities which are useful modes for subsurface evaluations. The translational stage was hinged, allowing mobile vertical 2-D aperture formation (Fig. 2) and "unlimited" side-scanning distances. The vertical mode is useful for scanning large objects such as crates, containers, or walls. For example, the 3-D rendered images in Fig. 2 are from cinder-block walls backfilled with pebbles and reinforced concrete.

The stage was also upgraded for improved speed and positional accuracy. A second translational stage was added to facilitate independent linear motion of two antennas. Finally, the data acquisition and control software of the CART was upgraded to provide linear multi-bistatic aperture formation along the direction of the two stages.

Modular MIR 8,9 was integrated into the system and provided the flexibility required for multistatic implementations (Fig. 3). Separate transmitter and receiver modules provide bistatic capability, allowing the transmitter and receiver to be positioned independently. The operational bandwidth of this radar is approximately 1 to $6 \mathrm{GHz}$ with $10 \mathrm{MHz}$ pulse repetition frequency (PRF), a peak power of $100 \mathrm{~mW}$ and a scan rate of $40 \mathrm{~Hz}$.

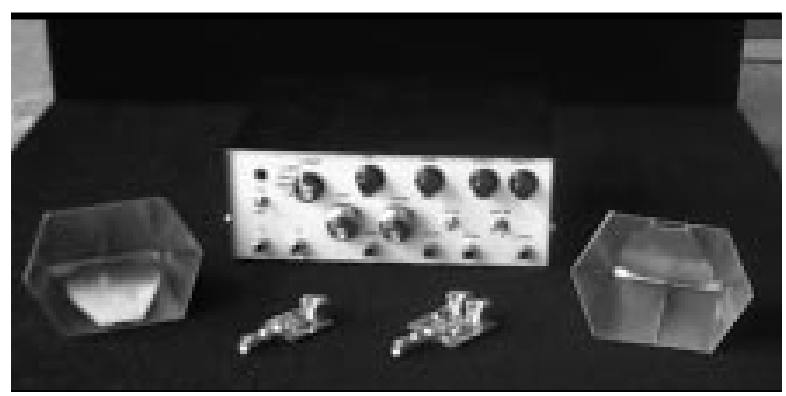

Figure 3. The modular MIR system: transmitter module, receiver module (front), two antennas (sides), and the timing and control unit (back).
We set up a target scene from which to acquire linear 2-D multistatic data consisting of a collection of metallic objects and spanning approximately $1 \mathrm{~m}$ (Figs. 4 and 5). The scene was scanned using the CART and a 1.5-m multi-bistatic synthetic aperture with $1 \mathrm{~cm}$ spacing for both transmitter and receiver. The set of monostatic equivalent data was extracted from the data set and is shown reconstructed in Fig. 5c. Also shown is a bistatic image with a single transmitter located in the center of the scene (d) along with two multi-bistatic images with combined information from multiple transmitters ((e) and (f)). The bistatic image was limited, due to the illuminating beamwidth of the radar, and therefore the entire scene is not visible. However, with as few as ten equally-spaced transmitters across the scene, the entire scene is adequately illuminated and is comparable to, if not better than the monostatic image.

To make a quantitative comparison of the different imaging modes, we must compare the clutter levels of the images. Generally, clutter is defined as anything in the image that is not a target. Two types of clutter are visible in the images of Fig. $\mathbf{5}$. The first type is side-lobe clutter resulting from the radar signal not focussed directly onto a target. The second type is multipath clutter resulting from nonlinear multiple target interactions with the radar signal not accounted for in the imaging algorithm. From the images it is clear that the multi-bistatic data has less clutter as well as better image quality for shadowed objects, such as the cylinder located beneath the four thin rods.

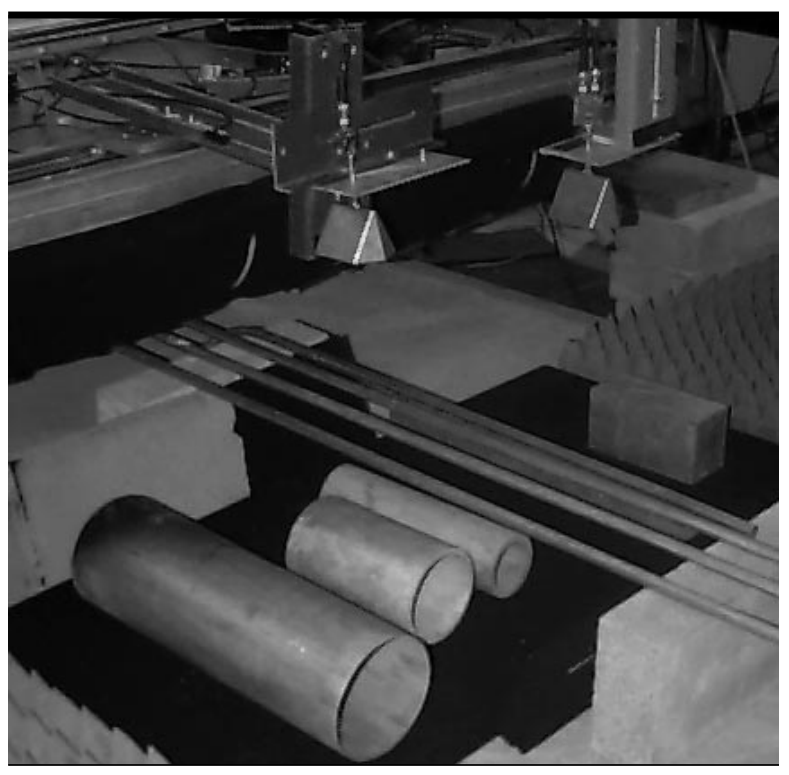

Figure 4. Target scene consisting of various metallic objects for multistatic data acquisition using modular MIR. Scanning was done horizontally across the center of the scene. Multibistatic data was acquired, consisting of 148 equally spaced transmitter locations. 
Also note that the multi-bistatic image is less susceptible to multipath clutter. A multipath artifact is visible between the right two cylinders in the monostatic image; however, in the multi-bistatic images the multipath artifacts are diminished. A quantitative measure of peak signal-to-clutter ratios (SCR) was computed for the images in Fig. $\mathbf{5}$ and is plotted in Fig. 6. Clearly only a few transmitter positions are required to achieve significant SCR improvement over the monostatic case.

The multi-bistatic images also offer improved signal-to-noise ratio (SNR) over strictly monostatic systems. This improvement results from integration of the scattered signal over all receiver positions for each transmitter used. The exact amount of improvement depends on the antenna beamwidth, because not all receivers collect energy from the scattering induced by each transmitter. This is highly dependent upon the relative position of the transmitters and receivers.

\section{2-D Circular-Mode Imaging}

A rotational stage testbed (RST) was developed to image small objects in multistatic modes (Fig. 7). Data acquisition and control software from the CART were used to acquire multistatic data from the RST. However, new imaging algorithms were developed that accounted for the circular geometry of the resulting data. Applications include small objects that can fit within the $40-\mathrm{cm}$ diameter of the stage, or more specifically, cylindrical objects such as utility poles and small concrete columns. We chose a portion of a utility pole for initial testing and evaluation. In Fig. 7, a section of a utility pole is imaged on the rotational stage using a monostatic configuration. Data was acquired from a single rotation of the utility pole while illuminating it with a stationary monostatic MIR configuration, obtaining 437

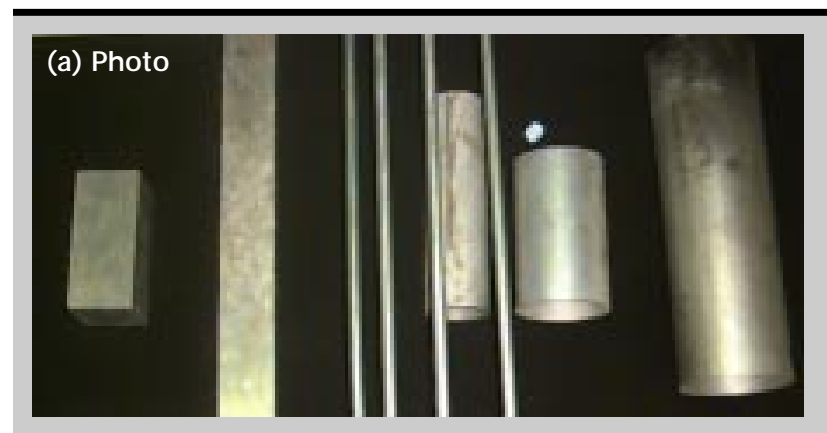

(b) Schematic of cross-section imaged
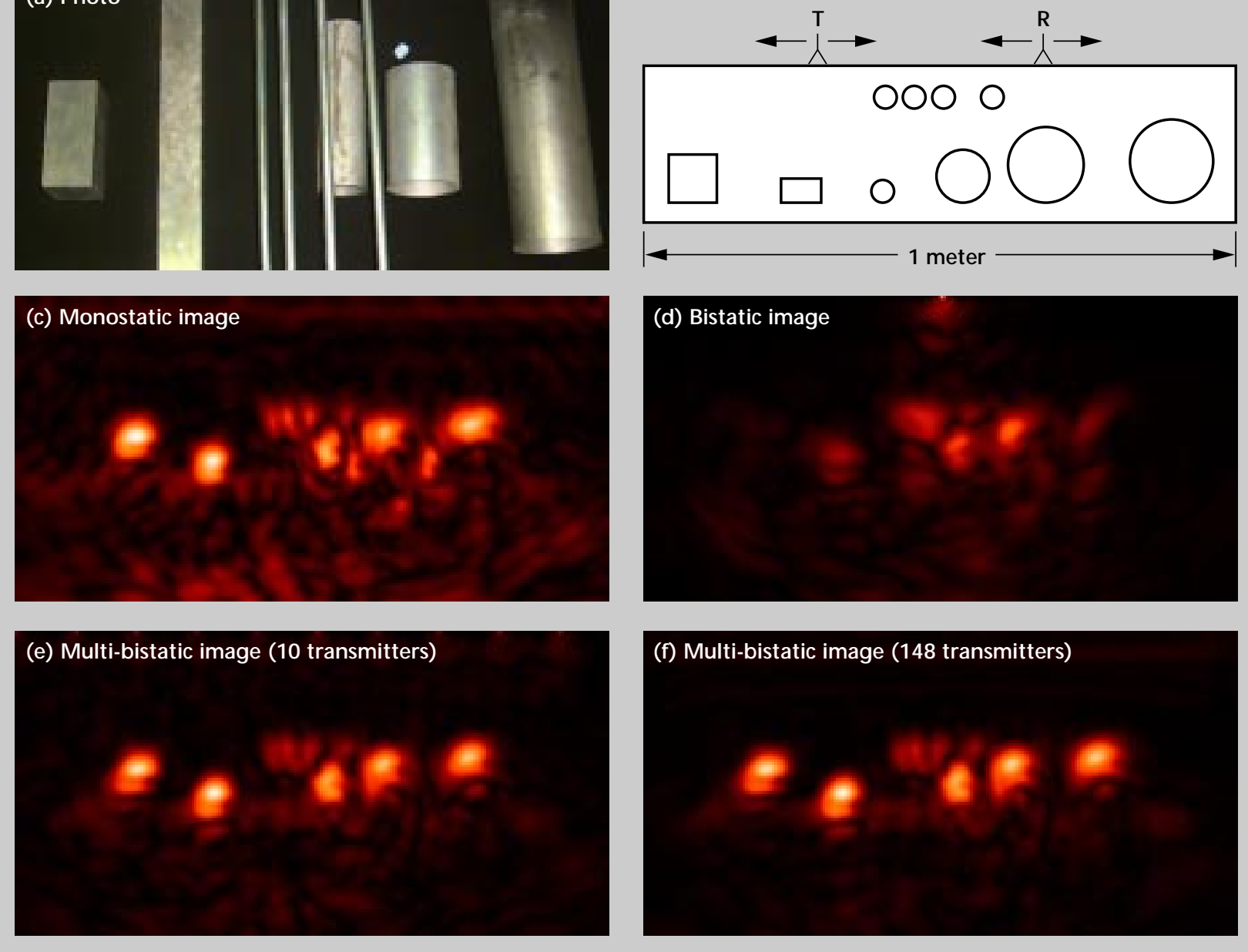

Figure 5. Images of the target scene using different imaging modes. Each image was obtained using 148 receiver positions separated by $1 \mathrm{~cm}$. The monostatic data was acquired from a single $T / R$ forming a synthetic aperture. For the bistatic image, the transmitter was positioned directly over the center of the scene with the receivers spanning the entire aperture. For the multi-bistatic cases, the transmitters were equally spaced across the scene. 
equally spaced samples around the circular aperture. This technique combined with subsequent image processing easily images the $2-\mathrm{cm}$-wide fissure within the utility pole.

We also performed multi-bistatic data acquisition from the utility pole. A transmitter was attached to the stage such that it rotated with the object while an MIR receiver was held stationary. Data was then acquired from a single rotation of the pole and the process was repeated for each of 36 equally spaced transmitter locations. The multi-bistatic technique, with only a single $T / R$ pair, is significantly more time-consuming than the monostatic data acquisition, because a full rotation of the stage must be done for each transmitter location. However, similar to the linear multistatic method, it provides better SCR than the monostatic scenario, as well as

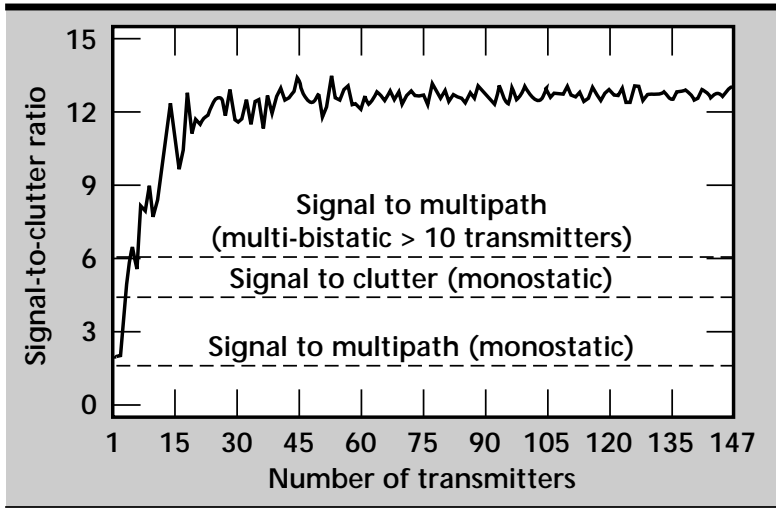

Figure 6. Signal-to-clutter ratio for multi-bistatic image. Dashed lines represent signal-to-clutter and signal-tomultipath levels for the multi-bistatic and monostatic images. Multipath artifacts represent multiple bounces of the radar signal which are nonlinearities unaccounted for by the imaging algorithm.

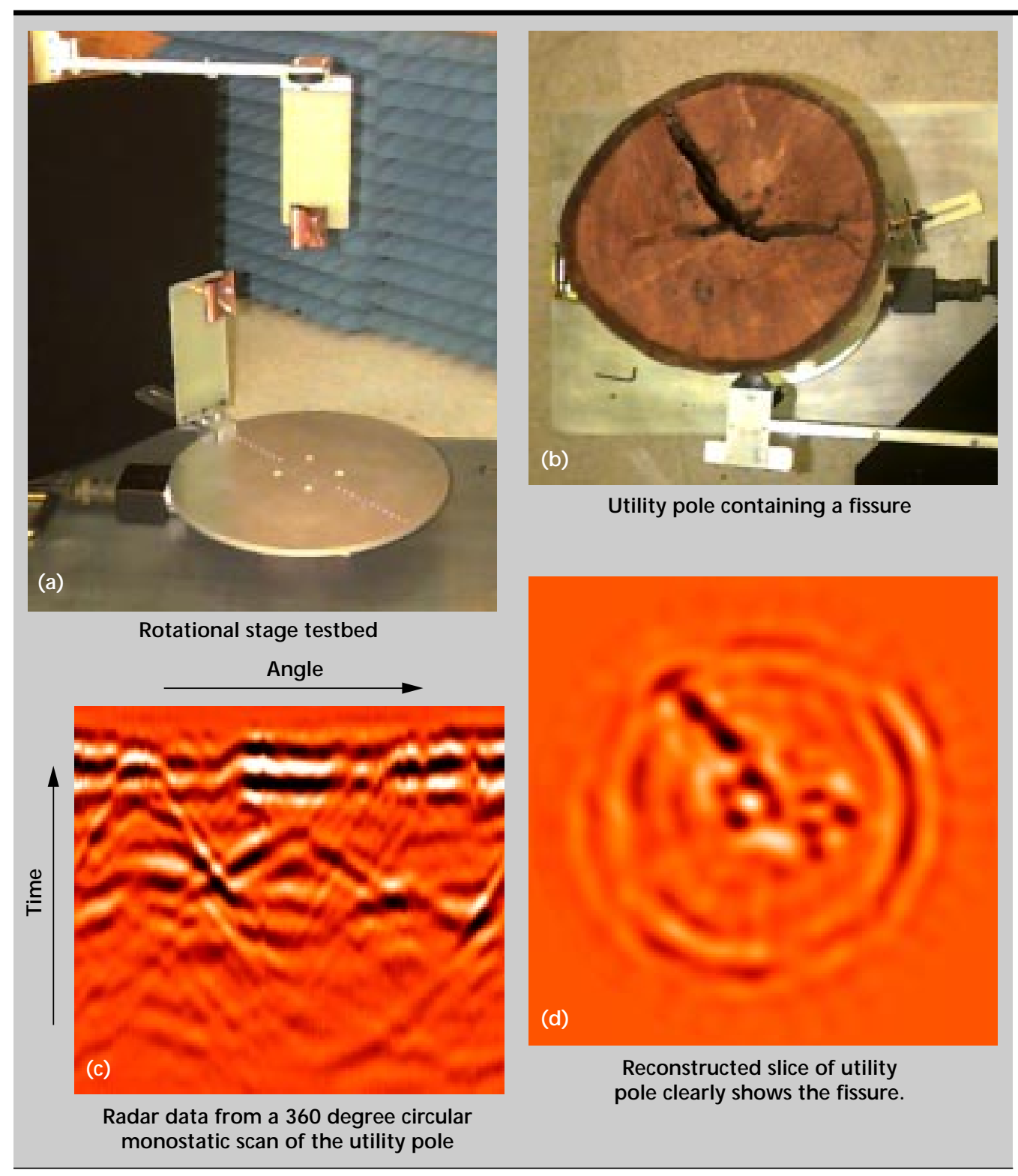

Figure 7. Rotational stage used to acquire monostatic circular reflection profile data from a utility pole (a). Impulse radar data was acquired from 437 equally-spaced sample points around the circumference of the pole (b). The data (c) was reconstructed to produce an image of the 2-cm fissure in the pole (d). 
better visualization of some of the salient features of the fissure. This improvement is clear in the multibistatic reconstructions of the fissure shown in Fig. 8.

In the full reconstruction, 36 bistatic views are combined to image the features within the utility pole, but 9 views are sufficient to reconstruct the image without significant degradation. In general, the required number of transmitters will vary, depending on the size of the object and the beamwidth of the antenna.

\section{Future Work}

Our future work involves concentration in three areas:

1) improving radar characteristics, including improved antennas, higher frequencies, and higher PRF;

2) modifying data acquisition to involve antenna arrays and custom apparatus for hand-held and other specialized applications; and

3) expanding imaging capabilities to include 3-D multistatic and circular geometries as well as accounting for object shape and other applicationspecific signal processing considerations.

In this report we discussed non-coupled data acquisition in which there was an air gap between the antenna and the object. Improved SNR and resolution can result from matching the antenna characteristics more closely to the dielectric properties of the material under inspection. This matching requires dielectrically-loaded antennas that couple the radar directly to the material. The resulting antenna would be more compact and would induce less ringing between the surface of the object and the antenna than the air-launching antennas. Furthermore, optimal frequency selection for the radar could result in improved performance.
The exact frequency band is highly applicationdependent, due to the wide variety of attenuation characteristics of different media types. For the utility pole application, increasing the frequency to $10 \mathrm{GHz}$ could improve resolution, detection, and visualization of interior features such as cracks, rot, and water damage. Further testing is required to determine the optimal radar frequency band for inspection of utility poles.

Data acquisition times for multistatic scenarios can be quite large, especially when taking data using only a single T/R pair. Future work would incorporate antenna arrays or other techniques which could reduce acquisition times significantly. With multiple element systems, mutual coupling between antenna elements and optimization of array performance will have to be considered. The specific implementation details will be dependent on the given application. However, in general, increasing the radar effective PRF and using multiple T/R pairs will provide increased acquisition rates.

The technique we used for imaging the utility pole did not account for the non-circular shape of the pole. Similarly, the planar imaging method did not compensate for non-planar surfaces. If not compensated for, these nonlinearities cause phase distortion, resulting in image degradation. Future work involves accounting for these nonlinearities, at least to first order, to help improve resolution and visualization of interior object features.

\section{Acknowledgments}

We would like to thank T. McEwan, G. Dallum, and P. Welsh for their work on the MIR radar system development; M. Vigars and R. Greenwood for their support in the development of the RST and modifications to the CART; G. Governo, M. Fillipucci, and T. Ferriera for their support in the development of

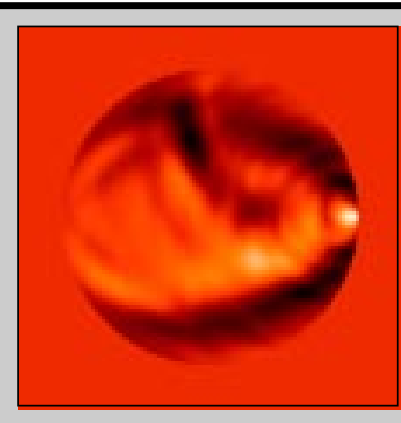

1 transmitter

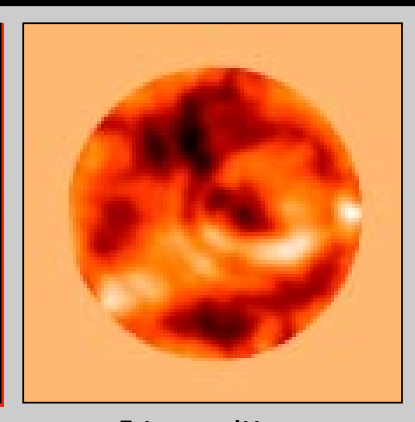

5 transmitters

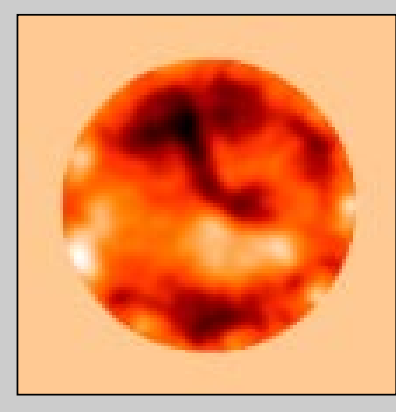

9 transmitters

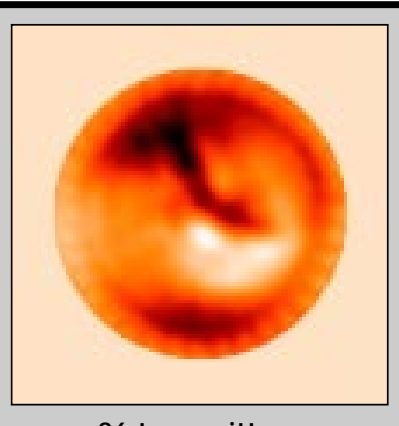

36 transmitters

Figure 8. Multi-bistatic reconstructions using indicated number of equally-positioned transmitters around the utility pole. In all cases, 437 receiver positions were used. Note that the multi-bistatic image has better signal-to-clutter than the monostatic image of Fig. 7. 
the CART; T. Rosenbury and D. Mullenhoff for their work in designing and building antennas; $\mathrm{S}$. Nelson for his input and discussion concerning system design, implementation and results; $H$. J ones and $M$. Wieting for their assistance with the data acquisition and control hardware and software; and $M$. Newman for his assistance with the controller hardware. Finally, we would like to thank H. Martz for his support of this work.

\section{References}

1. Mast, J. E., and S. G. Azevedo (1996), Applications of Micropower Impulse Radar to Nondestructive Evaluation, Lawrence Livermore National Laboratory, Livermore, Calif. (UCRL 53868-95).

2. J ohansson, E. M., and J. E. Mast (1995), Ultra-wideband radar imaging for the nondestructive evaluation of bridges, Lawrence Livermore National Laboratory, Livermore, Calif. (UCRL 53868-94).

3. J ohansson, E. M., and J. E. Mast (1994), Imaging algorithms for synthetic aperture ultra-wideband radar, Lawrence Livermore National Laboratory, Livermore, Calif. (UCRL 53868-93).
4. Devaney, A. J. (1982), "A filtered backpropagation algorithm for diffraction tomography," Ultrasonic Imaging, Vol. 4, pp. 336-350.

5. Porter, R. P., and A. J . Devaney (1982), "Generalized holography and computational solutions to inverse source problems," J ournal of the Optical Society of America, Vol. 72, pp. 1707-1713.

6. Porter, R. P. (1989), "Generalized holography with application to inverse scattering and inverse source problems," Progress in Optics XXVII, E. Wolf, Ed., Elsevier Science Publishers B.V., pp. 317-397.

7. Chew, W. C. (1990), Waves and Fields in Inhomogeneous Media, Van Nostrand Reinhold, New York, N.Y.

8. Azevedo, S. G., and T. E. McEwan (1997), Millimeterwave MIR, Lawrence Livermore National Laboratory, Livermore, Calif. (UCRL 53868-96).

9. Azevedo, S. G., T. E. McEwan, and J. P. Warhus (1996), Microradar Development, Lawrence Livermore National Laboratory, Livermore, Calif. (UCRL 53868-95). 



\title{
ulti-modal NDE for AVLIS Pod Shielding
Components
}

\author{
Diane J. Chinn, Nancy K. Del Grande, and Dwight E. Perkins \\ Manufacturing and Materials Engineering Division \\ Mechanical Engineering
}

We are assessing several non-destructive evaluation (NDE) techniques as possible replacements for existing pod component inspection methods for the Advanced Vapor Laser Isotope Separation (AVLIS) program at Lawrence Livermore National Laboratory (LLNL). The present inspection method uses dye-penetrant testing to inspect surfaces of components for cracks. Because this type of testing is labor-intensive, subjective, and evaluates only the surface of components, five other methods are under consideration. Each technique in our assessment, digital radiography, ultrasonic testing, optical imaging, dual-band infrared imaging and dye-penetrant testing, is evaluated for performance criteria that will be important to production runs.

\section{Introduction}

The AVLIS program at LLNL is presently designing a pilot demonstration facility. When demonstration runs begin, high through-put will require all aspects of the production process to be streamlined and robust. Shielding components in the AVLIS pod undergo mechanical and thermal loading during use. Components with stress-induced cracking caused by these loads must be replaced upon detection of cracks.

During the conceptual design phase, visual dyepenetrant testing was used to inspect the shielding components for cracks. Although dye-penetrant testing is especially labor-intensive, the low throughput in the conceptual design phase allowed designers to use the technique because it minimized capital costs. Other NDE techniques may be more suitable for large volume inspection. ${ }^{1}$

We are currently assessing several techniques for NDE of pod components for the AVLIS pilot facility design as possible replacements for existing pod component inspection methods.

AVLIS designers are seeking faster inspection methods that are reliable, recordable, capable of finding internal cracks and detecting cracks occurring on inaccessible surfaces. The present dyepenetrant inspection method takes as long as 5 to 6 days for a complete evaluation of all the pod components. Because it is a surface inspection method, dye-penetrant testing cannot find internal cracks, nor can it find cracks on the inner diameter of tubular sections.

The present dye-penetrant technique does not include digital archiving of the results. Digital archiving is important for production runs because components with cracks occurring at non-critical locations are often not replaced. Archiving of the inspection results allows inspectors to track any crack growth which may occur in non-critical locations.

We are evaluating five different NDE techniques for detecting cracks in shielding components of the AVLIS pod. Each technique, digital radiography, ultrasonic testing, optical imaging, infrared imaging and dyepenetrant testing, is evaluated for (1) ability to find surface-breaking and internal cracks as small as $1.6 \mathrm{~mm}$ in length; (2) ability to inspect the inner diameter of tubular components; (3) potential for automation; (4) digitization of inspection results; (5) reliability and accuracy in correctly distinguishing cracks from other defects; (6) ability to be handled in a glove box; and (7) rapid evaluation of complex shapes.

\section{Progress}

The pilot facility will contain approximately 120 pod shielding components. The three major component shapes are plates, tubes and troughs. A few odd-shaped components will also need to be 
inspected. We tested several components, of which one plate contained a crack. Because of the stress profile on the components, cracks almost always propagate through the thickness, rather than circumferentially or in a plane.

Shielding components are contaminated during normal AVLIS operations and must be inspected in a glove box with appropriate health and safety precautions. Some of the inspection methods are appropriate only for certain shapes, while other methods would be difficult to use in a glove box.

So far, we have had success detecting cracks and material variations with ultrasonic testing, infrared imaging and dye-penetrant testing of several components. Digital radiography and optical imaging do not appear suitable for pod components.

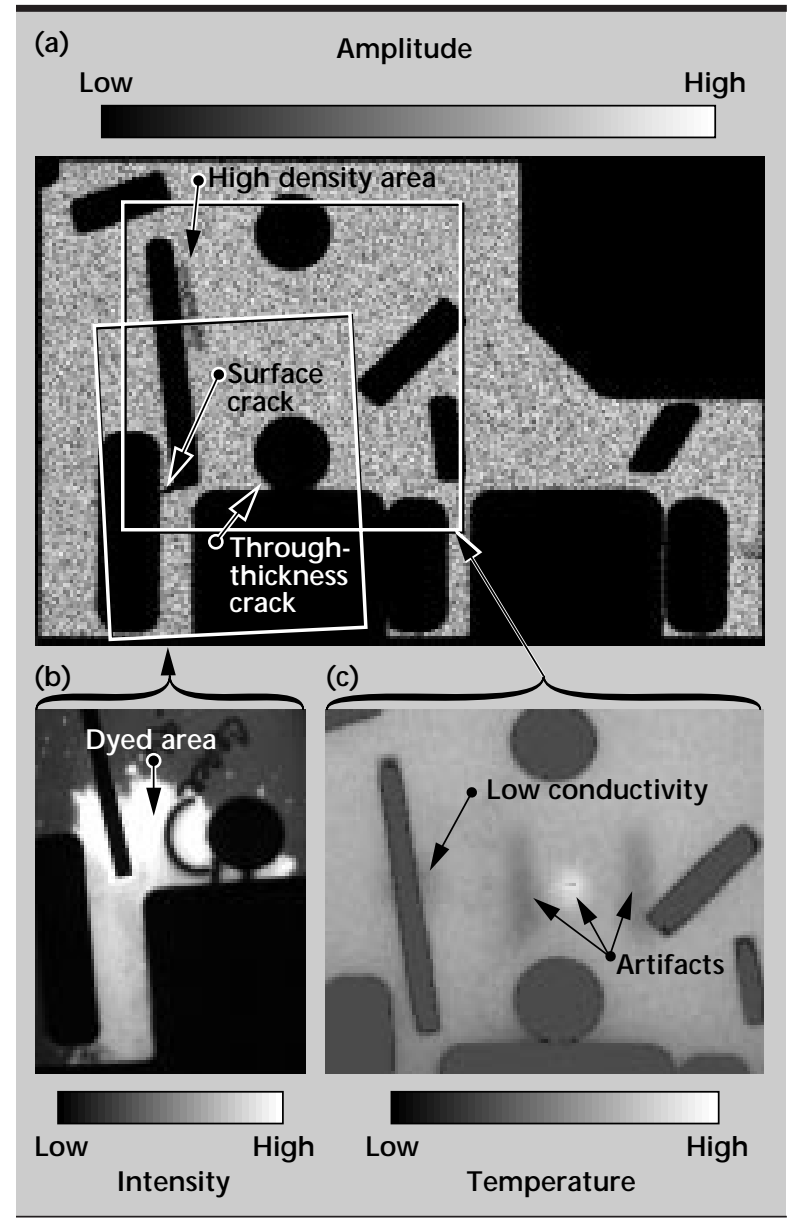

Figure 1. Imaging of AVLIS pod components using three techniques. (a) Ultrasonic imaging of the plate component shows cracks and density changes. Areas included in (b) and (c) are shown on the ultrasonic image. (b) Optical imaging of dyepenetrant testing can be used to archive results of the inspection. The arrow shows the area where dye penetrant has been applied. (c) Infrared imaging shows the progression of heat conduction through the part. Artifacts in the image result from the collection system.

\section{Ultrasonic Testing}

Ultrasonic inspection uses acoustic waves traveling through the specimen to interrogate the surface and interior of the specimen for defects. Changes in homogeneity of the material alter the characteristics of the ultrasonic wave. By scanning the specimen, we obtain an ultrasonic image of the specimens showing variations in homogeneity.

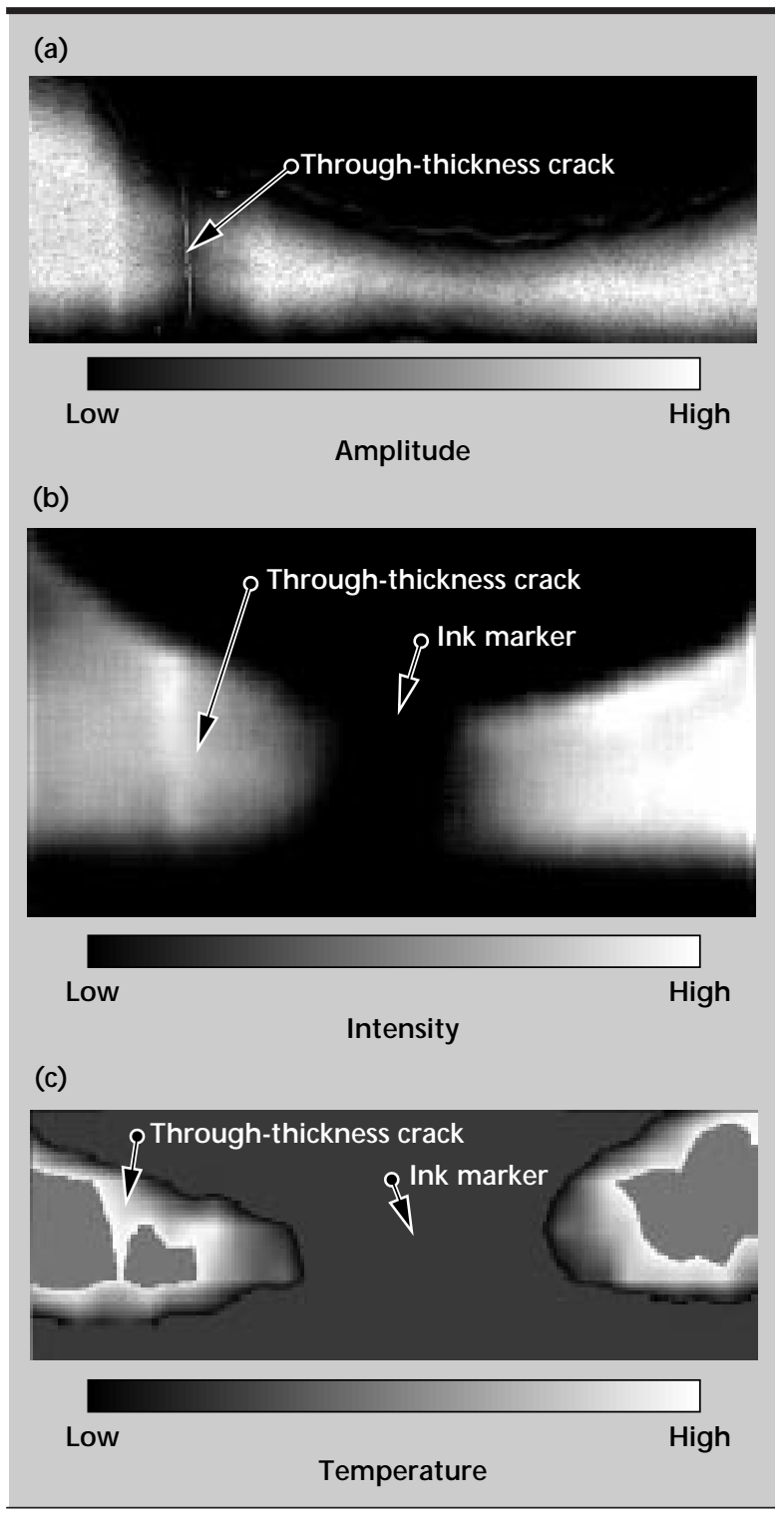

Figure 2. A through-thickness crack imaged using the three inspection techniques. (a) Ultrasonic imaging of surface wave propagation shows that very little energy propagates across a through-thickness crack. (b) Optical imaging of dye-penetrant testing records the location of the crack. (c) Infrared imaging shows discontinuity in thermal transfer across the throughthickness crack. The dark line to the right of the crack is from pen markings. 
Figure 1a shows an ultrasonic image of a plate component. Areas with density variation, a throughthickness crack and a surface crack are indicated in the image. Figures $\mathbf{2 a}$ and $\mathbf{3 a}$ show high-resolution ultrasonic images of the through-thickness crack and the surface crack, respectively. The images in Fig. $\mathbf{2 a}$ and $\mathbf{3 a}$ plot the amplitude of a surface wave traveling along the surface of the plate. The surface wave has very low amplitude traveling across a through-thickness crack (Fig. 2a) and slightly decreased amplitude traveling across a surface crack (Fig. 3a) depending upon the depth of the crack. These images demonstrate the capabilities of ultrasonic testing in finding and characterizing defects.

The disadvantages of using ultrasonic testing include the need for a couplant such as water to introduce acoustic waves into the material. Different scanning configurations are required for each shape making the ultrasonic technique expensive if multiple scan systems are required for each shape. In addition, high-resolution scanning generally takes 15 to $30 \mathrm{~min}$ to scan specimens of the size of pod components.

\section{Dye-penetrant Testing}

This method is presently used to manually inspect the pod components. A fluorescent dye is painted onto the surface of the component and is absorbed by surface-breaking cracks. Filled with dye, these cracks are easily detected by visual inspection of the part under ultraviolet light. Many different shapes are easily inspected with this method.
Internal defects can not be detected with dyepenetrant testing. The disadvantage of this technique is that it is time-consuming and difficult to implement when inspecting the inner diameter of tubular sections. This technique can be archived easily using an optical recording system. An optical image of the pod component, through-thickness crack and surface crack filled with dye are shown in Figs. $\mathbf{1 b}, \mathbf{2} \mathbf{b}$, and $\mathbf{3 b}$, respectively.

\section{Infrared Imaging}

Infrared imaging uses the conductivity of heat through the specimen to evaluate the surface and interior of the specimen. After flashing the material with a few milliseconds of intense uniform light, variations in thermal conductivity of the part are revealed by imaging the surface with an infrared camera. $^{2}$ One feature of infrared imaging is that it can inspect large areas of specimens with complex shapes very quickly. Figures $\mathbf{1 c}, \mathbf{2 c}$, and $\mathbf{3 c}$ show infrared images of the pod component.

\section{Radiographic Testing}

Radiography uses the transmission of $x$ rays through a material to image differences in density. Cracks are generally imaged best with the source transmitting parallel to the plane of the crack. Even under ideal source-crack configurations, cracks must still have sufficient size in order to be resolved by film or digital radiography. For this reason, we found that radiographic (a)

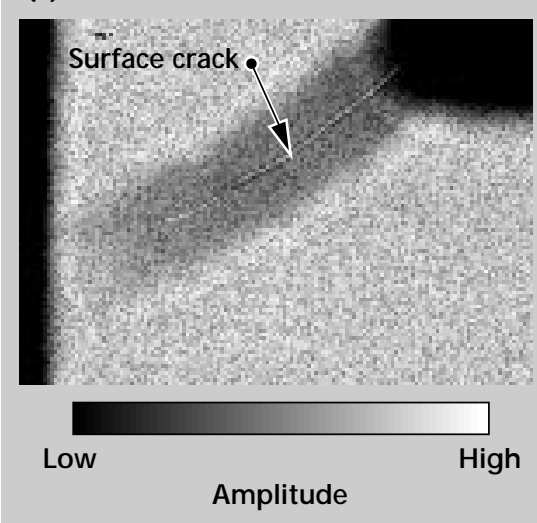

(b)

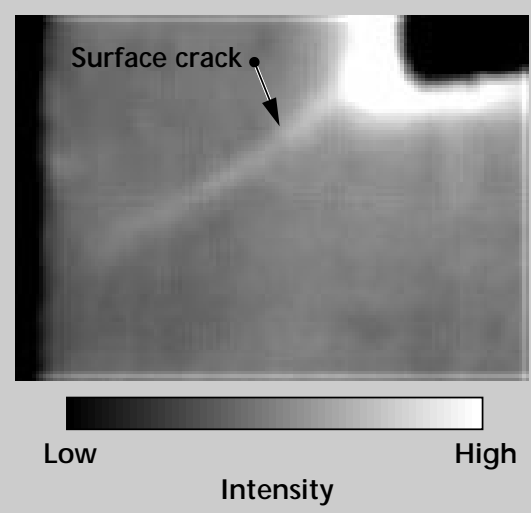

(c)

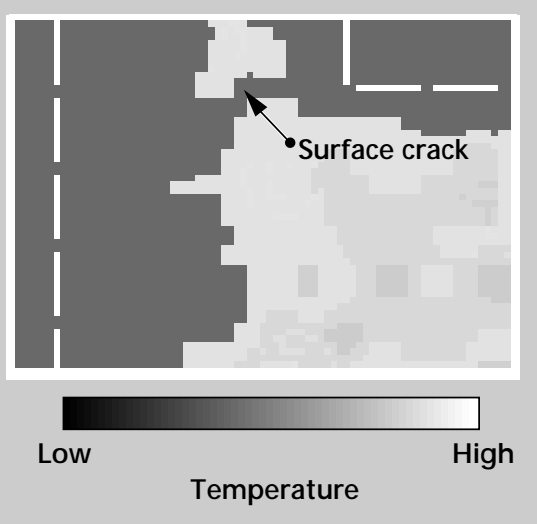

Figure 3. Imaging of a surface crack by the three inspection techniques under consideration. The arrow shows the location of the surface crack indication in each image. (a) Ultrasonic image of surface wave propagation shows partial attenuation of the wave as it passes over the surface crack, transmitting some of its energy. (b) Dye-penetrant testing easily detects surface cracks. (c) Infrared imaging indicates a decrease in the thermal conductivity at the location of the surface crack. Dotted lines show the edges of the test specimen near the crack. 
Table 1. Evaluation of inspection techniques. Each technique is evaluated according to its ability to satisfy the performance criteria.

\begin{tabular}{lccccc}
\hline $\begin{array}{l}\text { Performance } \\
\text { criteria }\end{array}$ & $\begin{array}{c}\text { Ultrasonic } \\
\text { testing }\end{array}$ & $\begin{array}{c}\text { Infrared } \\
\text { imaging }\end{array}$ & $\begin{array}{c}\text { Dye-penetrant } \\
\text { imaging }\end{array}$ & $\begin{array}{c}\text { Radiographic } \\
\text { testing }\end{array}$ & $\begin{array}{c}\text { Optical } \\
\text { imaging }\end{array}$ \\
\hline Surface cracks & $\sqrt{ }$ & $\sqrt{ }$ & $\sqrt{ }$ & $\times$ & $\times$ \\
Internal cracks & $\sqrt{ }$ & $\sqrt{ }$ & $\times$ & $\times$ & $\times$ \\
Inside tubes & $\sqrt{ }$ & $\sqrt{ }$ & $\sqrt{ }$ & $\times$ & $\sqrt{ }$ \\
Automation & $\sqrt{ }$ & $\sqrt{ }$ & $\sqrt{ }$ & $\sqrt{ }$ & $\sqrt{ }$ \\
Digital archiving & $\sqrt{ }$ & $?$ & $\sqrt{ }$ & $\times$ & $\times$ \\
Defect classification & $\times$ & $\sqrt{ }$ & $\sqrt{ }$ & $\sqrt{ }$ & $\sqrt{ }$ \\
Complex shapes & $\sqrt{ }$ & $\sqrt{ }$ & $\sqrt{ }$ & \\
Glove box inspection & & & 5 & 3 & 3 \\
Inspection time & 15 & 2 & & & \\
(min), 1 plate & & & & & \\
\hline
\end{tabular}

$\sqrt{ }$ - satisfies criteria $\quad \times$ - unacceptable or not possible

? - to be determined

testing is not suited to this application because cracks in the pod components are not large enough to be detected.

\section{Optical Imaging}

Optical imaging records the transmission of light through the part to detect inhomogeneities. As with radiographic testing, optical transmission requires that a crack be large enough relative to the detector sensitivity in order to be detected. We found that optical imaging is not suitable for pod components because of the small crack sizes.

\section{Preliminary Assessment}

Ultrasonic testing, dye-penetrant testing and infrared imaging show promise as pod component inspection methods. We have begun to assess these three techniques for different component shapes based on AVLIS inspection needs. So far, we have performed ultrasonic testing on plate and tubular members, infrared imaging on plate and trough components, and optical imaging of dye-penetrant testing on plates. The matrix in Table 1 summarizes our preliminary findings on each technique for AVLIS inspection performance criteria.

Radiography and optical imaging have been eliminated from consideration because they cannot find surface or internal cracks easily. Of the remaining techniques, ultrasonic testing takes the longest time to inspect a plate component. Infrared imaging has the fastest inspection time, however, we are still working on interpretation of the images for defect classification.

\section{Future Work}

Comprehensive NDE of AVLIS pod components will most likely involve several modes of inspection. We have begun to collect images, perform image analysis and processing on each of the remaining three NDE methods. We will continue our work on other shapes and other types of defects. This work provides guidance for the selection of improved inspection methods that will significantly impact the operations of the AVLIS system.

\section{References}

1. Chinn, D. J., D. J. Schneberk, N. K. Del Grande, and G. H. Thomas (1996), "A comparison of quantitative imaging techniques for lightweight materials," J. Acoust. Soc. Am., 100 (4), October.

2. Del Grande, N. K., P. F. Durbin, and D. E. Perkins (1996), "Infrared computed tomography for characterizing structural defects," Engineering Research Development and Technology Thrust Area Report, Lawrence Livermore National Laboratory, Livermore, Calif., (UCRL 53868-95). 

